\title{
CHARACTERISTICS AND INTERPRETATION OF THE PHOTOMETRIC VARIABILITY OF ETA CARINAE AND ITS NEBULA*
}

\author{
A. M. VAN GENDEREN \\ Leiden Observatory, Postbus 9513, 2300 RA Leiden, The Netherlands \\ and \\ P. S. THE \\ Astronomical Institute 'Anton Pannekoek', University of Amsterdam, Roetersstraat 15, 1018 WB \\ Amsterdam, The Netherlands
}

(Received 19 November, 1984)

\begin{abstract}
A short review is given on the history of the peculiar variable object $\eta$ Car and on a number of relevant references describing and discussing its physical characteristics and behaviour, based on different types of observational techniques. The star is known to be variable since the 17 th century. The excessive mass loss to which it was subject during the 19th century is now visible as an ellipsoidal reflection nebula of 15" diameter: the so-called 'homunculus'. The remainder of the paper is spent on different kinds of problems partly based on the results of a decade of photometric monitoring in the VBLUW photometric system of Walraven. Foreground reddening and reddening by dust in the homunculus are determined and amount to $E(B-V)_{\mathrm{J}}=0^{m} \cdot 50$ and $<0^{m} \cdot 6$, respectively. Scanning of the homunculus revealed an estimate for the photometric characteristics of the central object, which presumably consists of a massive hot star surrounded by a cooler gas envelope. The total luminosity is derived using fluxes of various sources in the wavelength region $0.15<\lambda<175 \mu \mathrm{m}$ resulting in $M_{\mathrm{bol}}=-12^{m} \cdot 3 \pm 0^{m} \cdot 2$. The total observed flux corrected for foreground extinction corresponds to a star with $R \sim 96 R_{\odot}$ if $T_{\text {eff }} \sim 30000 \mathrm{~K}$. The mass may be near $150 M_{\odot}$. The excess luminosity in 1843 , when the star was presumably bolometrically at least $\sim 2^{m} .5$ brighter than at present, may have been caused by envelope-energized pulsations when the star's luminosity was close to its Eddington limit. The temperature should then have been $\sim 50000 \mathrm{~K}$. The mass loss rate, during the excess luminosity phase lasting $\sim 30 \mathrm{yr}$, is estimated to amount to $\dot{M} \sim 4 \times 10^{-3} M_{\odot} \mathrm{yr}^{-1}$. At present the mass loss may be $\dot{M} \sim 10^{-4} M_{\odot} \mathrm{yr}^{-1}$. Since the homunculus is mainly built up from material expelled in the $30 \mathrm{yr}$ interval (from $\sim 1830$ to 1860 ), its total mass amounts to $M_{\text {hom }} \sim 0.15 M_{\odot}$. The historical observations of the colours of $\eta$ Car and a comparison with the characteristics of S Dor type stars, suggest that $\eta$ Car was subject to a number of S Dor type phases similar to those of P Cyg (in the 17th century), $\mathrm{S}$ Dor and others. A satisfactory explanation is found for the complete historical light curve. The decrease in light after the 1843 maximum by $\sim 9^{m}$, presumably consists of a fading of the luminosity excess and the S Dor effect by $\sim 2^{m} .5$ and $\sim 3^{m}$, respectively, and a $\sim 3^{m} .5$ extinction by circumstellar dust. The small amplitude light variations which $\eta$ Car showed during the last decade, were studied with the aid of the variations of the Balmer jump. They are presumably caused by temperature variations of the central star.
\end{abstract}

\section{Introduction}

A great number of papers have been published on one of the most enigmatic objects in the Milky Way: the variable star $\eta$ Car or in the old astronomical literature $\eta$ Argus (coordinates are given in Figure 1). The fact that $\eta$ Car is a member of a young open cluster $\operatorname{Tr} 16$ (identification charts given by Feinstein et al., 1973; Thé et al., 1980a) in

* Based partly on observations collected at the ESO, La Silla, Chile.

Space Science Reviews 39 (1984) 317-373. 0038-6308/84.15.

○ 1984 by D. Reidel Publishing Company.

(C) Kluwer Academic Publishers - Provided by the NASA Astrophysics Data System 
3847. $\eta$ ARGÛ́S.



Recent discoveries by Sir David Gill and Professor E. C. Pickering shew that the photographic spectrum of $\eta$ Argus resembles that of the "New Stars." It seemed therefore desirable to arrange in one table the curious light changes of this star. It has been possible to do this with some amount of success. The history of this star is divided into two stages - the first, when the star was not suspected of variation. This period stretches from the time of Ptolemy to the observations of Burchell who seems to have been the first to find any irregularity in the star's light. 'The contemporaneous meridian observers of that epoch, however, do not seem to have noticed this variation and they probably, as Rumker openly avows he did, used Lacaille's magnitude without thought of change. It is a former director of this observatory (Henderson) who shews that had the star been bright in the time of Ptolemy, that it would, no doubt, have been observed by him, as the chief stars of the Southern Cross, Centaurus, etc., which culminate at Alexandria at about the same altitude as $\eta$ Argâs, are to be found in the Almagest. As the next recorded observation (by Halley in 1677) is of the 4th magnitude, it is perhaps a fair inference to suppose that at the time of Ptolemy, the star was even fainter. There may thus be a certain amount of continuity in the increase of this star's light for many centuries, but of course this is all most uncertain.

Fig. 1. The beginning of Innes' (1903) paper on the historical light variation of $\eta$ Car (then still called $\eta$ Argus).

J. Herschel's: 'Key Hole Nebula', NGC 3372 or Carina Nebula, is taken as an indication that the star is a very young object. Figure 2 shows the original drawing (as a negative) of the central part of the nebula by Herschel made at the Cape of Good Hope

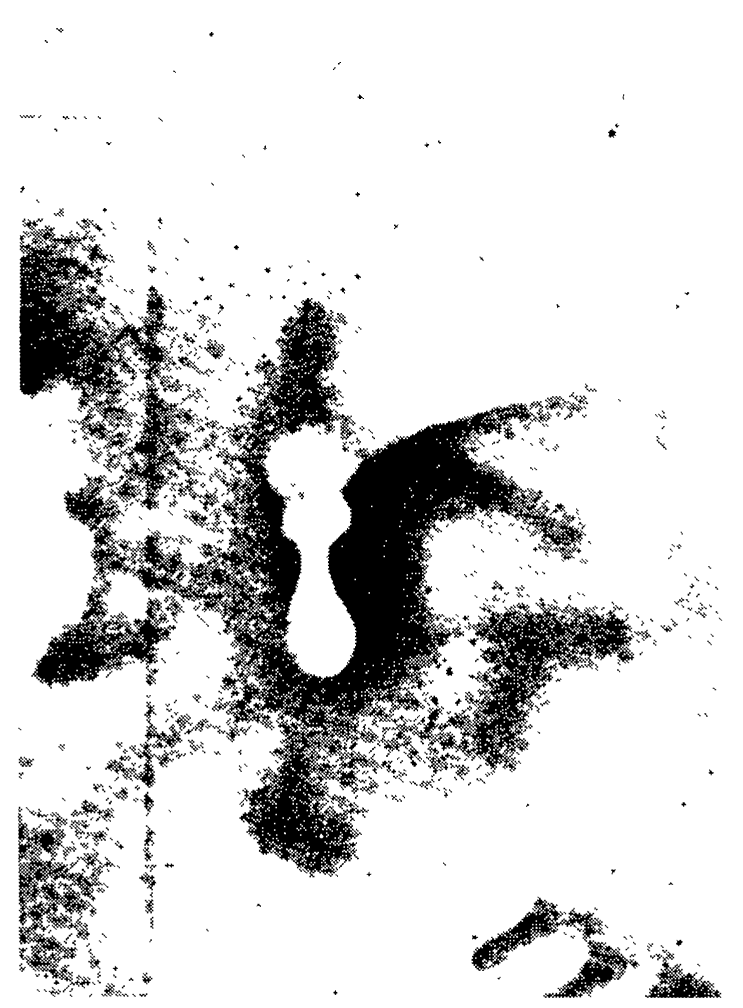

Fig. 2. Copy of the original drawing (as a negative) of J. Herschel's 'Key Hole Nebula' in the great Carina Nebula, made around 1835. Eta Car is situated at the right of the key hole in the heavy shaded area ( $N$ is at the top, E to the right). The total length of the key hole is $\sim 10^{\prime}$. Its shape is now slightly different. 
around 1835. Eta Car is situated to the right of the Keyhole in the heavy shaded area. Figure 3 is a recent photograph of the Carina Nebula in red light.

Eta Car underwent a series of outbursts in the 17th to the 19th century, in which the

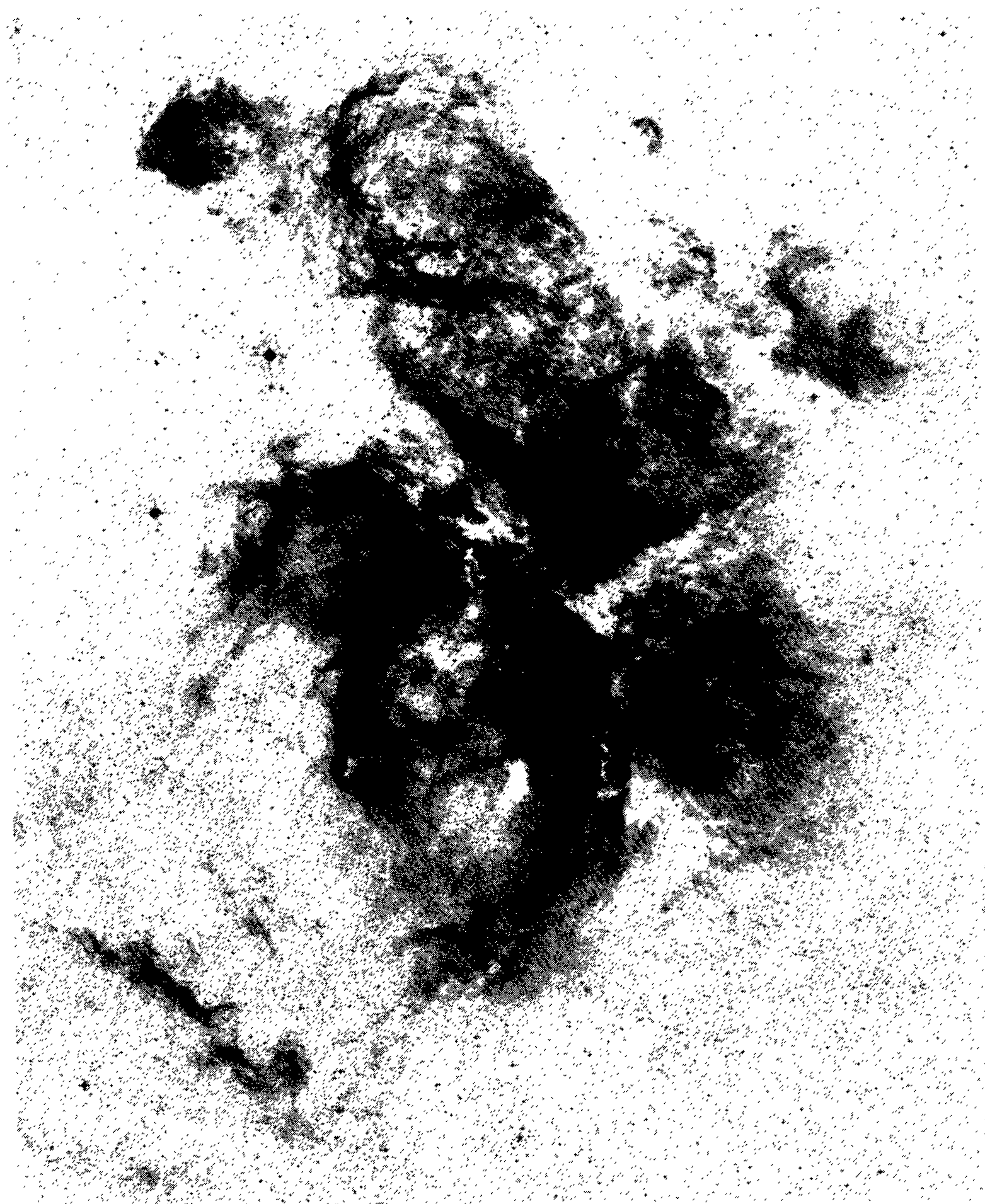

Fig. 3. Negative of the Carina Nebula or NGC 3372 in red light. The keyhole is situated in the middle of the black triangle-shaped top part of the nebula, but because of over exposure not well visible. $\mathrm{N}$ is at the top, E to the right (UK Schmidt Southern/SR Atlas). 
visual magnitude varied from 2 to -1 (Innes, 1903; Gratton, 1963), equivalent to $M_{v} \sim-12^{m}$ to $-15^{m}$. Burchell, around 1825 , seems to have been the first to find any irregularity in the star's light (Innes, 1903). Figure 4 shows the first published light curve

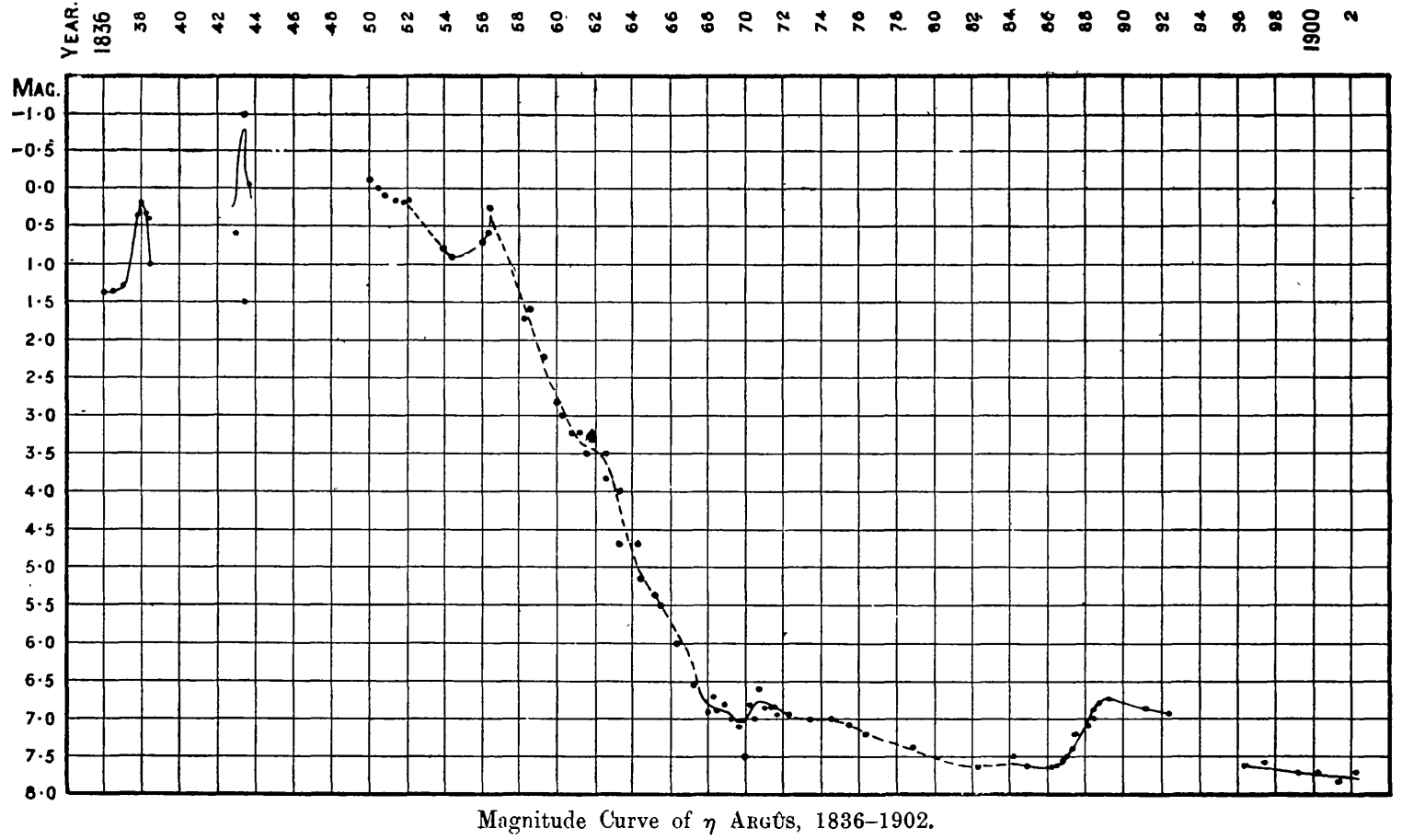

Fig. 4. The first published light curve of $\eta$ Car by Innes (1903).

constructed by Innes. Outbursts presumably also occurred in previous centuries even back to $\sim 1000 \mathrm{AD}$ in view of a number of isolated nebulosities at distances up to 25", which recede from $\eta$ Car (Walborn et al., 1978). At the time of Ptolemy in Alexandria (AD 135) the star was apparently not bright enough to be mentioned in the Almagest. Since the deep brightness minimum around 1880, the object has slowly increased in brightness and is increasing still. At present it is a 6th magnitude elliptical object, of which the reflection nebula is called the 'homunculus' (little human being; see the long exposures in Figure 5) by Gaviola (1950), with a visual diameter of $\sim 15^{\prime \prime}$ along the major axis. This homunculus is still expanding according to Gaviola (1950) and Ringuelet (1958) at a rate of some hundredths of a second per year (Figures 6 and 7). Figure 7 also shows the isophotes according to Gaviola.

The first spectra of $\eta$ Car were made around 1895, showing an F-type supergiant spectrum with strong P Cygni profiles, indicating high expansion velocities (Whitney, 1952). Good reproductions of these Harvard spectrograms (made at the Harvard Southern Station in Peru) are given by Walborn and Liller (1977). The F-type spectrum does not necessarily mean that the star itself is an F-type star. It could well be an Oor B-type star, surrounded by a dense cool expanding circumstellar gas envelope, with a spectrum which mimics that of an F-type supergiant. In the beginning of the 20th century the absorption lines were quickly replaced by emission lines (Cannon, 1916) 


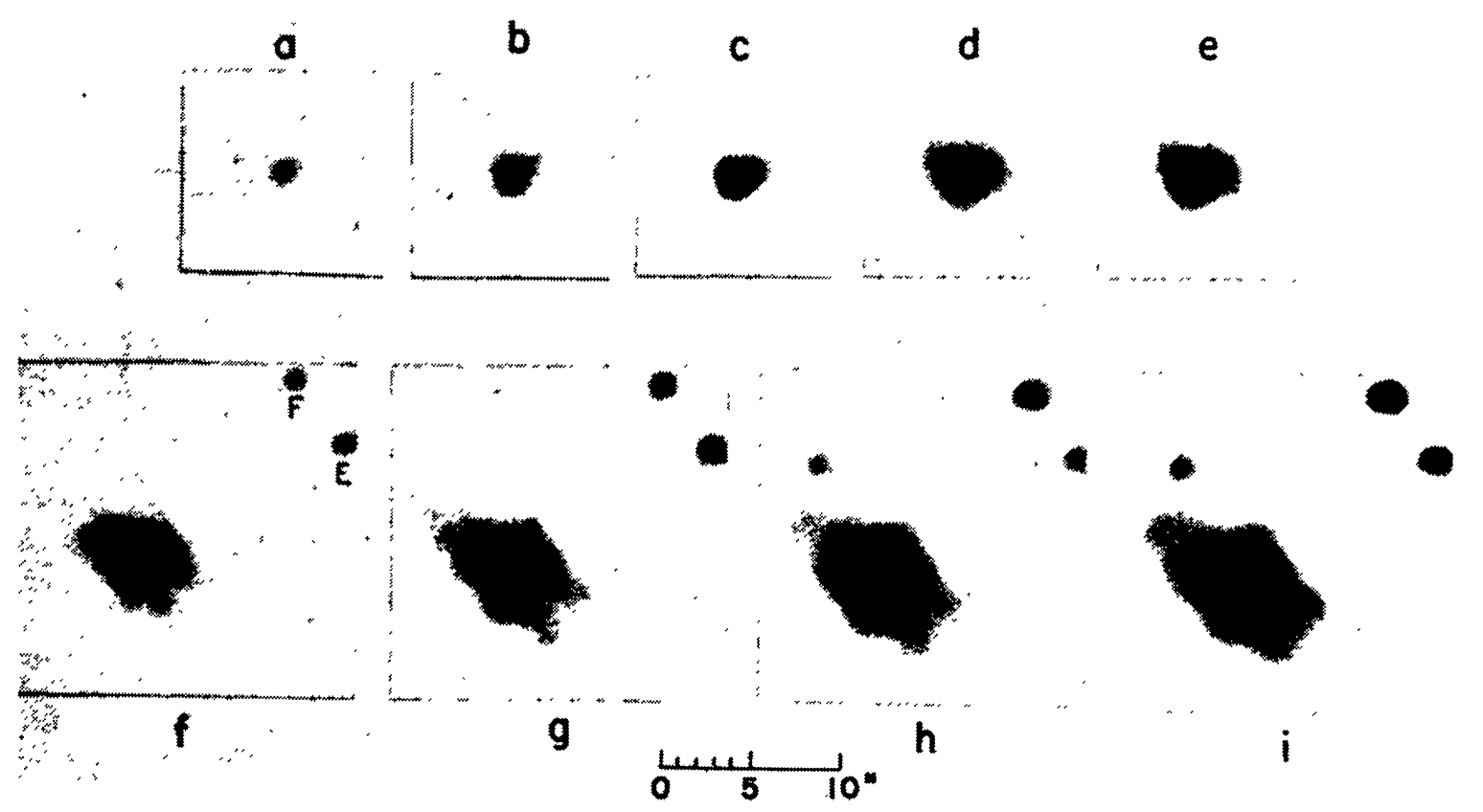

Fig. 5. A series of photographs of $\eta$ Car, doubling the time on successive exposures (from 1-256 s) made by Gaviola in 1944 with the 61-inch reflector at Bosque Alegre. $\mathrm{N}$ is at the top, $\mathrm{E}$ to the right. Courtesy Gaviola (1950).

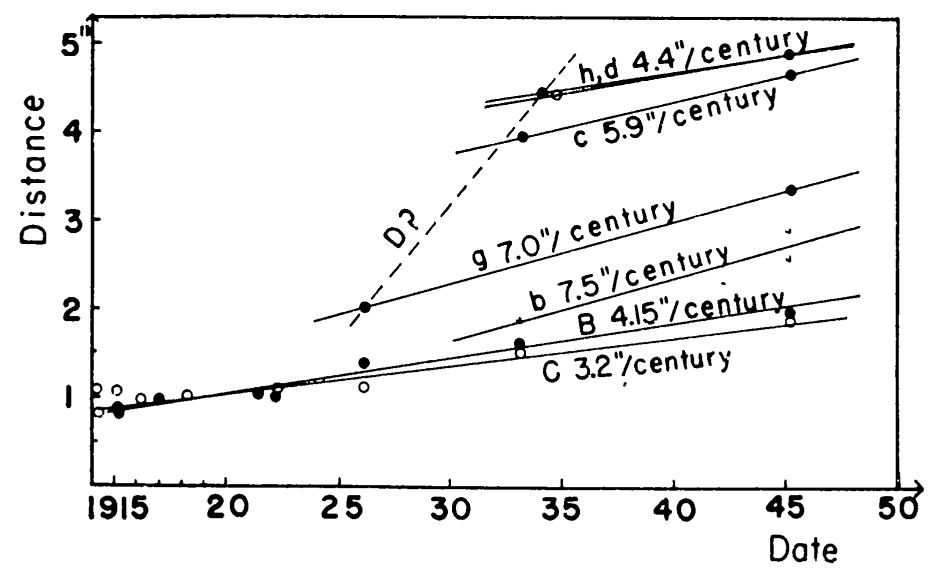

Fig. 6. The displacements of the condensations determined by Gaviola. Courtesy Gaviola (1950).

which is still the case (Aller, 1970). At present $\eta$ Car exhibits one of the most extraordinary display of emission lines among stellar spectra.

The temperature of the central star is probably very high, perhaps of the order of $30000 \mathrm{~K}$ according to the spectral considerations by Davidson (1971) based on the $\mathrm{H} \beta$ luminosity and by Andriesse et al. (1978) based on the presence of Fe III, N III, and $\mathrm{Ne}$ II lines. Also the X-ray emission detected by Chlebowski et al. (1984) points in that direction.

In the early sixties the distance of $\eta$ Car was estimated to be $\sim 1500 \mathrm{pc}$ (Gratton, 1963), but it is now taken to be twice as large (Sher, 1965; Feinstein et al., 1973; 


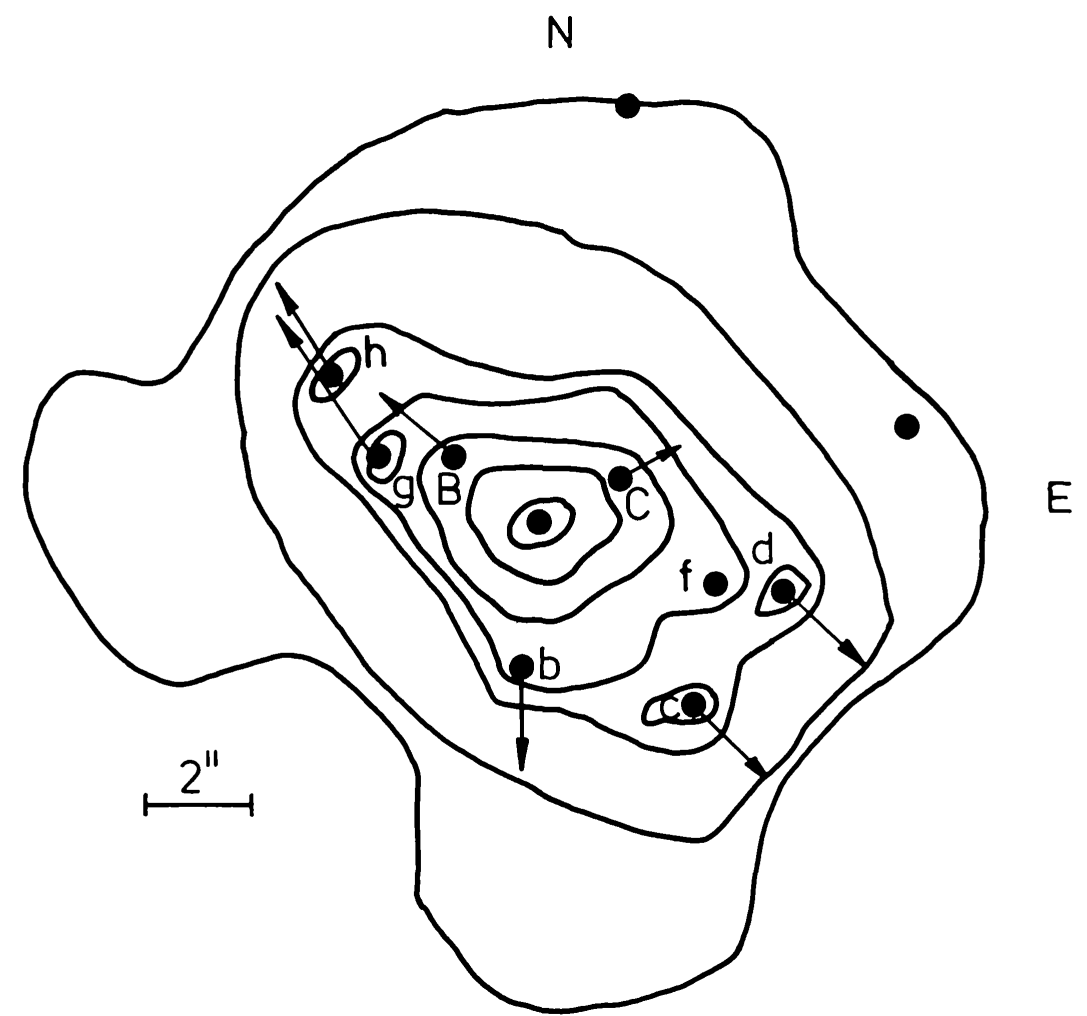

Fig. 7. The isophotes of $\eta$ Car according to Gaviola (1950) and the motions of the condensations according to Ringuelet (1958).

Walborn and Hesser, 1975; The et al., 1980a). As a result the total luminosity which follows from an integration of the spectral energy distribution corrected for foreground reddening appears to be:

$$
L \sim 6.6 \times 10^{6} L_{\odot}
$$

or

$$
M_{\mathrm{bol}} \sim-12^{m} \cdot 3,
$$

of which more than $90 \%$ is received from the infrared. This is known from the infrared observations, which show that $\eta$ Car is one of the most extreme infrared sources in the sky (e.g. Neugebauer and Westphal, 1968; Westphal and Neugebauer, 1969; Robinson et al., 1973; Aitken and Jones, 1975). Robinson et al. (1973) have shown that this very large infrared excess is due to thermal radiation of two dust shells surrounding the object. The dust particles are apparently heated by the ultraviolet photons of a very hot star in the very centre of the homunculus.

A large part of the decrease in brightness by $\sim 9^{m}$ after the 1843 maximum, was interpreted by Andriesse et al. (1978) in terms of a condensation rate into dust. The necessary mass loss rate should then be $0.08 M_{\odot} \mathrm{yr}^{-1}$, which is very high, and the total mass of the homunculus is estimated to amount to $\sim 10 M_{\odot}$. Hyland et al. (1979) arrived at a lower rate viz. $0.02 M_{\odot} \mathrm{yr}^{-1}$, which was based on a dust model of Mitchell 
and Robinson (1978). The total mass for the dust in the homunculus should then be $\sim 0.03 M_{\odot}$ and the total mass of the homunculus $\sim 3 M_{\odot}$.

Thackeray (1956) discovered the polarization of the homunculus by photographic means. The polarization of the homunculus as a whole has been measured photoelectrically by Visvanathan (1967) including the $\mathrm{H} \alpha$ emission line. Warren-Smith et al. (1979) obtained measurements of the optical linear polarization with better than 1 " resolution.

Interesting models of the close neighbourhood of $\eta$ Car based on this study and on the infrared radiation by Hyland et al. (1979) are available. They postulate the existence of a dust ring, which is illuminated by the central star seen nearly edge on surrounding the core (e.g. Figure 15). In the outer regions, the dust takes a roughly ellipsoidal shape, coinciding with the optical visible homunculus. Warren-Smith et al. postulate that the circumstellar ring has been formed by overflow from the outer Lagrangian point of a close binary star.

Some of the results of the following papers applying various observational techniques will also be mentioned or used in our discussion. Meaburn et al. (1983) havie shown from speckle observations of $\eta$ Car that it is an unresolved source: $<0.03$ arc sec $\equiv 84 \mathrm{AU}$. They suggest that it is a single star of $\sim 100 M_{\odot}$. Whitelock et al. (1983) have made $J H K L$ photometric observations of $\eta$ Car. Furthermore, they have obtained $C V F$ observations in the near infrared region between 1.2 to $4.2 \mu \mathrm{m}$. They have found that between 1972 and 1980 the star has been brightening slowly with a total range of $\sim 0^{m} 4$ in $\mathrm{K}$. Eta Car has been studied by Retallack (1983) using a high resolution aperture-synthesis radio telescope operating at $1415 \mathrm{MHz}$. In this wavelength region they found this object to be a discrete source having a flux density of $0.9 \pm 0.3 \mathrm{Jy}$. Ultraviolet spectral observations of $\eta$ Car employing the IUE by Cassatella et al. (1979) and high resolution observations of its $\mathrm{H} \alpha$ profile by Melnick et al. (1982) using the Coudé Echelle spectrograph fed by the ESO Coudé Auxiliary Telescope, show that $\eta$ Car has ejected three massive shells of matter in the past. Recent X-ray studies from $\eta$ Car were made by Chlebowski et al. (1984). The spectral data indicate two components: a soft one, which is characterized by a temperature of $5 \times 10^{6} \mathrm{~K}(0.4 \mathrm{keV})$ and a strongly absorbed hard one with a temperature of $8 \times 10^{7} \mathrm{~K}(7 \mathrm{keV})$, which they associate with the expanding outer shell and the central object, respectively. The derived parameters of the stellar wind from this object are typical for massive hot stars. It is likely that $\eta$ Car produces most of the energetic X-rays in the Carina Nebula, but this amounts to only $10^{-6}$ of its own total luminosity.

Concerning the evolutionary status, one may be rather sure that the star is in a post-Main Sequence stage in view of the overabundance of $\mathrm{N}$ and high He content of the ejected material (Davidson et al., 1982, 1984).

From 1974 to 1983 we have monitored $\eta$ Car photometrically in the VBLUW system. In addition to making this long series of measurements we also performed (in June 1983) a scanning of the homunculus avoiding the central core, and have made multiple aperture observations centred on the core. In the present paper we compare the variable behaviour of $\eta$ Car in the visual with that in the near infrared studied by Whitelock et al. 
(1983). Furthermore, an attempt is made to disentangle the photometric characteristics of the homunculus from that of the central core. An interpretation of the variability and a general discussion of the problems concerning $\eta$ Car and other S Dor type stars is then given.

\section{The Observations and Reductions}

The observations were made with the simultaneous Walraven VBLUW photometer attached to the $90 \mathrm{~cm}$ Light Collector at the Leiden Southern Station (the SAAO annex) in South Africa from 1974 to 1978, and after the telescope was moved to Chile at the ESO from 1979 to 1983 . A description of the photometer and the photometric system is given by Walraven and Walraven (1960), Rijf et al. (1969), and Lub and Pel (1977). The diaphragm used was $16^{\prime \prime}$; practically the whole homunculus fits in this diaphragm. Usually $\eta$ Car was measured four times alternately with the comparison star HDE 303311 (Sp. O5 V).

In the night of $7 / 8$ June 1983 (JD 2445493.542) the object was measured with four different diaphragms (denoted D1 to D4) centered on the core. Their apertures are listed in Table I, togehter with the ratio of the entrance surfaces with that of D1 (O1) as unit. On our request in the night of 24/25 June 1983 (JD 2445510.521) E. de Geus scanned the nebula along the declination axis with D1 avoiding the core. The sky was measured nearby the comparison star HDE 303311. Corrections for differential extinction were applied if necessary.

TABLE I

Diameters and surfaces of the four diaphragms

\begin{tabular}{lcc}
\hline Diaphragm & $\begin{array}{l}\text { Diameter } \\
(\operatorname{arc~sec})\end{array}$ & $\begin{array}{l}\text { Size of } \\
\text { entrance surfaces }\end{array}$ \\
\hline D1 & 5.8 & $\mathrm{O} 1$ \\
D2 & 8.3 & $2 \mathrm{O} 1$ \\
D3 & 11.6 & $4 \mathrm{O} 1$ \\
D4 & 16.5 & $8 \mathrm{O} 1$ \\
\hline
\end{tabular}

Due to improvements performed on the photometric system since the Light Collector was moved to Chile, there are slight systematic differences, which can be eliminated using transformation formulae determined by Pel (1980). It should be noted that all observations made after 1978 were transformed to the photometric system of $1970 / 1978$.

The comparison star HDE 303311 was calibrated with respect to standard stars on numerous occasions to derive its photometric data on the VBLUW system. Note that in this system, brightness and colours are expressed in terms of the logarithm of the intensity and intensity ratios, respectively. Table II gives the photometric data of HDE 303311 with the mean errors. 
TABLE II

Photometric parameters of the comparison star HDE 303311 (1970/1978 system)

\begin{tabular}{lrcrrrr}
\hline$V$ & $V-B$ & $\begin{array}{c}B-U \\
\text { (log intensity) }\end{array}$ & $U-W$ & $B-L$ & $V_{\mathbf{J}}$ & $(B-V)_{\mathbf{J}}$ \\
& & & & \multicolumn{2}{c}{$(\mathrm{mag})$} & \\
\hline-0.865 & 0.063 & -0.017 & 0.021 & 0.005 & 9.031 & 0.165 \\
\pm 0.002 & \pm 0.002 & \pm 0.002 & \pm 0.003 & \pm 0.002 & \pm 0.005 & \pm 0.005 \\
\hline
\end{tabular}

The $V-B$ colour index of the $V B L U W$ system can be transformed into the equivalent $B-V$ colour index of the Johnson $U B V$ system (denoted by the subscript $\mathrm{J}$ ) using Table VII in Walraven et al. (1964) and revised according to a formula given by Lub and Pel (1977). The visual magnitude of the $U B V$ system, $V_{\mathrm{J}}$, can be obtained from the $V$ and the $V-B$ of the $V B L U W$ system using the transformation formula given by Pel (1976). Table III lists the photometric data of $\eta$ Car made with the 16" diaphragm (D4) from 1974 to 1983 . Table IV gives the multiple aperture observations centered on the core. Note that the observation in this series made with D4 is not listed in Table III. Table $\mathrm{V}$ lists the results of the scan made with $\mathrm{D} 1$, the positions are numbered 1 to 10 . They are sketched in Figure 10. It should be mentioned that all data in Tables II to V are in the system 1970/1978.

The transformation formulae of Pel (1976) to obtain $V_{\mathrm{J}}$ and $(B-V)_{\mathrm{J}}$ are strictly speaking only applicable to a normal stellar spectrum. Since many emission lines are superimposed on the continuum of $\eta$ Car, differences with $U B V$ observations may amount to $0^{m} .1$.

Because of bad seeing conditions in 1974 to 1976, the scatter in the D4 measurements is rather large (see Figure 8), but otherwise they amount to \pm 0.008 for $V, \pm 0.005$ for $V-B, B-L$, and $B-U$ and \pm 0.010 for $U-W$. The errors in the D1 to D3 measurements (Table IV) are difficult to estimate, since they were only made once. They will be mainly dependent on the influence of seeing conditions, and on the pointing error of the telescope, which may amount to $1^{\prime \prime}$.

\section{The Light and Colour Curves}

Figure 8 shows the light and colour curves based on observations with diaphragm D4 from 1974 to 1983 . The data of the ultraviolet ANS observations published by Pottasch et al. (1976) is indicated by a thick arrow. At the right-hand side the $V_{\mathrm{J}}$ and $(B-V)_{\mathrm{J}}$ scales are added. Apart from the years 1974 to 1976, when bad seeing conditions strongly influenced the measurements, the light- and colour variations are smooth and show a number of maxima and minima with time scales of 2 to $3 \mathrm{yr}$. The general tendency in the $10 \mathrm{yr}$ time interval is a slow increase in visual and blue light by $\sim 0^{m} .4$ mag., while the rise in the ultraviolet bands $L\left(\lambda_{\text {eff }} \simeq 3840 \AA\right), U\left(\lambda_{\text {eff }} \simeq 3630 \AA\right)$, and $W\left(\lambda_{\text {eff }} \simeq 3250 \AA\right)$ are approximately $0.5,0.45$, and 0.4 mag. respectively. This is in very good agreement with what is found by Whitelock et al. (1983) in the near infrared. 
TABLE III

The photometric data of $\eta$ Car made with a $16^{\prime \prime}$ diaphragm (D4) in the VBLUW system (in $\log$ intensity scale) and in the $U B V$ system (in magnitude; $V$ and $B-V$ with subscript $J$ )

\begin{tabular}{|c|c|c|c|c|c|c|c|}
\hline $\begin{array}{l}\text { J.D.- } \\
2440000\end{array}$ & $V$ & $V-B$ & $\begin{array}{c}B-U \\
\text { (log intensity) }\end{array}$ & $U-W$ & $B-L$ & $V_{\mathbf{J}}$ & $(B-V)_{J}$ \\
\hline 2125.441 & 0.300 & 0.218 & 0.162 & 0.012 & 0.184 & 6.09 & 0.54 \\
\hline 2126.345 & 0.248 & 0.225 & 0.179 & 0.014 & 0.189 & 6.22 & 0.56 \\
\hline 2129.271 & 0.281 & 0.225 & 0.191 & 0.008 & 0.202 & 6.14 & 0.56 \\
\hline 2135.394 & 0.254 & 0.237 & 0.195 & 0.001 & 0.196 & 6.20 & 0.59 \\
\hline 2142.238 & 0.268 & 0.195 & 0.188 & -.018 & 0.212 & 6.18 & 0.49 \\
\hline 2144.256 & 0.284 & 0.222 & 0.173 & 0.007 & 0.186 & 6.13 & 0.55 \\
\hline 2145.439 & 0.283 & 0.231 & 0.179 & 0.016 & 0.193 & 6.13 & 0.57 \\
\hline 2146.319 & 0.233 & 0.216 & 0.189 & -.007 & 0.200 & 6.26 & 0.53 \\
\hline 2509.290 & 0.319 & 0.208 & 0.175 & -.005 & 0.193 & 6.05 & 0.51 \\
\hline 2510.326 & 0.288 & 0.208 & 0.190 & -.008 & 0.177 & 6.12 & 0.51 \\
\hline 2515.310 & 0.290 & 0.212 & 0.188 & -.002 & 0.152 & 6.12 & 0.52 \\
\hline 2522.416 & 0.266 & 0.225 & 0.202 & 0.018 & 0.217 & 6.18 & 0.56 \\
\hline 2523.329 & 0.278 & 0.214 & 0.196 & 0.010 & 0.213 & 6.15 & 0.53 \\
\hline 2528.363 & 0.240 & 0.220 & 0.210 & 0.001 & 0.221 & 6.24 & 0.54 \\
\hline 2533.305 & 0.261 & 0.220 & 0.198 & -.017 & 0.208 & 6.19 & 0.54 \\
\hline 2535.375 & 0.268 & 0.228 & 0.216 & 0.013 & 0.223 & 6.17 & 0.56 \\
\hline 2538.367 & 0.245 & 0.237 & 0.226 & 0.019 & 0.228 & 6.13 & 0.58 \\
\hline 2874.371 & 0.346 & 0.220 & 0.169 & 0.016 & 0.173 & 5.98 & 0.54 \\
\hline 2875.435 & 0.340 & 0.226 & 0.161 & 0.041 & 0.163 & 5.99 & 0.56 \\
\hline 2876.349 & 0.356 & 0.228 & 0.183 & 0.051 & 0.193 & 5.95 & 0.56 \\
\hline 2877.353 & 0.390 & 0.210 & 0.163 & 0.014 & 0.176 & 5.87 & 0.52 \\
\hline 2879.379 & 0.339 & 0.225 & 0.171 & 0.019 & 0.192 & 5.99 & 0.50 \\
\hline 2886.240 & 0.353 & 0.224 & 0.179 & 0.018 & 0.174 & 5.96 & 0.55 \\
\hline 2887.381 & 0.357 & 0.223 & 0.192 & 0.006 & 0.186 & 5.95 & 0.55 \\
\hline 2888.367 & 0.336 & 0.229 & 0.184 & 0.023 & 0.184 & 6.00 & 0.56 \\
\hline 2891.406 & 0.319 & 0.225 & 0.181 & 0.033 & 0.185 & 6.04 & 0.55 \\
\hline 2892.275 & 0.353 & 0.216 & 0.172 & 0.005 & 0.175 & 5.96 & 0.53 \\
\hline 2893.378 & 0.353 & 0.221 & 0.170 & 0.025 & 0.174 & 5.96 & 0.55 \\
\hline 2894.274 & 0.365 & 0.209 & 0.183 & 0.101 & 0.194 & 5.93 & 0.52 \\
\hline 2896.230 & 0.371 & 0.222 & 0.180 & 0.032 & 0.180 & 5.91 & 0.55 \\
\hline 2904.341 & 0.358 & 0.230 & 0.181 & 0.016 & 0.180 & 5.95 & 0.57 \\
\hline 3179.483 & 0.302 & 0.228 & 0.191 & 0.034 & 0.195 & 6.09 & 0.56 \\
\hline 3186.441 & 0.316 & $\approx 0.229$ & 0.185 & 0.028 & 0.190 & 6.05 & 0.56 \\
\hline 3187.441 & 0.300 & 0.233 & 0.194 & 0.041 & 0.198 & 6.09 & 0.58 \\
\hline 3189.427 & 0.308 & 0.228 & 0.192 & 0.022 & 0.193 & 6.07 & 0.56 \\
\hline 3190.476 & 0.315 & 0.223 & 0.177 & 0.013 & 0.181 & 6.05 & 0.55 \\
\hline 3198.431 & 0.321 & 0.222 & 0.182 & 0.022 & 0.183 & 6.04 & 0.55 \\
\hline 3203.441 & 0.313 & 0.229 & 0.187 & 0.025 & 0.189 & 6.06 & 0.56 \\
\hline 3220.365 & 0.311 & 0.235 & 0.186 & 0.014 & 0.186 & 6.06 & 0.58 \\
\hline 3231.424 & 0.313 & 0.234 & 0.186 & 0.021 & 0.188 & 6.06 & 0.58 \\
\hline 3248.337 & 0.320 & 0.231 & 0.174 & 0.020 & 0.183 & 6.04 & 0.57 \\
\hline 3260.382 & 0.306 & 0.238 & 0.188 & 0.032 & 0.193 & 6.07 & 0.59 \\
\hline 3270.247 & 0.315 & 0.229 & 0.173 & 0.013 & 0.183 & 6.05 & 0.56 \\
\hline 3284.323 & 0.308 & 0.226 & 0.176 & 0.017 & 0.185 & 6.07 & 0.56 \\
\hline 3298.289 & 0.296 & 0.219 & 0.184 & 0.021 & 0.198 & 6.10 & 0.54 \\
\hline 3301.228 & 0.296 & 0.221 & 0.183 & 0.005 & 0.193 & 6.10 & 0.55 \\
\hline 3303.267 & 0.299 & 0.223 & 0.187 & 0.012 & 0.198 & 6.09 & 0.55 \\
\hline 3307.231 & 0.298 & 0.222 & 0.182 & 0.012 & 0.191 & 6.10 & 0.55 \\
\hline
\end{tabular}


Table III (continued)

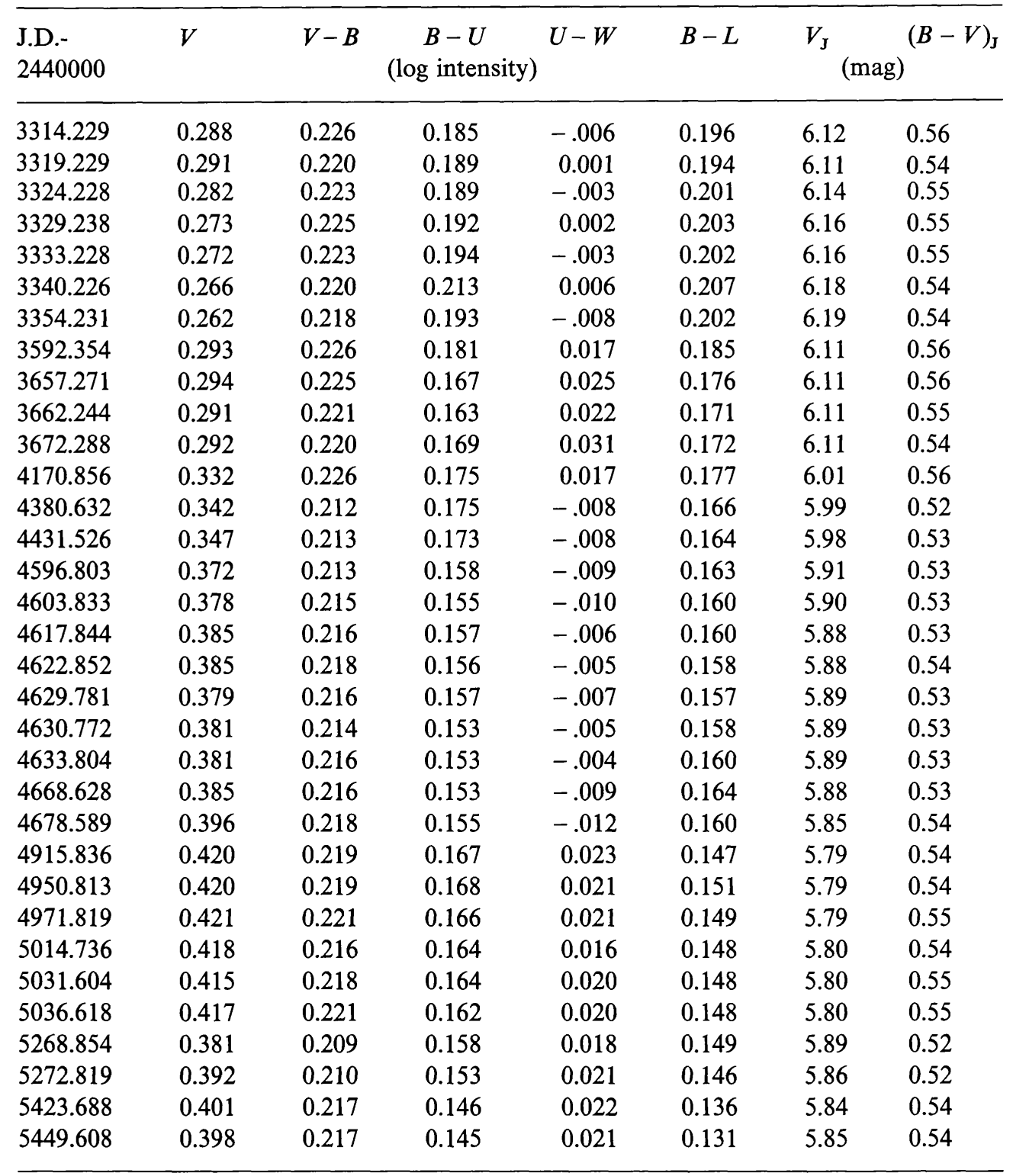

TABLE IV

Photometric data for $\eta$ Car made with four different diaphragms, centered on the central object (on JD $2445493.542=7 / 8$ June, 1983)

\begin{tabular}{llllllll}
\hline Diaphragm & $V$ & $V-B$ & $\begin{array}{c}B-U \\
\text { (log intensity) }\end{array}$ & $U-W$ & $B-L$ & $V_{\mathbf{J}}$ & $(B-V)_{\mathbf{J}}$ \\
$(\mathrm{mag})$ & \\
\hline D1 & 0.045 & 0.279 & 0.275 & 0.146 & 0.249 & 6.72 & 0.68 \\
D2 & 0.289 & 0.228 & 0.173 & 0.042 & 0.170 & 6.12 & 0.56 \\
D3 & 0.357 & 0.222 & 0.150 & 0.023 & 0.148 & 5.95 & 0.55 \\
D4 & 0.400 & 0.222 & 0.141 & 0.021 & 0.136 & 5.84 & 0.55 \\
\hline
\end{tabular}

It is of interest to note that the colour indices $B-L, B-U$ and $B-W$ run parallel to the visual light curve, whereas $V-B$ generally runs in antiphase and that the latter does not show a net change in a decade (see Section 9.6). 


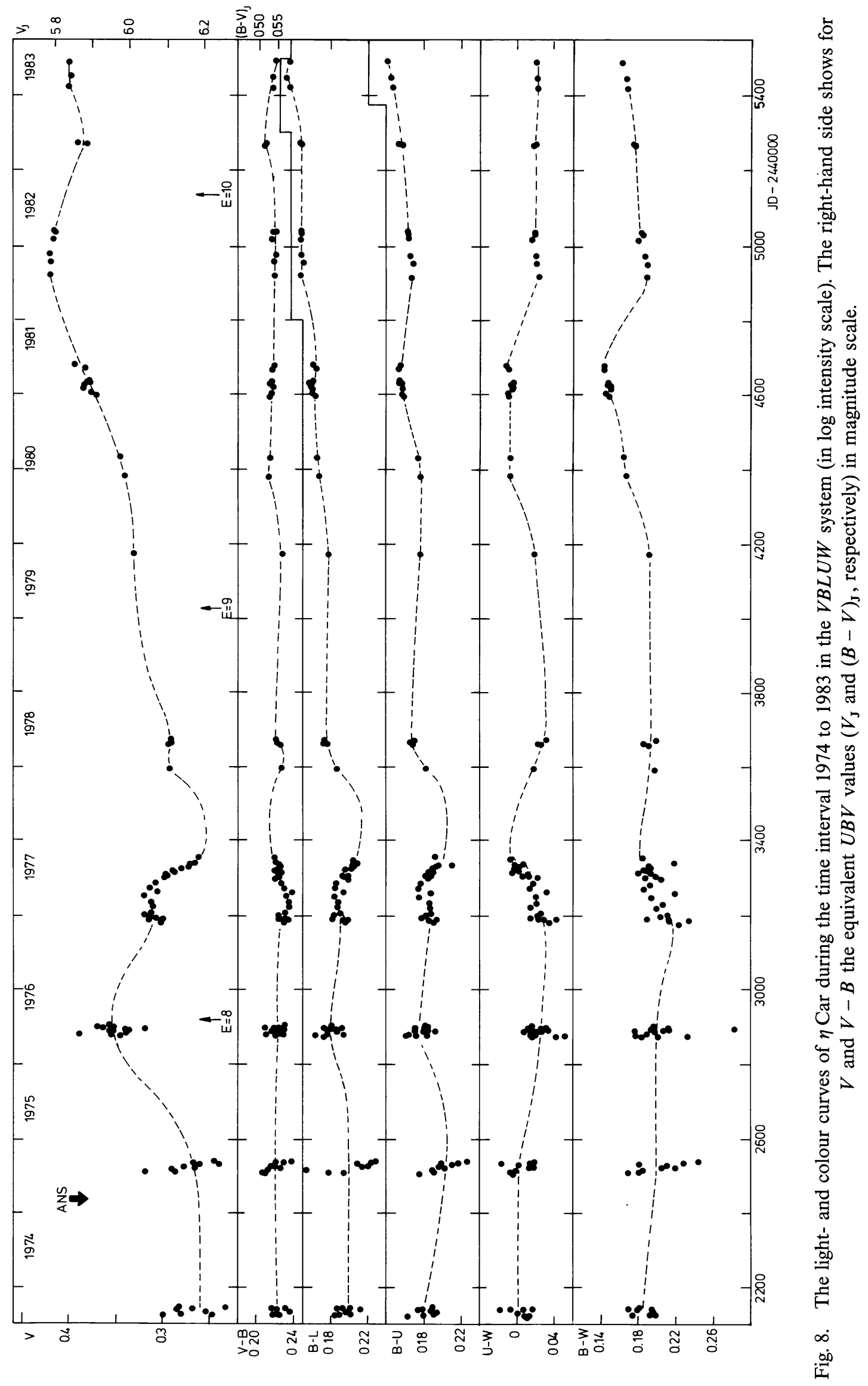


Feinstein and Marraco (1974) suggested the possible presence of a three year cycle in the light curve. Using their formula for the ephemeris of the maximum:

$$
\mathrm{JD}_{\max }=\mathrm{JD} 2434037+1110^{\mathrm{d}} E
$$

we obtained with $E=8,9$, and 10 the dates indicated by the three thin arrows in the upper part of Figure 8. The position of the first arrow fits very well with the 1976 maximum. The second falls in a part of the light curve which lacks sufficient observations. However, the infrared light of Whitelock et al. (1983) shows two small maxima at both sides (see Figure 9). The third computed maximum comes a year too early. Thus we cannot confirm a strict periodicity of the occurrence of a maximum. At most one can speak of a characteristic period of 2 to $3 \mathrm{yr}$. Thus a binary hypothesis for the cause of this variation fails (see also Meaburn et al., 1983).

As a matter of interest we show in Figure 9 the schematic curves in $V_{\mathbf{J}}$ (transformed from $V$ ), and the infrared observations in the $J H K L$ system of Whitelock et al. In order to compare the relative amplitudes with each other, we have normalized all light curves at the $V_{\mathbf{J}}$ magnitude on JD 2443225. There is in general a good correlation between all light curve features. In fact our $V_{\mathbf{J}}$ curve matches the infrared curves much better than the one based on the visual estimates by Bateson (1972-1982), such as is published by Whitelock et al. (1983), illustrating the difficulty of estimating the brightness of an extended object visually. Small relative differences illustrate the varying physical conditions of the object. The major difference is exhibited by the light curve in $\mathbf{J}(1.25 \mu \mathrm{m})$, which dropped suddenly by $\sim 0^{m} .2$ below the other curves around JD 2443400 and stayed lower by this amount until now. However, it is also possible to say that at the $J$ passband the object is brighter by about 0.2 before and around JD 2443200, and behaves up till now like at the other passbands.

It is important to question the influence of emission lines situated in the wavelength intervals of the various passbands. The $J$ passband contains the prominent Paschen $\beta$ line in emission (Whitelock et al., 1983). The $H$ passband contains the Brackett 10, 11, 12 , and 13 emission lines, and the $K$ passband that of Bracket $\gamma$ and He I. According to Whitelock et al. the contribution of the emission lines to the total flux in the $J$ band is only $3 \%$ and in the $K$ band $2 \%$. In the $H$ band the contribution of the Brackett emission lines will be of the same order of magnitude. Therefore, the major source of the light variation of $\eta$ Car is its continuum. This continuum underwent in 1981 the largest variation of the last $10 \mathrm{yr}$. It was largest in $J, H$, and $K$ and smallest in $L$, whereas in $V_{\mathrm{J}}$ it was intermediate.

\section{The Two-Colour Diagrams and the Photometric Parameters of the Homunculus and the Central Object}

\subsection{THE TWO-COLOUR DIAGRAMS}

Figure 10 shows the three intrinsic two-colour diagrams of the $V B L U W$ system partly. The observed positions of $\eta$ Car as measured with diaphragm D4 is schematically indicated for 3 intervals of years: 1974/1976, 1977/1978, and 1979/1983, indicated by 


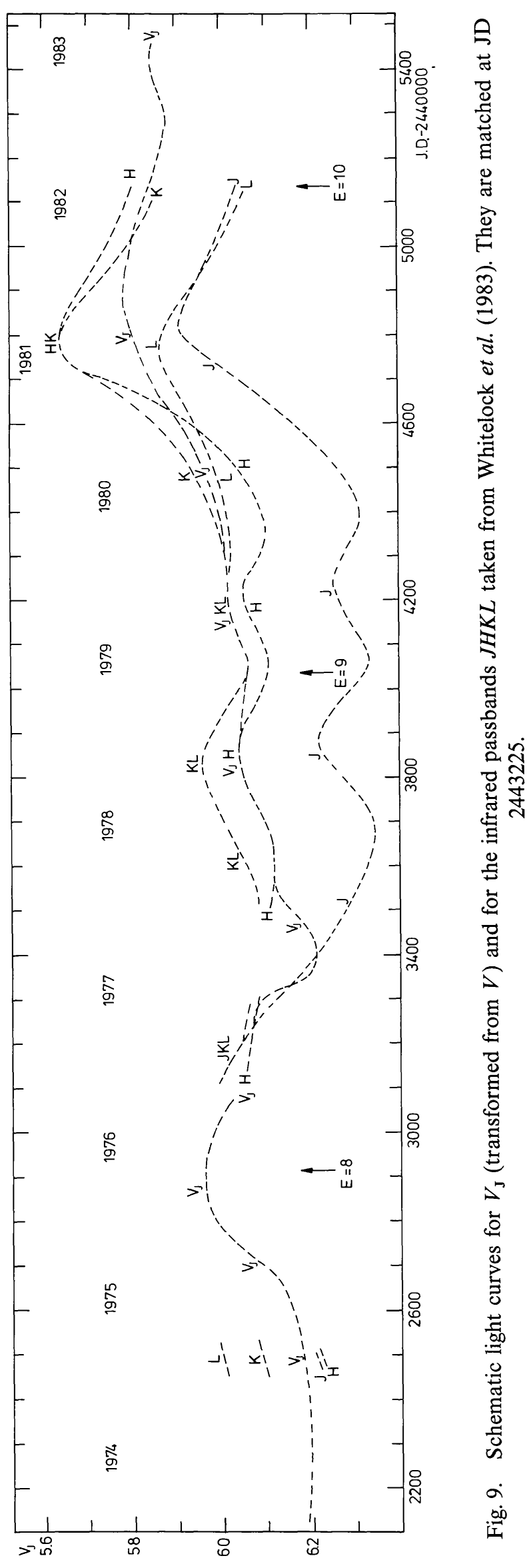




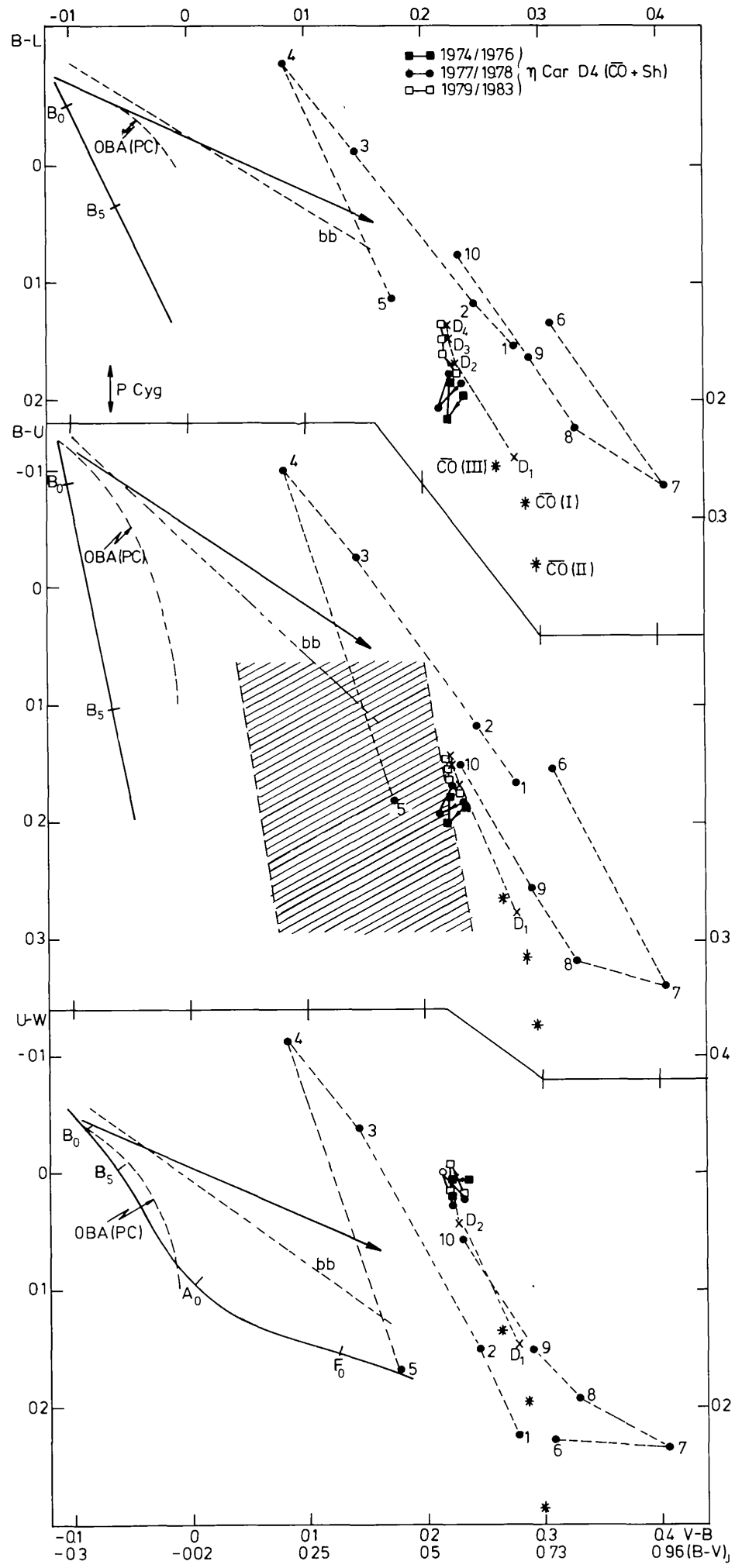

Fig. 10. The three two-colour diagrams with the position of $\eta$ Car and the nebulosity (dots, numbered 1 to 10). The long straight arrow is the reddening line. As a matter of interest the supposed position of $\mathrm{P}$ Cyg on the OBA(PC) relation is indicated. For a further description of this figure see Section 4. 
black squares, black dots and open squares, respectively. In these diagram are also shown: the reddening directions (long arrows), the black-body lines (dotted) and the intrinsic relations for OBA type super- and hypergiants with $\mathbf{P}$ Cygni profiles in the optical spectrum (dotted curve indicated by OBA(PC); van Genderen et al., 1983). These latter relations will be used for comparison purposes. The $V-B$ vs $B-U$ diagram also indicates the observed positions (hatched area) of the members of the open cluster Tr 16 (to which $\eta$ Car also belongs) based on $V B L U W$ photometry by Thé et al. (1980a).

Since it is important for the rest of the discussion to differentiate between the various components of the object $\eta$ Car, we explain at the right top explicitely that the measurements through D4 includes both the contribution of the central object obscured by the intervening material of the surrounding homunculus, $(\overline{\mathrm{co}})$, and the light of the homunculus or shell (sh).

Let us now examine in detail the behaviour of the colour indices of $\eta$ Car. The slow change of the colours in $B-L$ and $B-U$ is clearly visible: the object becomes more ultraviolet in these indices. Furthermore, in view of its position above the black-body relation in the $U-W$ vs $V-B$ diagram, $\eta$ Car has at the $W$ pass-band $\left(\lambda_{\text {eff }} \sim 3250 \AA\right)$ clearly an excess of radiation compared to the $U$ band $\left(\lambda_{\text {eff }} \sim 3650 \AA\right)$ by $\sim 0$. 3 . The absence of any net change in $V-B$ and $U-W$ during the interval 1974-1983 can be seen in Figure 8. The position of $\eta$ Car just on the right-hand border of the above mentioned $\operatorname{Tr} 16$ hatched area indicates that the reddening suffered by the object is of the same order (Section 5). However, before drawing a definite conclusion it is important to study the presence of the many emission features at all pass-bands, thus also in $V$ and $B$, which may have substantial influence to the colour index $V-B$ (Rodgers and Searle, 1967; Viotti, 1969; Aller, 1970). According to Rodgers and Searle these emission features contributes about $30 \%$ in the visual, whereas according to Whitelock et al. (1983) about $40 \%$ in the blue (see their Section 2) is caused by emission features. This means that in the $B$ there is a net emission of $\sim 10 \%$, in other words the Johnson $(B-V)_{\mathrm{J}}$ is too blue by $\sim 0^{m} .1$. (Viotti (1969) suggested $\sim 0^{m} .3$ ). We have estimated for the $V B L U W$ system using Rodgers and Searle's Table III and our Figure 8, that the colour index $V-B$ is too blue by roughly the same amount as in $(B-V)_{\mathrm{J}}$.

The photometric data of $\eta$ Car made with the smaller diaphragms in June 1983 and centered on the core (Table IV, Figure 11) are indicated with crosses and connected with thin lines. The result is interesting: the central area dominated by $(\overline{\mathrm{co}})$ (see below and Section 4.2) appears to be redder in all colour-indices than the homunculus. From D4 to $\mathrm{D} 1,(B-V)_{\mathrm{J}}$ becomes redder by $0^{m} .13$. Although the entrance surface decreases by a factor of 8 (see Table I), the visual magnitude $V_{\mathrm{J}}$ decreases by $1^{m}$ only (see Table IV). This indicates that the light received in D1 is dominated by $(\overline{\mathrm{co}})$ (see also Section 4.2 ).

The results of the scanning are shown also in Figure 10. They are connected by dotted lines. Those made to the east of the core are numbered 1-5, while those to the west 6-10 (see Table V). The precise positions of D1 are sketched in Figure 11 on scale. The isophotes of the homunculus and the condensations (dots) are taken from Gratton (1963) and Ringuelet (1958). The results of the scanning reveal two major points of 


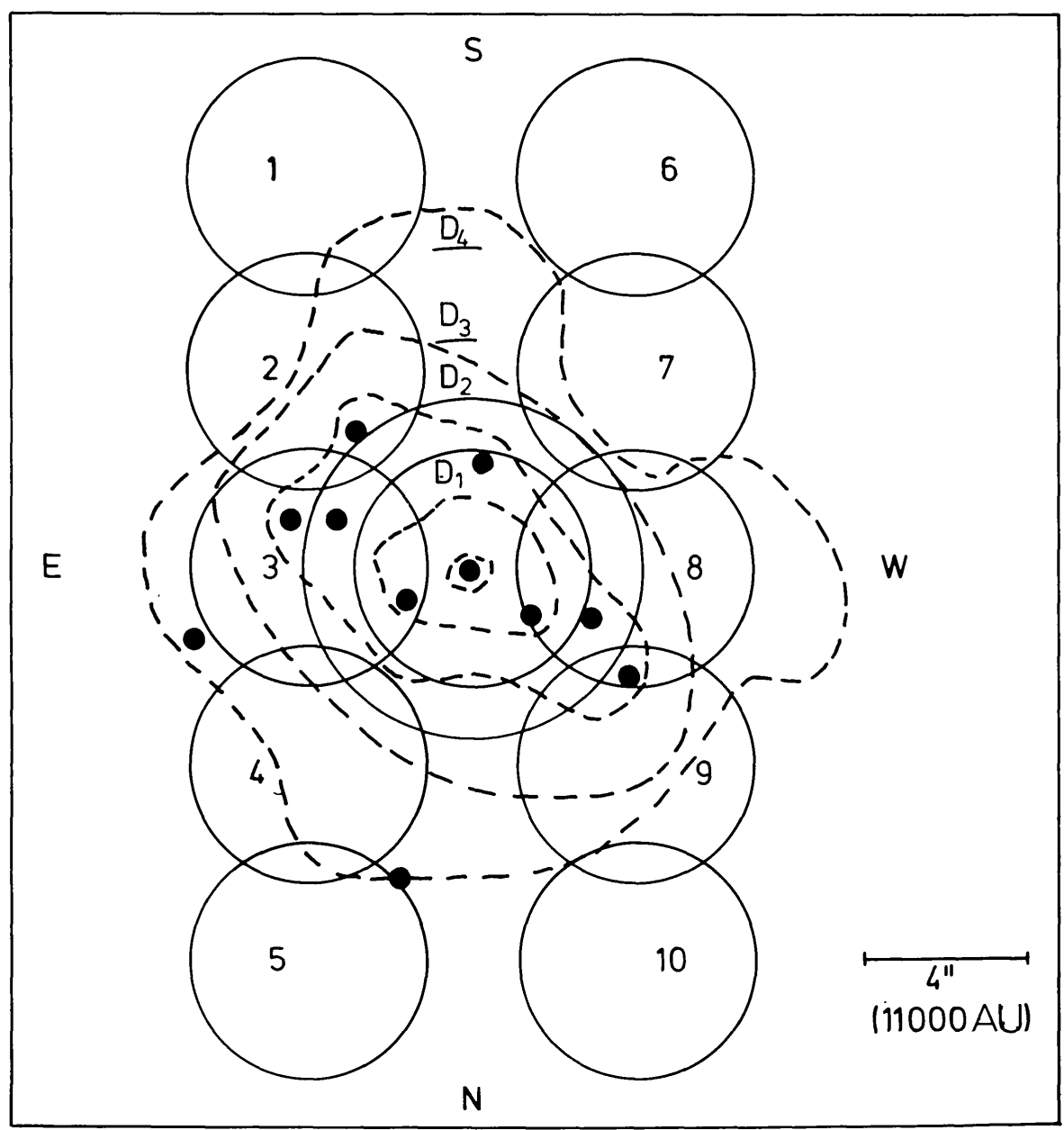

Fig. 11. The schematical positions of the diaphragms used for the central area (D1 to D4) and used for the scanning of the nebulosity (positions numbered 1 to 10), superimposed on the outline of a number of isophotes of $\eta$ Car. In order not to confuse the figure, the edges of the diaphragms D3 and D4 are only sketched with a short bow. The dots are condensations according to Gratton (1963).

interest. (1) There is a remarkable large spread in colour in the homunculus. Note, however, that the slopes of the lines connecting subsequent positions are roughly constant. (2) The positions to the east of the north-south line are generally bluer and even surpass the black body line (positions 3 and 4), while those to the west are significantly redder by $\sim 0^{m} .4$ in $(B-V)_{\mathrm{J}}$ as well as in other colour indices. Uncertainties may amount to $\sim 0^{m} .2$ in the colour indices or $\sim 0.1$ in log intensity scale.

As can be seen in Table $V$, the positions 1, 5, 6, and 10, situated just outside the bright part of the homunculus, are the faintest, their $V_{\mathrm{J}}$ ranges $12^{m}$ to $13^{m} .5$. Since the integrated visual brightness of $\eta$ Car is $\sim 6^{m}$, a large amount of straylight must be present in the measurements of these exterior positions. It is therefore not surprising that their places in the two colour diagrams lie close to that of the D4 measurements. Because of this reason we shall omit them in our further discussion. The most important results are those of the positions 2,3 , and 4 at the east and 7, 8, and 9 at the west side of the north-south line. Their integrated visual brightnesses are large enough $\left(9^{m}-10^{m} \cdot 7\right)$ so that 
TABLE V

Results of the scanning of the nebulosity with D1 with shifts in declination of 4 " (made on JD $2445510.521=24 / 25$ June, 1983)

\begin{tabular}{|c|c|c|c|c|c|c|c|}
\hline Position & $V$ & $V-B$ & $\begin{array}{r}B-U \\
\quad(\log \end{array}$ & $\begin{array}{l}U-W \\
\text { ensity) }\end{array}$ & $B-L$ & $V_{\mathrm{J}}$ & $(B-V)_{\mathrm{J}}$ \\
\hline 1 & -2.376 & 0.278 & 0.165 & 0.224 & 0.154 & 12.77 & 0.68 \\
\hline 2 & -1.083 & 0.245 & 0.116 & 0.150 & 0.117 & 9.54 & 0.61 \\
\hline 3 & -0.882 & 0.143 & -0.026 & -0.039 & -0.013 & 9.06 & 0.36 \\
\hline 4 & -1.517 & 0.082 & -0.101 & -0.113 & -0.087 & 10.66 & 0.20 \\
\hline 5 & -1.891 & 0.175 & 0.181 & 0.168 & 0.113 & 11.58 & 0.44 \\
\hline 6 & -2.705 & 0.309 & 0.153 & 0.227 & 0.134 & 13.58 & 0.75 \\
\hline 7 & -1.441 & 0.406 & 0.341 & 0.235 & 0.273 & 10.41 & 0.96 \\
\hline 8 & -0.901 & 0.330 & 0.317 & 0.193 . & 0.223 & 9.08 & 0.79 \\
\hline 9 & -0.975 & 0.291 & 0.256 & 0.150 & 0.163 & 9.27 & 0.71 \\
\hline 10 & -2.468 & 0.231 & 0.150 & 0.056 & 0.076 & 13.01 & 0.57 \\
\hline
\end{tabular}

it is sure that the colour differences are real. To be completely certain, we searched for possible colour effects in the measurements made with the smallest diaphragm. Therefore J. Brand measured on our request in April 1984, a few times two stars with different diaphragms. It appeared that colour effects do exist for D1, but they amount to at most 0.009 in $\log$ intensity scale (for $B-L$; the effect in the other colour indices is even smaller), so that they can be ignored.

The positions 2 and 9, 3 and 8 , and 4 and 7 are situated symmetrically with respect to the core. It is interesting to see that the $V_{\mathrm{J}}$ magnitudes per pair are nearly equal: $9^{m} \cdot 4$, $9^{m} .1$, and $10^{m} .5$, respectively, with deviations not much more than $\pm 0 .{ }^{m} 1$ within each pair. This means that the setting on the visible nebulosity has been done satisfactory. The ultraviolet excess coming from the positions 3 and 4 (if real), might be caused by emission radiation of ionized gas. In this respect it is important to note that the location of $\mathrm{H}$ II regions in the two-colour diagrams of the VBLUW system is also above the black-body relation (Greve et al., 1982). The asymmetry in the colours (at the east bluer than at the west of the core) cannot yet be explained. It would be of importance to confirm this asymmetry by means of a difference between the isophotes for different passbands. The isophotes from red to ultraviolet measured by Walborn and Gull (1982) extend slightly further to the east- than to the west-side of the core. However, one would like to know how the isocolour lines run. The asymmetry of the colours suggest that the homunculus is not built up homogeneously. This is obvious from Figure 12, a computerized image of $\eta$ Car $(\lambda=6000 \AA)$ made by Davidson. Bi-polar reflection nebulae are highly inhomogeneous and apparently this may also refer to the homunculus, because of a possible similar outflow pattern (Icke, 1981).

\subsection{THE PHOTOMETRIC PARAMETERS OF THE CENTRAL OBJECT AND THE HOMUNCULUS}

Since measurements of the central area and of the homunculus are made with the same diaphragm (D1), it is in principle possible to estimate true photometric parameters of 


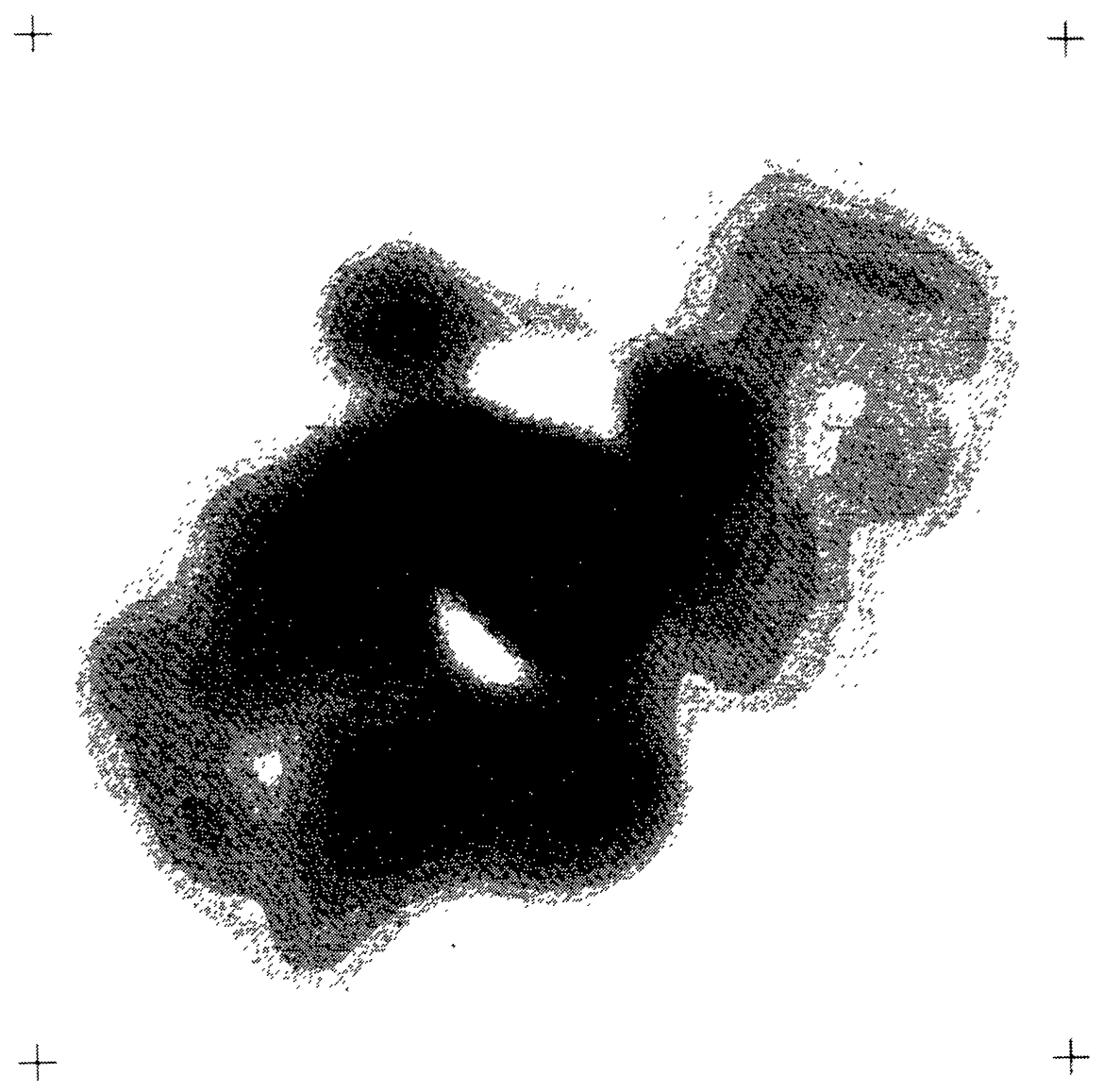

Fig. 12. A computerized continuum image of $\eta$ Car made by $\mathrm{K}$. Davidson at the Cerro Tololo InterAmerican Observatory in February 1981 and kindly put by him at our disposal. Use has been made of a $1.5 \mathrm{~m}$ telescope equipped with a SIT vidicon system. An interference filter with a width of $100 \AA$ was used and centered at $6000 \AA$. Significant emission features are therefore excluded. The total dynamical range in the picture is large, approximately $4000 \times$ larger in the peak of the center than at the outer contour. The intensity range is standardized by measuring nearby stars. The contour interval is of the order of 0 . 5 . The crescent like spots of 'low-intensity' to the north and the south-east of the central peak are probably an artifact of the resolution-enhancement program and therefore presumably not real. $\mathrm{N}$ is at the top and $\mathrm{E}$ to the left.

the central object $(\overline{\mathrm{co}})$ by subtracting a suitable homunculus measurement (sh) from that of the central area. For the homunculus measurement several modes can be chosen. (1) One can use the average measurement at the positions 3 and 8 (see Figure 11), which lie closest to the D1 measurement. Note that they have some overlap with D1, covering $\sim 2 / 5$ of its surface. It is important to remark here, that although the colour difference between positions 3 and 8 is rather large, the effect on the result for the core will not be of much importance, because ( $\overline{\mathrm{co}}$ ) is $\sim 2^{m}$ brighter than the homunculus measured through D1 (see Tables V and VI). In fact, the visual brightness of the central area 
measured through D1 is $2^{m} \cdot 4$ brighter than the average of those of the positions 3 and 8 (see Tables IV and V). (2) Since it is possible that the homunculus increases in brightness towards the centre, the average brightness of positions 3 and 8 , if used for correction, is possibly an under-estimate. How large this increase is, is not known. Therefore, we will assume as a second mode, that the brightness of the homunculus entering D1 is equal to twice that of the average of the positions 3 and 8. (3) In order to get more insight in the effect of the place of $(\overline{\mathrm{co}})$ in Figure 10, we will assume, as a third mode, that the measurement at position 8 is representative for the homunculus flux received in $\mathrm{D} 1$.

The results of the subtractions done for the three modes will be called $\overline{\mathrm{co}}$ (I), $\overline{\mathrm{co}}$ (II), and $\overline{c o}(\mathrm{III})$, respectively. They are listed in Table VI, and are plotted in Figure 10 as asterisks. The difference in the three positions reflects the uncertainty in the deduced parameters for $(\overline{\mathrm{co}})$. Note that $(\overline{\mathrm{co}})$ represents the flux of the central object still obscured by intervening material in the homunculus.

TABLE VI

Photometric data of the central object (uncorrected for circumstellar and foreground extinction) adopting different modes for the contribution of the shell entering into D1

\begin{tabular}{lccccccc}
\hline & $V$ & $V-B$ & $\begin{array}{c}B-U \\
(\log \text { intensity) }\end{array}$ & \multicolumn{3}{c}{$\begin{array}{c}U-W \\
(\mathrm{mag})\end{array}$} \\
\hline$\overline{\mathrm{co}}$ (I) & -0.008 & 0.287 & 0.315 & 0.193 & 0.286 & 6.85 & 0.70 \\
$\overline{\mathrm{co}}$ (II) & -0.069 & 0.297 & 0.375 & 0.287 & 0.340 & 7.00 & 0.73 \\
$\overline{\mathrm{co}}$ (III) & -0.067 & 0.265 & 0.265 & 0.136 & 0.255 & 7.00 & 0.65 \\
\hline
\end{tabular}

With the photometric parameters of $(\overline{\mathrm{co}})$ we can now derive those of the homunculus, by subtracing ( $\overline{\mathrm{CO}})$ from the measurements through D1 to D4 in Table IV. The results are denoted shD1, shD2, shD3, and shD4, and are in fact the flux of the shell received through D1, D2, D3, and D4. Note that shD1 is of course the same as the input data to find ( $\overline{\mathrm{co}})$. The results are listed in Table VII for the three different modes of $(\overline{\mathrm{co}})$. From this table and Figure 17 (see Section 7) it is clear that the type of mode does not affect very much the brightness and colours of the entire homunculus (shD4). This is also true for the smaller parts of the homunculus seen through D2 and D3. Notice that the integrated visual brightness of the homunculus (shD4), which is approximately $6^{m} \cdot 3$, is larger than that of $(\overline{\mathrm{co}})$, which is about $7^{m}$ (Table VI). Furthermore, this difference increases towards shorter wavelength, up to $2^{m} .4$ through the $W$ passband (see also below). Thackeray (1953) arrived after measuring the percentage contribution to the intensity of the various zones between the adjacent isophotes of Gaviola (1950), that the nucleus is photographically at least $1^{m} .5$ fainter than the homunculus. This is roughly in agreement with our result.

The decrease of the intensity measured through D4 to D1 will now be further discussed. For this purpose the $V B L U W$ intensities for $(\overline{\mathrm{co}})+(\mathrm{sh})$ listed in Table IV 
TABLE VII

Photometric data of the shell (= homunculus, uncorrected for foreground extinction) as observed through the four diaphragms D1-D4 for three different modes of co (Table VI). The shD1 data were the input data to find $\overline{\text { co }}$ (in Table VI)

\begin{tabular}{lrrrrrrr}
\hline & $V$ & $\begin{array}{c}V-B \\
\text { (log intensity) }\end{array}$ & $\begin{array}{c}B-U \\
\end{array}$ & \multicolumn{7}{c}{$U-W$} & $B-L$ & $V_{\mathbf{J}}$ & $(B-V)_{\mathbf{J}}$ \\
(mag)
\end{tabular}

are plotted against the surface of the diaphragm apertures (denotes $\mathrm{O} 1, \mathrm{O} 2, \mathrm{O} 3$ and $\mathrm{O} 4$ ), expressed in that of D1 (O1) as unit (Figure 13). Corresponding data-points are connected with dashed lines. The curves, fully drawn through the $V$ and $U$ intensities measured through D2, represent the expected decrease of the brightness when it is assumed that each diaphragm receives radiation from an optically thin cylinder homogeneously filled with radiating material. In the determination of the full drawn lines it is assumed, firstly, that the amount of radiation is proportional with the volumes of the cylinders, computed using the measures listed in Table I. Secondly, it is adopted that the thickness of the shell along the line of sight is equal to the diameter of D2, viz. 8."3. It should be noted that D2 has been chosen because it almost covers the visible homunculus along the minor axis (see Figure 11).

Let us now compare the full drawn curves with the dashed curves. The intensities through D4 and D3 are lower than the full drawn curves. This is understandable because these diaphragms cover partly the sky background. If we now go from the data points for $\mathrm{D} 2$ to $\mathrm{D} 1$, the $V$ and $B$ intensities show a decrease with a slope which is more or less the same as that of the full drawn lines. This should actually not be so, since the central object shines through the shell, such as we have seen before (see Section 4.1). However, this coincidence can be explained if the material in the cylinder of D1 is on the average optically thicker than the matter in that of D2. This possibility is strengthened by the fact that there is a strong deficiency of ultraviolet radiation in D1. This effect can be seen in Figure 10, where the D1 measurements are redder than those of D2.

We will now explain how to choose the best value of $(\overline{\mathrm{co}})$, which will be used in the further discussion. The log intensities of $\overline{c o}$ (I) and $\overline{c o}($ II) (Table VI) are plotted close to the zero line in Figure 13. It is evident that those intensities should be higher than 


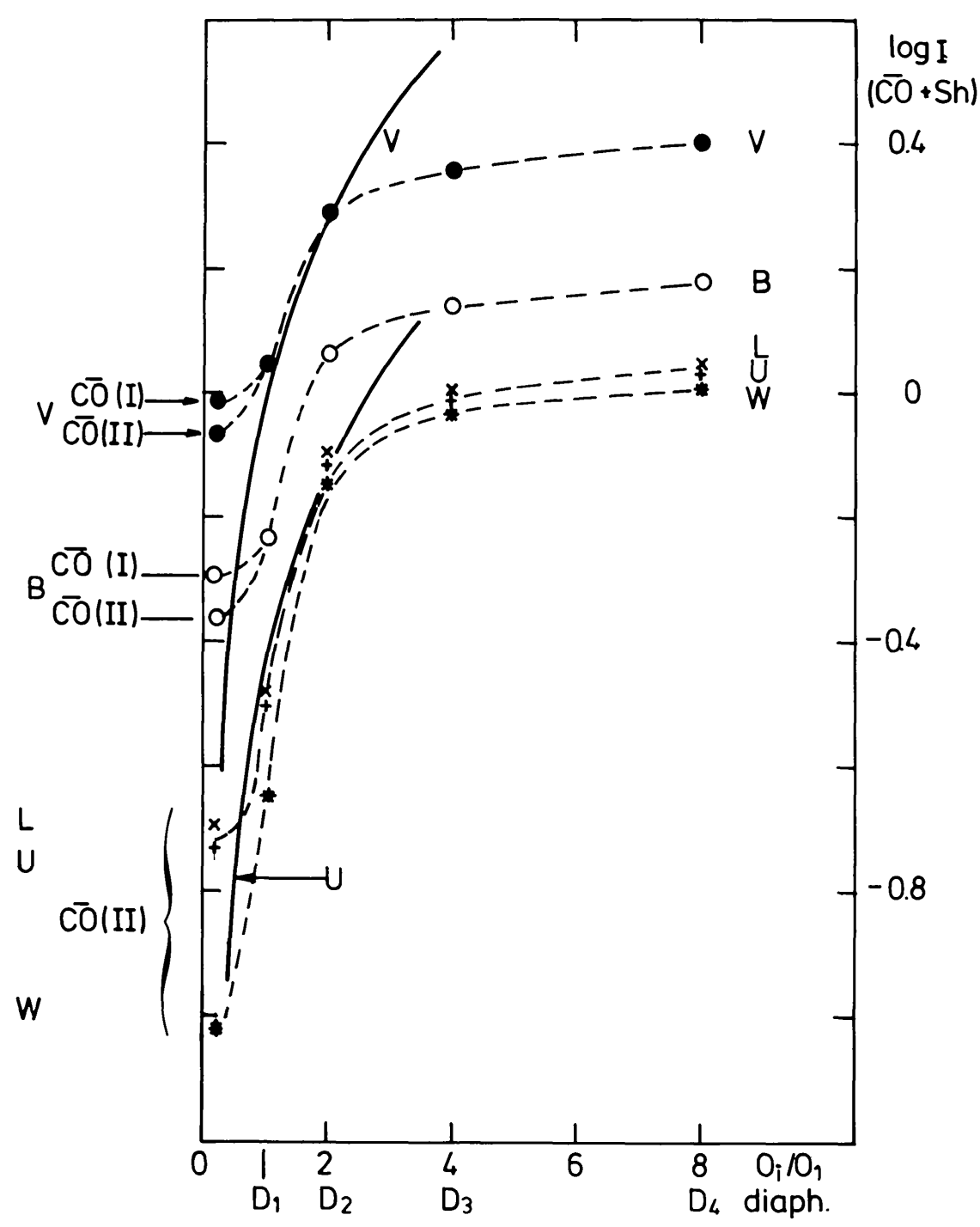

Fig. 13. The luminosity decrease of $\eta$ Car as a function of the diaphragm aperture surface of D1 $\left(=\mathrm{O}_{1}\right)$ as a unit. The estimated brightness for $\overline{c o}$ (I) in $V$ and $B$ and for $\overline{c o}$ (II) (in $V, B, L, U$, and $W$ ) are indicated.

the extension of the full lines connecting D2 and D1, because the central object shines through. Thus at diaphragms smaller than D1, we expect a brightness decline which run more or less like the dotted curves. They should, however, be constantly declining, for example: ( $\overline{\mathrm{co}})$ should not be brighter than the D1 measurements. It is likely that $\overline{\mathrm{co}}(\mathrm{I})$ represents roughly the upper limit for the brightness of $(\overline{\mathrm{co}})$. Note, however, that if $\overline{\mathrm{co}}$ (III) was plotted also, it would lie even higher than $\overline{\mathrm{co}}(\mathrm{I})$. Evidently the lower limit can not be determined, because the full drawn curves approach the zero line asymptotically. From this discussion we adopt $\overline{c o}$ (II) as a reasonable value, which will be employed in our further discussion.

It is of interest to construct a similar diagram as in Figure 13 using the infrared observations of Koornneef (1978) made in April 1974, obtained through five different diaphragms. The result is shown in Figure 14, and will be used to study the photometric 


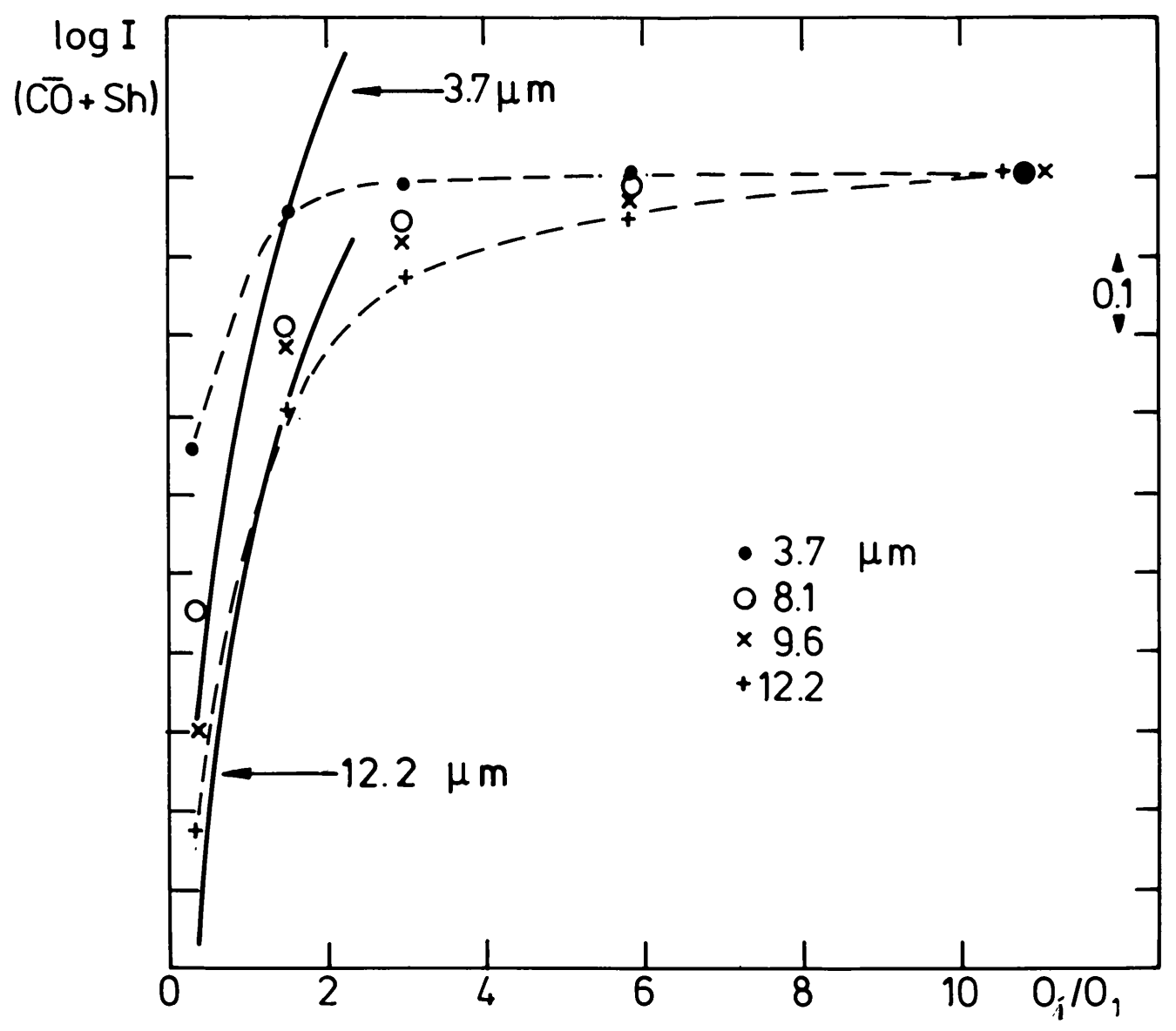

Fig. 14. The same as Figure 13, but now for the infrared observations of Koornneef (1978).

behaviour of the dust shells surrounding $\eta$ Car. The aperture surfaces are expressed in units of the surface of our smallest diaphragm, which we have denoted O1. Koornneef's magnitudes are transformed into log intensities and then normalized to the data of his largest diaphragm. The scale of the vertical axis is taken equal to $0.1 \log$ intensity. The full drawn curves going through the data points of his 7".2 diaphragm measurements for the wavelengths 3.7 and $12.2 \mu \mathrm{m}$, indicate the expected decrease of the brightness, similar to those in Figure 13. For instance, that for the shorter wavelengths the smallest diaphragm measurements have the largest excess radiation with respect to the full drawn curves. Note that because of crowding problems we only indicated the observed decrease of the brightness for 3.7 and $12.2 \mu \mathrm{m}$ (the dashed curves). The above mentioned phenomenon can be expected since Hyland et al. (1979, their Figure 1) found that for shorter infrared wavelengths, the concentration of radiation towards the centre of the homunculus is stronger.

Apparently, dust shells lying closer to the star are warmer, and consequently their thermal radiation peaks more at shorter wavelengths. Note that the existence of one or more dust shells or rings within the central area are proposed by Robinson et al. (1973), Joyce (1975), Hyland et al. (1979), and Warren-Smith et al. (1979). From the central, presumably hot star, no infrared radiation of any importance can be expected. It is 
however possible that the gas envelope situated close around the star redistributes a certain fraction of the stellar energy into the infrared.

\section{The Problem of the Reddening}

There is a wide variety of reddening values of the central object used by different authors. This illustrates the intricacy of the reddening problem (cf. Pottasch et al., 1976; Whitelock et al., 1983). We will discuss this problem below.

Since $\eta$ Car belongs to the open cluster $\operatorname{Tr} 16$, the foreground reddening can be deduced from the photometry of the cluster members. Note that this reddening is partly caused by intracluster matter and partly by the interstellar medium. Feinstein et al. (1973) and Thé etal. (1980a) found an average foreground reddening of $E(B-V)_{\mathrm{J}}=0^{m} .50$ for $\operatorname{Tr} 16$. In Figure 10 the area of the cluster members in the $V-B$ vs $B-U$ diagram as observed by The $e t a l$. is indicated. The width of this area shows the maximum amount of intracluster reddening. The accuracy of the foreground reddening determination is controlled by the width of this strip, which amounts to 0.16 in $V-B$ or $0^{m} .44$ in $(B-V)_{\mathrm{J}}$. Thus any reddening determination has the foreground uncertainty of $\pm 0^{m} \cdot 22$ in $E(B-V)_{\mathrm{J}}$ (but see Section 7).

According to Warren-Smith et al. (1979) $\eta$ Car is surrounded by a dust ring, which attenuates the stellar light (Figure 15). Subsequently the light has to pass through the reflection nebula. Due to scattering in this nebula, the colour of the light beam will be changed. Fore- and background light of the homunculus has been eliminated by our procedure of disentangling the light of the homunculus from that of the central area (see Section 4.2). However, the decrease of the luminosity of (co) as well as the reddening by the material of the homunculus is still present.

The light of $(\overline{c o})$ is certainly not coming directly from the photosphere of the central star, because of the presence of a dense gas envelope close around the hot star (e.g. Craine, 1974). This is suggested by several emission lines in the spectrum of the core of the homunculus. The presence of $P$ Cygni profiles of, for example, permitted and forbidden $\mathrm{Fe}$ II lines indicates that a substantial amount of cool gas is moving outward. The P Cygni profiles of many lines in the ultraviolet spectra of Cassatella et al. (1979) and the same profiles in the high resolution $\mathrm{H} \alpha$-spectrum of Melnick et al. (1982) also prove the presence of outward moving gas. Further evidence of a dense envelope is given by the short exposure photographs ( $\sim 1 \mathrm{~s}$, see Figure 5) of $\eta$ Car by Gaviola (1950) and Gehrz and Ney (1972), which clearly show the central object as a roundish nebula of $\sim 1^{\prime \prime}$ diameter. Nevertheless the star should shine through, otherwise we would not see the emission lines with their P Cygni profiles. Therefore it is extremely difficult to find out what the temperature of the star should be. The same problem was encountered in the case of the two S Dor type stars S Dor and R 71 (= HDE 269006), which possess dense cool envelopes during maximum brightness, dominating the total spectral and photometric appearance of both objects (van Genderen, 1979, 1982; Wolf et al., 1981; Stahl and Wolf, 1982). However, in the case of $\mathrm{R} 71$ it was possible to derive the 


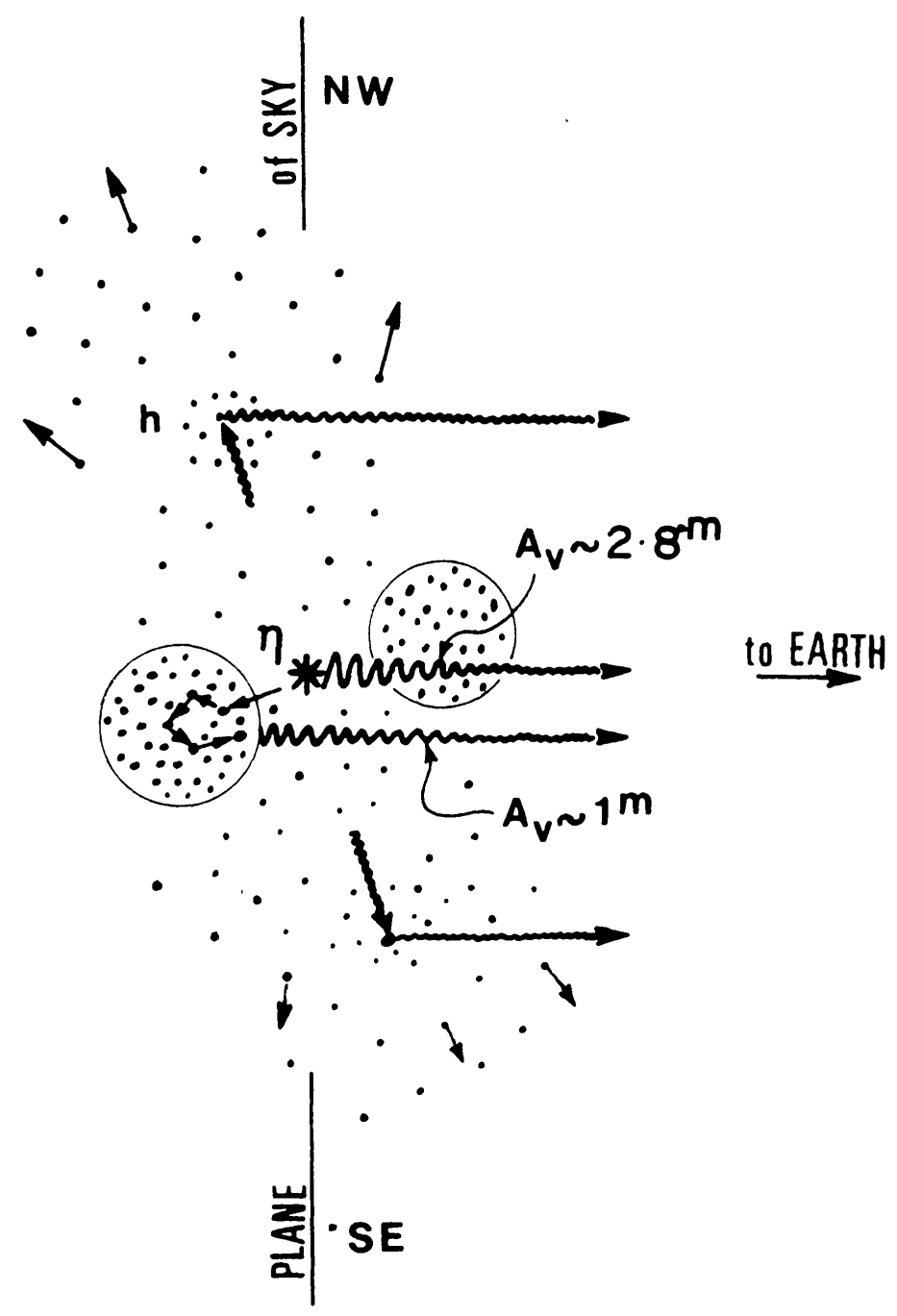

Fig. 15. A cross section through a suggested model of $\eta$ Car proposed by Warren-Smith et al. (1979). The outer part of the homunculus is prolate and inclined at $70^{\circ}$ to the line of sight. Condensation $h$ is expanding from the centre at $20^{\circ}$ angle behind the plane of the sky. The circles are cross sections through the postulated toroidal circumstellar dust ring. (Courtesy Warren-Smith et al., 1979).

temperature of the star photometrically at the time that the envelope was negligible (van Genderen, 1982).

From the discussion above and from those in Sections 6 and 7 it is clear that it will be difficult to obtain a reliable reddening value for the central star. The reasons are the uncertainty in the foreground reddening, the reddening caused by dust in the homunculus and the fact that we do not know the intrinsic characteristics of the star precisely.

\section{The Extinction Law}

It has been explained in Section 5 that it is difficult to determine the value of $E(B-V)_{\mathrm{J}}$. Yet we have to adopt a certain reddening value in order to be able to study $\eta$ Car and its immediate surroundings by means of the two-colour diagrams, and to determine the 
extinction-free spectral energy distribution (Sections 7 and 8). For these studies it is important to define in some detail the extinction law, which will be used.

The ratio of total to selective extinction has been determined with high precision by Barlow and Cohen (1977); they obtained $R=A_{V_{\mathrm{J}}} / E(B-V)_{\mathrm{J}}=3.10 \pm 0.03$. We will adopt this value in our discussions. For the extinction law towards the infrared, actually between $0<1 / \lambda<3$, the Van de Hulst curve No. 15 is generally used in conjunction with the numerical values of $A_{V_{\mathrm{J}}}$ and the colour ratios defined by Johnson (1968). Because Johnson had used $R=3.05$, we have to change his numerical values for $1 / \lambda$ close to zero slightly, in order to agree with the value $R=3.10$.

For ultraviolet wavelengths $\left(3<1 / \lambda<8 \mu \mathrm{m}^{-1}\right)$ we have adopted the extinction law of Savage and Mathis (1979), in such a way that the value of the extinction at the effective wavelength of the Walraven $W$ passband $(0.33 \mu \mathrm{m})$ is equal to that of Savage and Mathis at the same wavelength. The shape of the extinction law defined in this way is smooth and is plotted in Figure 16. Note that in Figure 16 the scale of the vertical

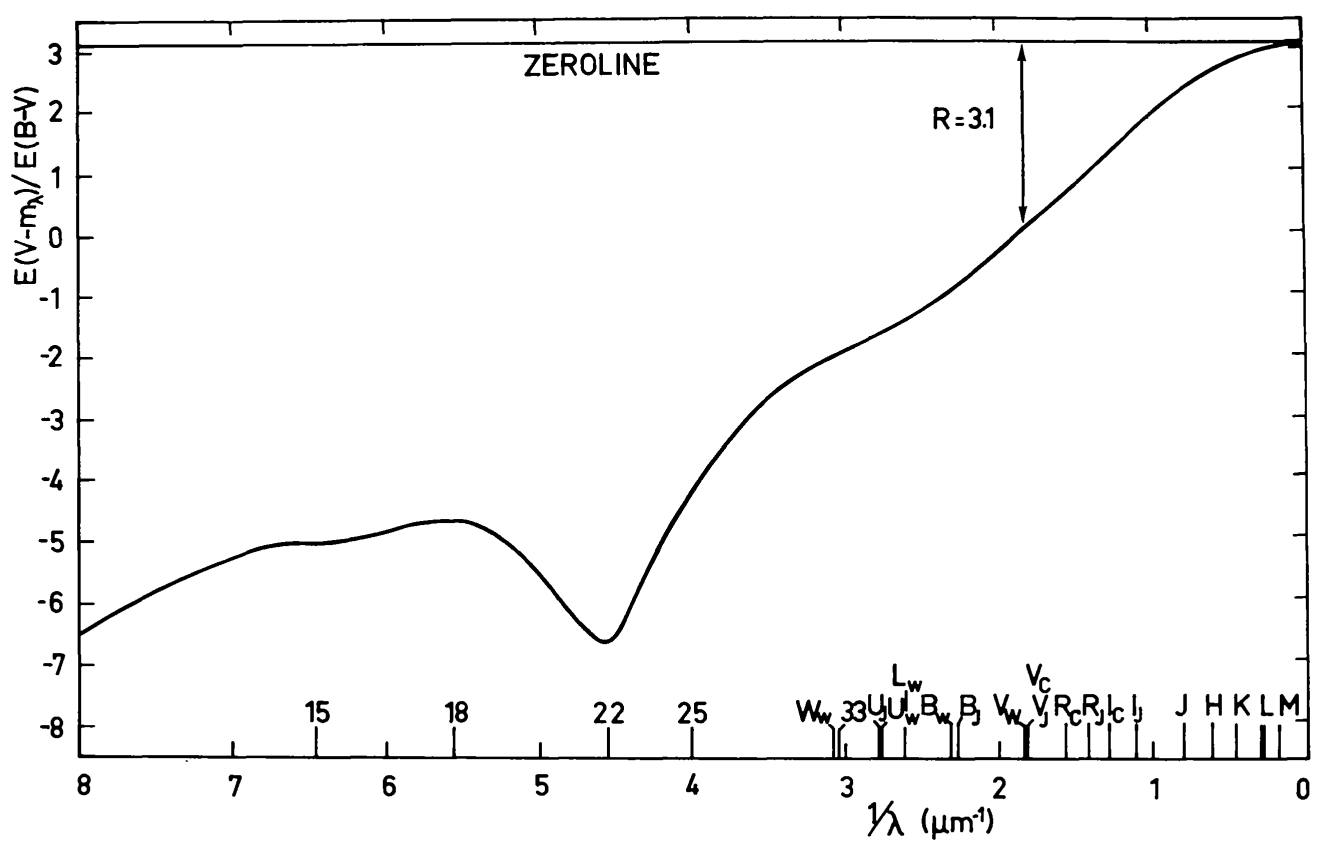

Fig. 16. The extinction law of Savage and Mathis (1979) combined with that of Johnson (1968). Subscripts $\mathrm{C}, \mathrm{J}$, and $\mathrm{W}$ stand for the systems of Cousins, Johnson, and Walraven, respectively.

axis is taken in such a way that $E(B-V)_{\mathrm{J}}=1$. Monochromatic extinctions $A_{\lambda}$ read from this plot are listed in Table VIII expressed in $A_{V_{\mathrm{J}}}$ as unit. It should be noticed that Chlewicki et al. (1984) have shown that there is no universal extinction curve in the ultraviolet and especially in the far ultraviolet. Dereddening with an average curve will therefore most likely lead to large errors. Seven stars near $\eta$ Car also show an extinction curve which deviates from the one of Savage and Mathis. However, since we only go as far as $1 / \lambda\left(\mu \mathrm{m}^{-1}\right) \sim 3$, the errors will be negligible. 
TABLE VIII

Extinction ratios determined using a combined extinction law of Savage and Mathis (1979) and of Johnson (1968)

\begin{tabular}{|c|c|c|}
\hline Passband & $\lambda_{\text {eff }}(\mu \mathrm{m})$ & $A_{\lambda} / A_{V_{\mathrm{J}}}$ \\
\hline \multicolumn{3}{|l|}{ A.N.S. ${ }^{1}$} \\
\hline 15 & 0.1549 & 2.63 \\
\hline 18 & 0.1799 & 2.52 \\
\hline 22 & 0.2200 & 3.12 \\
\hline 25 & 0.2493 & 2.36 \\
\hline 33 & 0.3294 & 1.64 \\
\hline \multicolumn{3}{|l|}{ Walraven $^{2}$} \\
\hline$W$ & 0.3255 & -0.664 \\
\hline$U$ & 0.3633 & -0.616 \\
\hline$L$ & 0.3838 & -0.592 \\
\hline$B$ & 0.4325 & -0.536 \\
\hline$V$ & 0.5467 & -0.408 \\
\hline \multicolumn{3}{|l|}{ Johnson $^{3}$} \\
\hline$U$ & 0.36 & 1.55 \\
\hline$B$ & 0.44 & 1.32 \\
\hline$V$ & 0.55 & 1.00 \\
\hline$R$ & 0.70 & 0.73 \\
\hline$I$ & 0.90 & 0.48 \\
\hline \multicolumn{3}{|l|}{ Cousins $^{4}$} \\
\hline$V$ & 0.55 & 1.00 \\
\hline$R$ & 0.64 & 0.82 \\
\hline$I$ & 0.79 & 0.60 \\
\hline \multicolumn{3}{|c|}{ Koornneef ${ }^{5}$} \\
\hline$J$ & 1.25 & 0.26 \\
\hline$H$ & 1.65 & 0.15 \\
\hline$K$ & 2.2 & 0.10 \\
\hline$L$ & 3.6 & 0.05 \\
\hline$M$ & 4.8 & 0.03 \\
\hline
\end{tabular}

Note that the extinction ratios for the Walraven system are expressed in log Int. units and that they refer to the photometric system of $1970 / 1978$.

References to Table VIII:

1 Wesselius et al. (1982).

${ }^{2}$ Lub and Pel (1977).

3 Johnson (1966).

${ }^{4}$ Bessell (1979).

5 Koornneef (1983).

From the data in Table VIII it is possible to derive colour excess ratios which are important for dereddening purposes in the two-colour diagrams. First of all, note that the Johnson ratio $E(U-B)_{\mathrm{J}} / E(B-V)_{\mathrm{J}}=0.72$ remains unchanged. The different excess ratios in the $(1970 / 1978) \quad V B L U W$ photometric system are as follows: 
$E(B-U) / E(V-B)=0.63, E(B-L) / E(V-B)=0.44, \quad E(U-W) / E(V-B)=0.36$, and $E(B-W) / E(V-B)=1.00$. The values of the first and second excess ratios agree well with that adopted by Lub and Pel (1977). The third one is 0.09 smaller. Also the last ratio is 0.09 smaller than what can be derived from Lub and Pel's ratios. This is the consequence of our demand that the extinction at $0.33 \mu \mathrm{m}$ should coincide with that of Savage and Mathis.

\section{The Colour-Colour Diagrams and the Reddening which Originates in the Homunculus}

Figure 17 shows the three colour-colour diagrams of the central object $(\overline{c o})$. The asterisks indicate the three modes uncorrected for foreground extinction. The plus signs represent the extinction-free modes. For the reddening correction we took $E(B-V)_{\mathrm{J}}=0^{m} .50$, which is equivalent to $E(V-B)=0.195$. Furthermore, using the excess ratios given in Section 6, we can derive $E(B-L)=0.086, E(B-U)=0.123$, and $E(U-W)=0.070$, whereas $A_{V}=3.19 E(V-B)$. Since for our further discussion we will only use the $\overline{\mathrm{co}}$ (II) mode, it is important to visualize its reddening correction and estimated error in Figure 17. They are indicated by the arrow and corresponding error-bar, respectively.

It should be mentioned here that the reddening found by Pottasch et al. (1976) with the aid of the ANS ultraviolet data, viz. $0^{m} \cdot 53 \pm 0^{m} \cdot 06$, is practically equal to the average foreground reddening of $\operatorname{Tr} 16$. Because the brightness of the homunculus is larger than that of ( $\overline{\mathrm{co}}$ ) (by $0^{m} .7$ in $V, 1^{m} .0$ in $B, 1^{m} .7$ in $L$ and $U$ and $2^{m} \cdot 4$ in $W$, deduced from Tables VI and VII) the ultraviolet spectral energy distribution is dominated by the light of the homunculus. Therefore, their procedure of eliminating the $2200 \AA$ bump, is actually finding the foreground reddening of the homunculus, rather than that of the central star. It is thus not surprising that this reddening is practically equal to the one determined by means of the cluster photometry. The same conclusion holds probably for the reddening obtained by Whitelock et al., who derived $E(B-V)_{\mathrm{J}} \sim 0^{m} \cdot 4$ from the ratio of the Paschen $\beta$ and Brackett $\alpha$ line intensities. Thus we are perhaps not far wrong by using $E(B-V)_{J}=0^{m} \cdot 50$, for the foreground extinction.

Let us now examine the position of the central object $\overline{\mathrm{co}}$ (II) in the two-colour diagrams after correction for foreground extinction. If the remaining extinction obeys a normal extinction law for dust, a further reddening correction of $0^{m} \cdot 28+0^{m} \cdot 10$, the net emission in blue light (Section 4.1), in $E(B-V)_{\mathrm{J}}$ is needed (indicated in the lower panel of Figure 17) in order to fit the OBA(PC) relation in the colour-colour diagrams. This is the relation for hot super- and hypergiants having P Cygni profiles in their visual spectrum, meaning that they have mass loss rates exceeding $\dot{M} \sim 2 \times 10^{-6} M_{\odot} \mathrm{yr}^{-1}$ (van Genderen et al., 1983). Note that because of a peculiar deficiency in the $L$ band $\left(\lambda_{\text {eff }} \sim 3840 \AA\right)$ with respect to $U$ and $W, \eta$ Car lies too low in the $B-L$ vs $V-B$ diagram (see also Figure 18). If the above shift in the other two colour-colour diagrams to the OBA(PC) relation is correct, the temperature of the star will not be more than $10000 \mathrm{~K}$. In view of the evidence presented before that the star presumably is surround- 


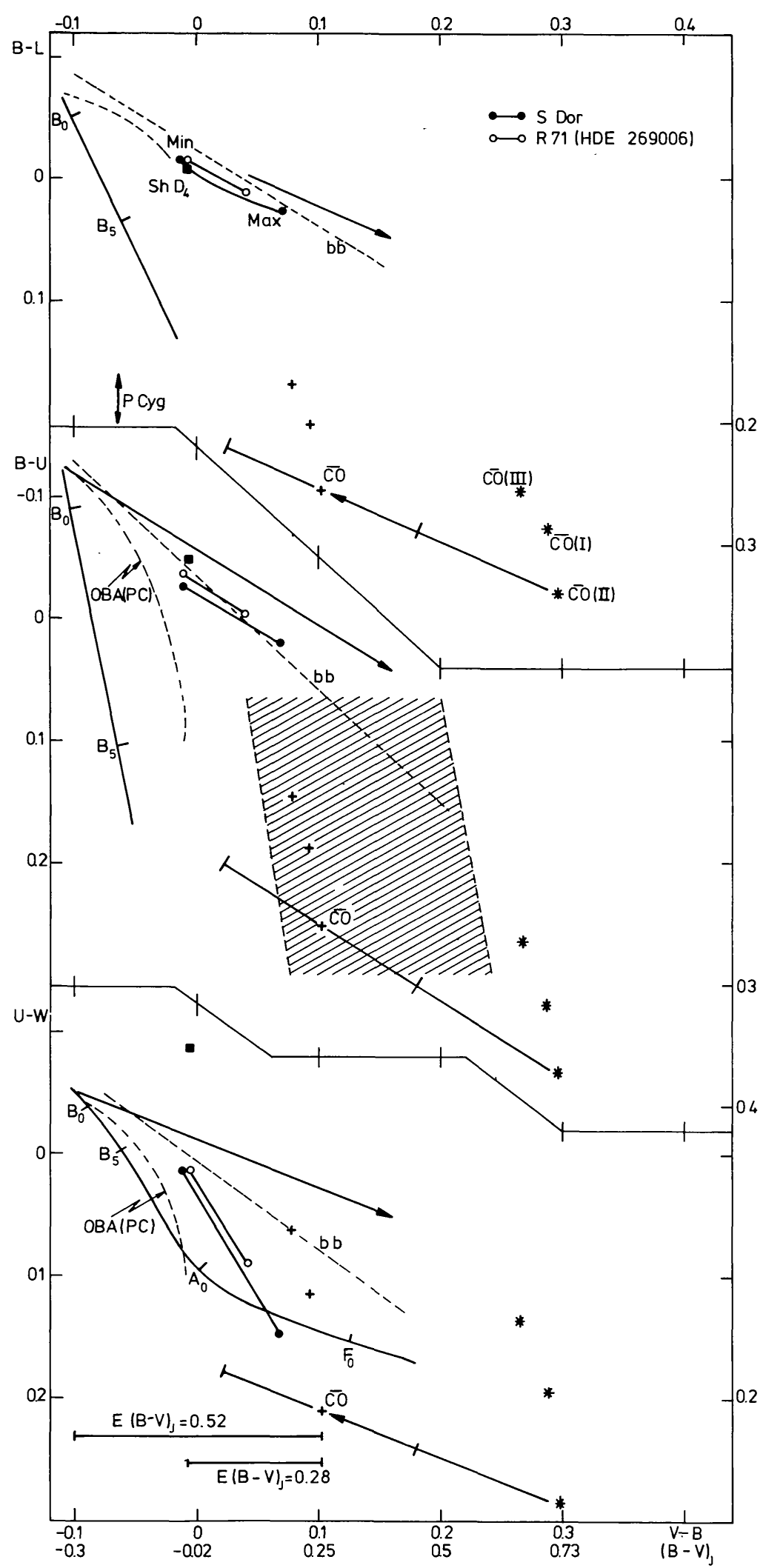

Fig. 17. The two-colour diagrams with observed and dereddened colours of the central object of $\eta$ Car corrected for foreground reddening, but not corrected for the reddening by the intervening material in the homunculus $(\overline{\mathrm{co}})$. Star-like symbols: three modes for the central object, uncorrected for foreground reddening. Plus signs: the same but now corrected for the foreground reddening $E(B-V)_{\mathrm{J}}=0^{m}$.50. Black square: the homunculus (without central object) corrected for the foreground reddening $E(B-V)_{\mathrm{J}}=0.50$. 
ed by a relative cool gas envelope and because of astrophysical reasons, the temperature of the star must be much higher. For instance, the presence of emission lines like Fe III, N III, and Ne III ionized by stellar far ultraviolet photons in a dense compact H II region, which is probably the circumstellar gas envelope (Andriesse et al., 1978), and the strength of $\mathrm{H} \beta$ (Davidson, 1971), indicate that a temperature of $\sim 30000 \mathrm{~K}$ would fit these facts much better (see also Craine, 1974). In this connection it is important to mention that from recent studies of the X-ray emission in the direction of $\eta$ Car, Chlebowski et al. (1984) concludes, that the characteristics of the stellar wind are typical for a massive hot star.

Let us thus assume that the central star intrinsically must be located somewhere at the top of the OBA (PC) line, close to the point where it intersects the main sequence. In this region of the two-colour diagram temperatures range from 30000 to $50000 \mathrm{~K}$. Then the maximum reddening caused by circumstellar material (gas in the circumstellar shell, see further, and dust in the homunculus) is $E(B-V)_{\mathbf{J}}=0.52+0 m \cdot 10=0.62$, in which the $0^{m} .10$ is caused by the net emission in blue light (see Section 4.1). This value is lower than adopted or suggested by, for example, Hyland et al. (1'm3) and WarrenSmith et al. (0m.9), but somewhat closer to that of Andriesse et al. (0m.7). Note that part of this $0^{m} .62$ must be caused by dust alone. It is important to mention here that the reddening mechanism by cooler gas surrounding a hot star is photometrically detected at the stars S Dor and R 71 (= HDE 269006) in the LMC. Both stars were observed in the $V B L U W$ system during several years. The colour variation from minimum light (gas envelope at low density) to maximum light (gas envelope at high density) is schematically indicated in Figure 17 (see van Genderen, 1982). The reddening effect is visible in all colour indices and the visual brightening of the objects by 1 to 2 mag is due to redistribution of ultraviolet radiation into the visual by the cooler envelope (see also Section 9.2). It is important to note here that the reddening by the gas in the $V-B$ vs $B-L$ and $V-B$ vs $B-U$ diagrams is only accidentally more or less parallel to the reddening line for the dust. This conclusion is drawn from the fact that the infrared excess is low (Glass, 1974; Allen and Glass, 1976), because there is no dust present in the envelope of S Dor. Recently Glass (1984) has detected a dust shell around R 71, but it is unlikely that the reddening during an outburst (visible as a strong visual brightness increase) is caused by a growing dust shell. He believes that the dust shell is probably a permanent feature of $\mathrm{R} 71$. Indeed, the gas envelope at maximum light has temperatures of the order of $10000 \mathrm{~K}$, while at minimum light the star, which can be seen undisturbed, appears to have temperatures of the order of $14000 \mathrm{~K}$ (Wolf et al., 1981; van Genderen, 1982; Stahl and Wolf, 1982).

We conclude that the central star of $\eta$ Car is now also suffering from a so called ' $\mathrm{S}$ Dor effect' (see also Section 9.2). A dense envelope has built up close around the star by its wind an by a few small outbursts since 1870, which is able to change the spectral energy distribution. This new envelope of $\eta$ Car is presumably of a much larger size and a higher density and temperature than those of S Dor and R 71 in maximum state. We therefore do not expect the same type of reddening or redistribution process. To move $\overline{\mathrm{co}}$ (II) up to its presumed intrinsic position, the slopes of the reddening lines should be 
very steep indeed, especially considering the fact that still part of it will consists of dust reddening originating in the homunculus.

An estimate of the average value of $R$ valid for the combined extinction by the gas shell surrounding the star and the dust in the homunculus, can be made as follows. The visual brightness of the central extinction $V_{\mathrm{J}, 0}\left(\overline{\mathrm{CO}}(\mathrm{II}) \equiv V_{\mathrm{J}, 0}(\overline{\mathrm{co}})=5^{m} \cdot 45\right.$ (from now on we omit the mode number II). With a distance of $2800 \mathrm{pc}$ (e.g. Thé et al., 1980a) we obtain $M_{V_{J}}(\overline{\mathrm{co}})=-6^{m} .8$. The total luminosity of $\eta$ Car and thus also from the central star $(s)$, derived from the observed spectral energy distribution (see Section 8) $\log L / L_{\odot}=6.82$. Thus $M_{\text {bol }}(s)=-12^{m} .3$, with $T_{\text {eff }} \sim 30000 \mathrm{~K}, B C \sim-2^{m} .75$ and consequently $M_{V_{\mathrm{J}}}(s) \sim-9 \mathrm{~m} .5$. Therefore the visual brightness of $(\overline{\mathrm{co}})$ shows a deficiency of $\sim 2^{m} .7$, with respect to that of $(s)$. This means that $\sim 90 \%$ of the visual energy disappeared, partly by redistribution of energy by the gas shell and partly by extinction of the dust particles in the homunculus. We tentatively assume that the gas shell caused a decrease of light, which lies between $2^{m} .7$ and $0^{m} .8$ (because $A_{V_{J}}(\mathrm{dust})<1^{m} \cdot 9$, see further) and much more in the ultraviolet, while in the case of S Dor and R 71 the gas shells caused an increase of light of $1^{m}$ to $2^{m}$. Thus the average extinction $\left\langle A_{V_{\mathrm{J}}}\right\rangle \sim 2^{m} .7$ combined with $\left\langle E(B-V)_{\mathrm{J}}\right\rangle \sim 0^{m} .62$, the value of $\langle R\rangle \sim 4.4$. Note that if there was no correction for a net line emission in the blue amounting to 0 . 10 then $\langle R\rangle \sim 5.2$. Andriesse et al. (1978), using other arguments, adopted $R=5.4 \pm 1.5$. It is difficult to say what the individual values for $R$ should be. According to Thé $e t$ al. (1980b) and Thé and Groot (1983), the extinction law towards O-type stars embedded in the Carina region, is anomalous with $R$ ranging from 3.1 to 4.4 . This is presumably caused by a larger than normal size distribution of the dust particles in the environment of the $O$ stars (Herbst, 1976; Chini and Krügel, 1983). However, in the homunculus, polarization and scattering properties of the dust particles indicate smaller sizes of about $0.1 \mu \mathrm{m}$ (Warren-Smith et al., 1979), compared to that of dust particles in the general interstellar medium, which have sizes of $0.15 \mu \mathrm{m}$, according to Greenberg (1966). The very blue colour of the homunculus also suggests small particles, perhaps even smaller than $0.1 \mu \mathrm{m}$. Thus we do not expect for the dust particles in the homunculus an extremely high value of $R$. Consequently, the high value for $\langle R\rangle$ just calculated might be largely caused by the attenuation of the stellar light by the surrounding cooler gas shell. This is supported by the anomalous ratios for $E(B-L) / E(V-B), E(B-U) / E(V-B)$, and $E(U-W) / E(V-B)$, which are all larger than 1 . Note that the $R$ values for $\mathrm{S}$ Dor and R 71 are negative because of the opposite behaviour of brightness and colour during the outbursts. We must conclude that the reddening by dust alone in the homunculus should be $E(B-V)_{\mathrm{J}}<0^{m} .62$ or $A_{V_{\mathrm{J}}}<1^{m}$.9. This is very low compared with that for the bi-polar nebulae, where sometimes the central star is attenuated by more than $20^{m}$ (Neckel and Staude, 1984). According to the ratio of maximum polarization to colour excess of Serkowski et al. (1975), the degree of polarization in front of the central object should be $<5 \%$ if $E(B-V)_{\mathrm{J}}<0^{m} .62$. This is indeed not in contradiction with the polarization measurements of Warren-Smith et al., if their Figure 2 is extrapolated to the centre of the nebula.

The dust model for the homunculus proposed by Mitchell and Robinson (1978) 
reveals an extinction of at least twice as high as our value. This will be explained below. According to their best model (No. 3) the total mass of the dust particles amounts to $M_{d}=0.03 M_{\odot}$, the weighted average density of the grains $\langle s\rangle=7.1 \mathrm{~g} \mathrm{~cm}^{-3}$ and the grain radius $a=0.1 \mu \mathrm{m}$. The total number of grains per unit of volume

$$
n_{d}=\frac{\text { mass per unit of volume }}{\text { mass of dust grain }}=\frac{M_{d}}{V} \frac{1}{v\langle s\rangle}
$$

with $V$ the volume of the homunculus with radius $R=0.1 \mathrm{pc}$ and $v$ the volume of a dust grain. The visual extinction $A_{V_{\mathrm{J}}}=1.086 \tau$, in which $\tau$ is the optical depth:

$$
\tau=Q n_{d} R \Pi a^{2}
$$

In this formula $Q=$ blocking area $/ \Pi a^{2}$ is the extinction efficiency; it is of the order of 2. The result is $A_{V_{\mathrm{J}}}=3^{m}$.7, which is more than twice our upper limit.

It is possible to show that our upper limit of $A_{V_{\mathrm{J}}}=1^{m}$.9 should perhaps be still lower. Let us consider Figure 17 once more. The position for the light of the homunculus (shD4 mode II) is indicated by the black square (data of Table VII corrected for foreground reddening; note that there is not much difference between the three modes). In the $V-B$ vs $B-L$ and $V-B$ vs $B-U$ diagrams this light mimics a supergiant with $T_{\text {eff }} \sim 20000 \mathrm{~K}$. In the $V-B$ vs $U-W$ diagram, $U-W$ shows an excess in $W$ ( $\left.\lambda_{\text {eff }} \sim 3250 \AA\right)$. Thus shD4 is bluer in all colour indices compared to ( $\left.\overline{\mathrm{co}}\right)$. The facts, that $\eta$ Car has an elliptical shape, that it shows optical polarization consistent with reflections and that there is a direct evidence for a disk oriented in the expected direction, makes it look like the bi-polar reflection nebulae, although the dust properties are completely different. In these nebulae, scattering by small particles cause a net bluing to the spectrum, while their absorption spectrum is more or less similar to that of the central star (Schmidt et al., 1978). The same may be more or less the case for $\eta$ Car, with the difference that the light of the homunculus contains extra emission lines from the gas envelope around the star and the condensations. Thus at the risk of overinterpretation of these data, we may conclude that shD4 represents roughly the scattered light of (co). Therefore it can be expected that (co) is redder than shD4 in all colour indices. The difference in $(B-V)_{\mathrm{J}}$ between $(\overline{\mathrm{co}})$ and shD4 is $\sim 0.28$ (the range is indicated at the bottom of Figure 17), thus the difference between (co) and shD4 should be $<0.28$ or $A_{V_{\mathrm{J}}}<0.9$. The difference between the dust model of Mitchell and Robinson is now at least a factor of four. One should realize that the reddening suffered by (co) is not necessarily representative for the whole homunculus, because its dusty material may not be uniformly distributed (see Section 4.1). Consequently our low extinction does not disprove the dust model of Mitchell and Robinson. We fear that no better determination of the extinction of the dust in the homunculus is possible at present.

\section{The Spectral Energy Distribution and the Total Luminosity}

Figure 18 shows the observed spectral energy distribution $\log F_{\lambda}^{\prime}\left(\mathrm{W} \mathrm{m}^{-2} \mu \mathrm{m}^{-1}\right)$ plotted against $\log \lambda(\mu \mathrm{m})$ for the spectral range $0.15<\lambda<175 \mu \mathrm{m}$, based on observations from 


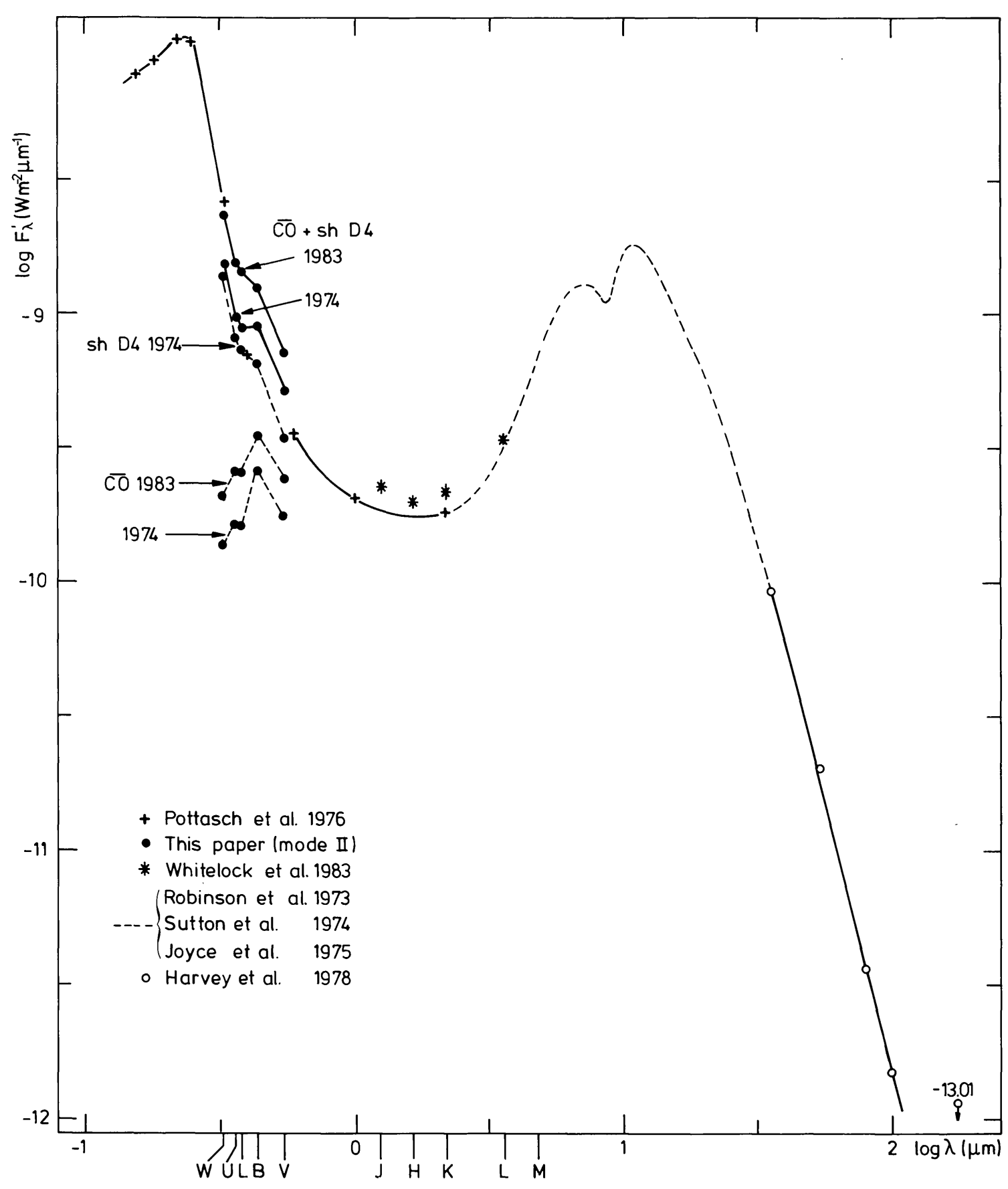

Fig. 18. The observed spectral energy distribution $\log F_{\lambda}^{\prime}\left(\mathrm{W} \mathrm{m}^{-2} \mu \mathrm{m}^{-1}\right)$ against $\log \lambda(\mu \mathrm{m})$ corrected for foreground reddening of $\eta \mathrm{Car}$ from $0.15<\lambda<175 \mu \mathrm{m}$ based on the results of various sources (see also Section 8). A differentiation is made between the contributions of the central object still attenuated by the intervening material in the homunculus ( $\overline{\mathrm{co}})$, the shell (shD4) and the sum of both $(\overline{\mathrm{co}}+\operatorname{shD} 4)$.

various sources. Only a few of the individual observations were plotted. The best coverage of data has been obtained in the early seventies. The mean curve used for the determination of the total flux is therefore also mainly based on these observations, except those with $\log \lambda>1.5$, which were made in 1977 by Harvey et al. (1978). A foreground reddening correction of $E(B-V)_{\mathrm{J}}=0^{m} .50$ has been applied for the shorter 
wavelengths $\lambda<5 \mu \mathrm{m}$, using the extinction law based on $R=3.1$. It appears that the values listed by Pottasch et al. (1973, their Table III) for $\lambda=19.5$ and $34 \mu \mathrm{m}$ were higher by 0.1 to 0.3 in $\log F_{\lambda}^{\prime}$ than actually observed by Gehrz et al. (1973), Robinson et al. (1973), and Sutton et al. (1974). The later infrared observations of Harvey et al. (1978) covering the range $\lambda=35$ to $175 \mu \mathrm{m}$, appear to support that the curve should run much steeper than that indicated by these two values of Pottasch et al. The reason for this inconsistency is unknown. Therefore, we will ignore these two values.

We have further plotted the corrected flux densities (for mode II) based on the $V B L U W$ observations for the entire object $(\overline{c o}+\operatorname{shD} 4)$ for the years 1974 and 1983 , for the homunculus (shD4), only the 1974 data to prevent confusion, and for the central object still influenced by the dust in the homunculus ( $\overline{\mathrm{co}})$, also for 1974 and 1983 . They are listed in Table IX in which mode I is only listed for June 1983. There is a satisfactory agreement with the flux densities of Pottasch et al. at $\lambda=0.33 \mu \mathrm{m}$ and received in the $W$ passband at $\lambda=0.325 \mu \mathrm{m}$. The large deficiency in the ultraviolet of $(\overline{\mathrm{co}})$ is peculiar (Section 4.2) and cannot be explained by the extinction of dust alone. Perhaps gas in the homunculus or in the circumstellar envelope absorbs energy in the Balmer lines and Balmer continuum.

TABLE IX

The energy distribution in the $V B L U W$ system of the different components of $\eta$ Car, using a foreground reddening of $E(B-V)_{\mathrm{J}}=0.50$

\begin{tabular}{|c|c|c|c|c|c|c|c|c|}
\hline \multirow{2}{*}{$\begin{array}{l}\lambda \\
(\AA)\end{array}$} & \multirow{2}{*}{$\begin{array}{l}\log \lambda \\
(\mu \mathrm{m})\end{array}$} & \multirow[b]{2}{*}{ Band } & \multirow[b]{2}{*}{ Date } & \multicolumn{5}{|c|}{ 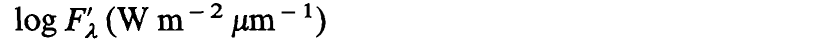 } \\
\hline & & & & $\overline{\mathrm{co}}+\operatorname{shD} 4$ & $\operatorname{shD} 4(\mathrm{I})$ & $\operatorname{shD} 4(\mathrm{II})$ & $\overline{\mathrm{co}}(\mathrm{I})$ & $\overline{\mathrm{co}}(\mathrm{II})$ \\
\hline \multirow[t]{2}{*}{5467} & -0.262 & $V$ & April 1974 & -9.282 & & -9.462 & & -9.751 \\
\hline & & & June 1983 & -9.142 & -9.357 & -9.322 & -9.550 & -9.611 \\
\hline \multirow[t]{2}{*}{4325} & -0.364 & $B$ & April 1974 & -9.043 & & -9.189 & & -9.587 \\
\hline & & & June 1983 & -8.903 & -9.081 & -9.049 & -9.376 & -9.447 \\
\hline \multirow[t]{2}{*}{3838} & -0.416 & $L$ & April 1974 & -9.050 & & -9.135 & & -9.798 \\
\hline & & & June 1983 & -8.846 & -8.964 & -8.931 & -9.469 & -9.594 \\
\hline \multirow[t]{2}{*}{3633} & -0.440 & $U$ & April 1974 & -9.010 & & -9.089 & & -9.788 \\
\hline & & & June 1983 & -8.810 & -8.921 & -8.889 & -9.457 & -9.588 \\
\hline \multirow[t]{2}{*}{3255} & -0.487 & $W$ & April 1974 & -8.818 & & -8.859 & & -9.862 \\
\hline & & & June 1983 & -8.638 & -8.709 & -8.679 & -9.457 & -9.682 \\
\hline
\end{tabular}

There are two major radiation peaks, one in the ultraviolet and one in the infrared. The latter consists of two separate maxima (e.g. Robinson et al., 1973; Joyce, 1975). The light of the homunculus is obviously dominant in the optical region, while that of $(\overline{\mathrm{co}})$ is of lower luminosity with a large deficiency in the ultraviolet passbands $L, U$, and $W$ as discussed before (see also its low position in Figure 17).

A minimum is reached in the $J H K L$ passbands. A detailed spectrum in this region made in early 1981 by Whitelock et al. (1983) reveals several emission features. Their absolute fluxes were transformed to $\log F_{\lambda}^{\prime}$ with the aid of the calibration of Strecker 
et al. (1979) of $\alpha \mathrm{CMa}$ and the JHKL magnitudes observed on the same dates as the spectrum by Whitelock et al. After applying a reddening correction it appears that the flux at this wavelength region is also higher in 1981 than in the early seventies, similar to the region at shorter wavelengths.

The far infrared flux observed by Harvey et al. (1978) runs steeper than in the portion just at longer wavelength than the double maximum. Preliminary IRAS results at 50 and $100 \mu \mathrm{m}$ seem to indicate even lower fluxes than these (Olnon, 1984).

The total flux for the observed curve between $0.15<\lambda<175 \mu \mathrm{m}$ amounts to $26 \times 10^{-9} \mathrm{~W} \mathrm{~m}^{-2}$. Adding to this value the unobserved flux for $\log \lambda<-0.8$ and all emission features, we estimate a total flux (corrected for $E(B-V)_{\mathrm{J}}=0^{m} .50$ ): $F^{\prime}=(26.5 \pm 2) \times 10^{-9} \mathrm{~W} \mathrm{~m}^{-2}$. Thus the luminosity for the central star should be $L(s)=4 \Pi D^{2} F^{\prime}$ and with $D=2800 \pm 200 \mathrm{pc}$, we obtain: $L(s)=(6.61 \pm 1.02) \times 10^{6} L_{\odot}$ or $\log L(s) / L_{\odot}=6.82 \pm 0.07$ and $M_{\text {bol }}(s)=-12^{m} .3 \pm 0^{m} .2$. This value indicates a somewhat greater luminosity than usually assumed in the literature, viz. $M_{\text {bol }}=-12^{m} .0$.

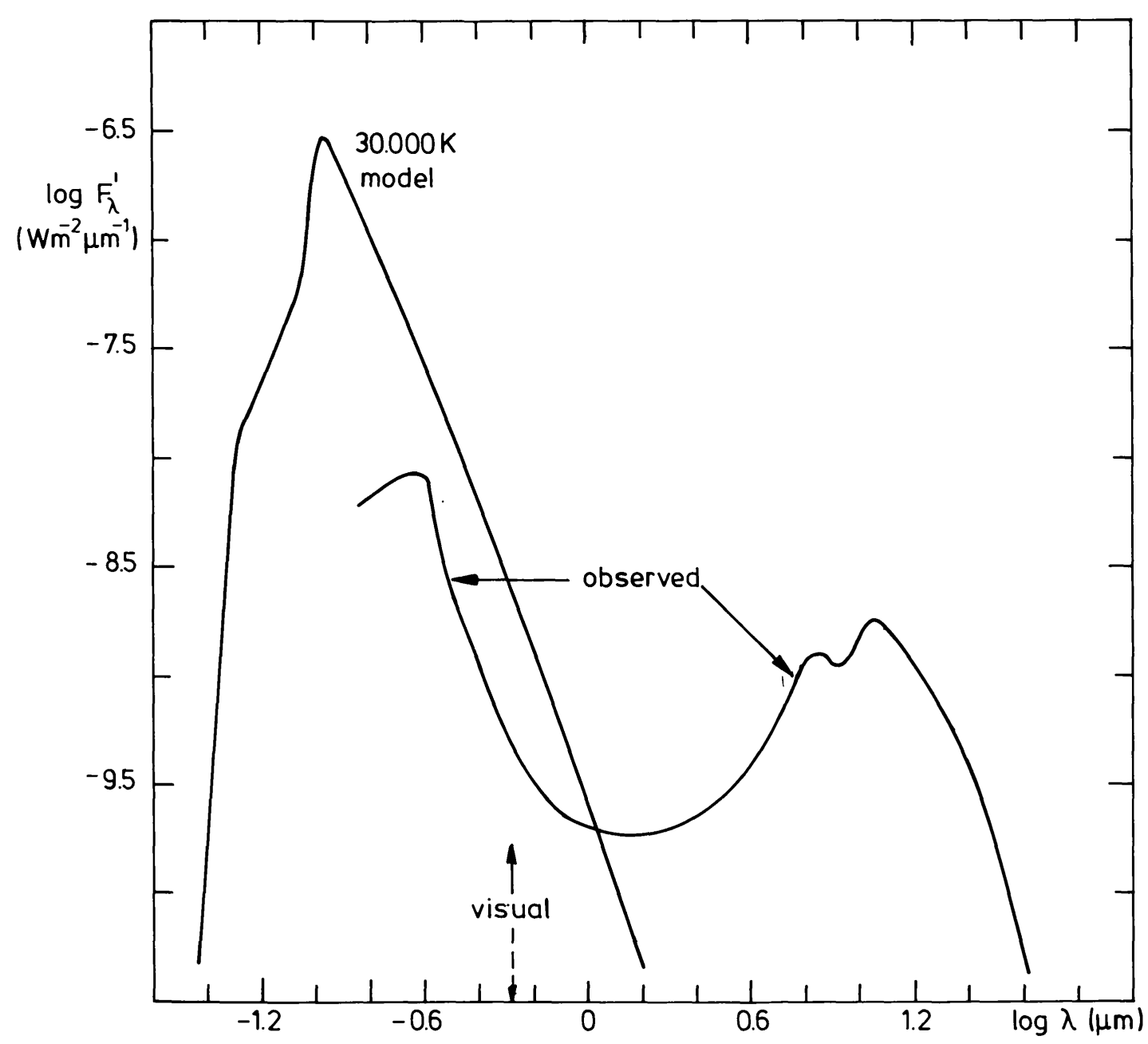

Fig. 19. A comparison of the observed spectral energy distribution corrected for foreground reddening of $\eta$ Car and of a $30000 \mathrm{~K}$ model of Kurucz (1979) with $\log g=3.5$, placed at the same distance as $\eta$ Car (2800 pc). 
The total luminosity just found can be expressed in terms of a temperature and a radius. With $T_{\text {eff }}=25000,30000$, or $35000 \mathrm{~K}$, the radius is $R=138,96$, and $70 R_{\odot}$, respectively.

Figure 19 shows schematically the observed spectral energy distributions $\log F_{\lambda}^{\prime}$ $\left(\mathrm{W} \mathrm{m}^{-2} \mu \mathrm{m}^{-1}\right.$ ) against $\log \lambda(\mu \mathrm{m})$ and the theoretical Kurucz model for $T_{\text {eff }}=30000 \mathrm{~K}$ and $\log g=3.5$ (Kurucz, 1979). The diagram shows the dramatic redistribution of the ultraviolet flux of the central star into the infrared, mainly via thermal radiation of the homunculus. Only $\sim 8 \%$ of the total radiation is received in the optical region, while $\sim 92 \%$ is received in the infrared. The visual radiation is only a small fraction of the optical region.

The flux density at $1415 \mathrm{MHz}(\lambda=21 \mathrm{~cm})$ amounts to $0.9 \mathrm{Jy}$ or $6 \times 10^{-23} \mathrm{~W} \mathrm{~m}^{-2} \mu \mathrm{m}^{-1}$ (Retallack, 1983), which is very low compared with, for example, the flux density in the visual region, viz. $\sim 5 \times 10^{-10} \mathrm{~W} \mathrm{~m}^{-2} \mu \mathrm{m}^{-1}$. The observed X-ray emission of $\eta$ Car amounts to only $10^{-6}$ of the total luminosity (Chlebowski et al., 1984). The flux density appears to be $\sim 50$ times lower than in the visual region.

So far we assumed that $\eta$ Car consists of one single star only. Assume that we are dealing with a double or a triple star. The primary (we assume that it is responsible for the outbursts) has a minimum value if all components of the system are of equal luminosity. The corresponding minimum values for $\log L / L_{\odot}$ are indicated in Figure 22 $\left(M_{\mathrm{bol}}=-11^{m} .55\right.$ and $-11^{m} .11$, respectively). For $T_{\text {eff }}=30000 \mathrm{~K}$, the minimum radii are then 68.5 and $56 R_{\odot}$, respectively, and the total fluxes $F^{\prime}$ subsequently a factor 2 and 3 smaller than in the case of a single star. Nevertheless, even in these extreme cases the primary is still one of the brightest stars known.

\section{A Rediscussion of the Past and Present Behaviour of $\boldsymbol{\eta}$ Car and a Comparison with P Cyg and Other S Dor Type Stars. The Past and Present Mass Loss Rates}

\subsection{THE PHOTOMETRIC HISTORY OF $\eta$ Car}

Figure 20 shows the photometric history of $\eta$ Car (in old astronomical literature also called $\eta$ Argus) and adapted from the compilations of Innes (1903) and Gratton (1963): both from historical times up to AD 1900, of O'Connell (1956): the interval 1935-1952, of Feinstein and Marraco (1974): the interval 1963-1973, of the visual observers Jones and Bateson (see Bateson 1952-1970): the interval 1952-1970 and finally of this paper: the interval 1973-1983. De Vaucouleurs and Eggen (1952) obtained a number of photoelectric observations in 1952. Notice that the scale for the interval 1900-2000 is twice larger than that for the previous centuries. Numbers between brackets (added to the light curve) refer to the references in the caption of Figure 20.

Those of O'Connell are photographic magnitudes and are therefore roughly transformed to visual magnitudes by subtracting from $m_{p g} 0$. 8 . We have found this correction in the following way. Based on three photographic magnitudes given by Innes and on visual estimates (Jones and Bateson) made at roughly the same time, we found a 


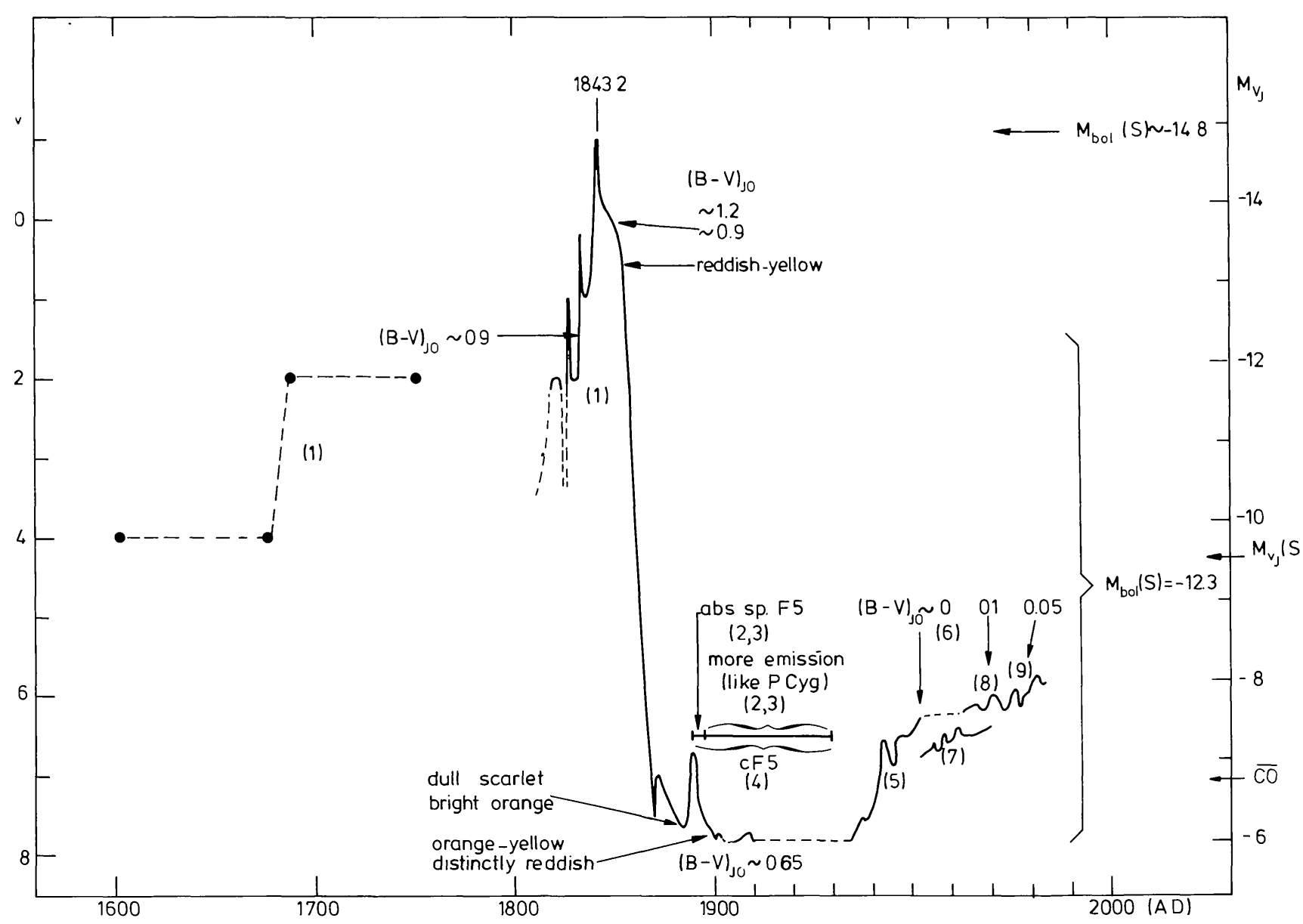

Fig. 20. The photometric history of $\eta$ Car. Bracketed numbers refer to the following references: (1) Innes (1903) and Gratton (1963); (2) Cannon (1897); (3) Cannon (1916); (4) Whitney (1952); (5) O'Connell (1956); (6) de Vaucouleurs and Eggen (1952) give $C p=0.42$, this is equivalent to $(B-V)_{\mathbf{J}}=0.49$ if the formulae given by Allen (1955) are used; (7) Bateson (1952-1970); (8) Feinstein and Marraco (1974); (9) this paper. Note that the scale for the time interval 1900 to 2000 is twice as large as that for the other centuries.

difference of $\sim 1^{m}$. 2 . However, adopting that the magnitude scale of the visual observers Jones and Bateson is consistent from 1952 until 1970, and considering the systematic difference of these estimates with the corrected magnitudes of O'Connell and of the $V_{\mathbf{J}}$ magnitudes of Feinstein and Marraco and of this paper, the correction should be 0.8 . We are however aware that the uncertainty in this correction is large and it is certainly not a constant in view of the fact that the colour became bluer by $\sim 0^{m} .6$ from 1930 to 1940, while the photographic magnitude rose by $\sim 1^{m} .5$ (Section 9.2). Thus the rise in the visual was presumably not more than $\sim 0^{m} .9$. The fact that the magnitude scale of the visual observers differ systematically with the $V_{\mathrm{J}}$ scale by $\sim 0^{m} .4$ illustrates the difficulty of estimating the integrated magnitudes of an extended object.

In order to find an interpretation of the light variation, the behaviour of the colour index $(B-V)_{\mathrm{J}}$ is of importance. Therefore, we examined the scarce announcements of the early observers on the colour. Usually they compared the colour of $\eta$ Car with well-known late type bright stars like $\alpha$ Cen (G0-K5), Arcturus (K0), Aldebaran (K5), 
etc. The colour index $(B-V)_{\mathrm{J}}$ is then estimated and corrected for the foreground reddening $E(B-V)_{\mathrm{J}}=0 m 50$. The derived values for the intrinsic colour of $\eta$ Car are indicated next to the light curve. It appears that during the high maximum, lasting $\sim 30 \mathrm{yr}$ (from $\sim 1830$ to 1860 ), the intrinsic colour was presumably $(B-V)_{\mathrm{J}, 0} \sim 0^{m} .9$. In the following deep minimum the description of colour varies from dull scarlet and distinctly reddish to bright orange and orange-yellow (1880-1895). On the average it seems to be less red. During that time, the first spectra were made, showing an absorption spectrum of spectral type F5 and having characteristics like that of P Cyg (Cannon, 1897, 1916). Later Whitney (1952) classified these spectra as that of an F-type supergiant ( $\mathrm{cF} 5)$. Although we do not believe that the central star was really an F-type, but rather an early B-type star with an extended dense cooler gas shell, which simulated the F-type absorption spectrum, we presume that the intrinsic colour of that combination was somewhat bluer than at maximum even with the presence of dust in the young homunculus. For example if $(B-V)_{\mathrm{J}, 0}$ was $\sim 0^{m} .65$ (Section 9.2) and if we add the foreground reddening to this, it would certainly have made the impression of orangeyellow (Innes, 1903) and bright orange (Thorne, see Innes, 1903). The other descriptions of 'dull scarlet' and 'distinctly reddish' by some others seem to indicate a redder colour. However, real colour variations are not excluded in view of the small bursts still present at minimum light. Anyway, the absorption spectrum at minimum was quickly replaced by more and more emission lines (Cannon, 1916) and still shows a wealth of emission features (Aller, 1970). The photographic brightness rose quickly by $1^{m} .5$ between 1930 and 1940 and slowly by $\sim 0^{m} .5$ until 1983, showing a wavy pattern in the light curve. During the last $30 \mathrm{yr}$, the colour-index $(B-V)_{\mathrm{J}, 0} \sim 0$. Thus during $\sim 1900$ the colour became bluer by at least say $\sim 0^{m} \cdot 65$.

At the right of Figure 20 the scale for $M_{V_{\mathrm{J}}}$ is indicated adopting $r=2800 \mathrm{pc}$ and the foreground extinction $A_{V_{\mathrm{J}}}=1^{m} .55$. The present values for the central star $M_{V_{\mathrm{J}}}(s) \sim-9^{m} .5$ and for ( $\overline{\mathrm{co}}) M_{V_{\mathrm{J}}} \sim-6^{m} .8$ (Section 7) are marked as well as the present bolometric magnitude $M_{\text {bol }}(s) \sim-12^{m} .3$ (Section 8 ). The latter is indicated with a brace, since we assume that this value was also valid during the previous phases, with the exception of the period $\sim 1830$ to 1860 (see further). It is remarkable that the 1843 maximum presumably reached a value of $M_{V_{\mathrm{J}}} \sim-14^{m} .8$, which is in excess by $\sim 2^{m} .5$ compared with the present $M_{\mathrm{bol}}=-12^{m} \cdot 3$. This inconsistency is too large to be caused by errors in the visual estimates, or by an error in the present $M_{\text {bol }}$. In view of the estimated intrinsic colour in the 1843 maximum $(B-V)_{\mathrm{J}, 0} \sim 0^{m} .9$ and if we can treat the envelope as a normal stellar atmosphere, the bolometric correction $B C \sim 0$ and consequently $M_{\text {bol }}(1843) \sim-14^{m} .8$. It cannot be excluded that $\eta$ Car was already an infrared source, unlike that of a normal star, thus $M_{\text {bol }} \leqslant-14^{m} .8$. We will discuss this hypothetical luminosity excess in Section 9.3. However, since a gaseous envelope is not completely comparable with a normal stellar atmosphere we cannot therefore exclude the possibility that the $B C$ was large positive. In the extremest case $+2^{m} \cdot 06$, thus if all flux was radiated in the visual only. This number needs some explanation. We compare the outside atmospheric flux of a star with $m_{\mathrm{bol}}=0$ with that of a star with $m_{\mathrm{vis}}=0$. According to Allen (1973) both fluxes amount to $2.48 \times 10^{-5} \mathrm{erg} \mathrm{cm}^{-2} \mathrm{~s}^{-1}$ and $2.54 \times 10^{-6}$ lux or 
$3.73 \times 10^{-6} \mathrm{erg} \mathrm{cm}^{-2} \mathrm{~s}^{-1}$, respectively. Consequently the flux of a star with $m_{v}=0$ is smaller by a factor 6.67 , than the flux of a star with $m_{\text {bol }}=0$. Thus if during the 1843 outburst all the flux of $\eta$ Car was situated in the $V$ band, one would underestimate the total flux by a factor 6.67 by using a $B C=0$. This corresponds with $2^{m} \cdot 06$, the maximum positive value for $B C$.

At this moment more than $90 \%$ of the radiation comes from the infrared (Section 8 ), because of the redistribution of the ultraviolet flux by the dust, while the visual flux is extremely low. Consequently it is possible that at a certain moment, say between 1820 (star still in a normal S Dor phase) and 1843 (top of visual maximum), the peak of the spectral energy distribution started to shift to the longer wavelengths because of the density increase in the envelope. During this process it should have passed the visual region. Apart from the fact whether this is physically possible, the rather homogeneous structure of the homunculus suggests that it is possibly formed in a relative short time during a period of excessive mass loss around 1840. This is especially conceivable with a luminosity increase by a factor of 10 and a mass loss rate by the stellar wind proportional to $\left(L / L_{\odot}\right)^{1.6}$ (Abbott et al., 1981). Thus the mass loss rate should have increased by a factor of 40 (Section 9.3)! With a presumable average expansion of $\sim 750 \mathrm{~km} \mathrm{~s}^{-1}$ (Section 9.5) the envelope reached in $\sim 150 \mathrm{yr}$ its present diameter of $15^{\prime \prime}$. Thus the hypothetical luminosity excess is evidently connected with the existence of the homunculus and therefore possibly real.

\subsection{AN INTERPRETATION OF THE PAST BEHAVIOUR OF $\eta$ Car}

On account of above discussed historical facts, we propose the following explanation for its behaviour in the last centuries. Eta Car is a very massive $\left(\sim 150 M_{\odot}\right)$ hot star, which may already be in a state of variability for at least $1000 \mathrm{yr}$ (see further). At the time of Ptolemy (AD 135) the star was not important enough to be mentioned, because if not yet variable, it should have been of normal visual brightness adopting that $T_{\text {eff }} \sim 30000 \mathrm{~K}$ viz. $m_{v} \sim 4^{m} .5$ (see Figure 24). From 1600 to 1843 the star is recorded as highly variable from $m_{v}=4^{m}$ to $-1^{m}$. Because of the fact that Walborn et al. (1978) detected nebulosities which must have been expelled long before 1600 , we suppose that the variability already existed before that date. The state of instability apparently reached a climax in the middle of the 19th century. It must be noted that the climax in 1843 was preceded by at least three sharp brightness increases by $\sim 1^{m}$ in the visual with timescales of $\sim 1 \mathrm{yr}$ and intervals of 5 to $10 \mathrm{yr}$.

Since the colour was very red around 1843: $(B-V)_{\mathrm{J}, 0} \sim 0.9$ and in the following deep minimum in 1870 probably bluer: $(B-V)_{\mathrm{J}, 0} \sim 0^{m} \cdot 65$, we infer from this, that this maximum and all previous ones were $S$ Dor type phases with high mass loss rates (see also Section 7). As a result the star is regularly enveloped by a cooler gas shell, which redistributes ultra violet light into longer wavelengths regions such as the visual. Even now we can recognize in the $\mathrm{H} \alpha$ emission line the influence of several expanding massive shells, with velocities of 45,200 , and $600 \mathrm{~km} \mathrm{~s}^{-1}$ (Melnick et al., 1982). This redistribution, which is also observed at the photometrically well studied stars S Dor and R 71 (van Genderen, 1979, 1982) and R 127 (Stahl et al., 1983b), all in the LMC, causes a 
visual light increase of $1^{m}$ to $2^{m}$, but leaving the $M_{\text {bol }}$ more or less unchanged. The cooler gas shell dominates the total appearance of these objects giving rise to a redder colour. At its maximum brightness in $1843 \eta$ Car even reached presumably $M_{\text {bol }} \sim-14.8$, at least $2^{m} .5$ brighter than at present. As a consequence we must assume that the central star was more luminous around 1843 than at present.

Stothers and Chin (1983) discussed a number of possibilities to explain the S Dor mechanism. It is possible that the reason lies in instabilities in the outer stellar envelope. De Jager (1980, his Figure 6) defined a barrier in the HR diagram where supergiants arrive on their evolution away from the main sequence. The turbulent velocities of the outer layers surpass the escape velocity so that matter easily can move away from the star. In this respect we should mention the fast moving nebulosities and condensations, having tangential velocities of $300-1300 \mathrm{~km} \mathrm{~s}^{-1}$ (adopting $r=2800 \mathrm{pc}$ ). They must have left the star mainly in the 15th to 19th century (Gaviola, 1950; Ringuelet, 1958; Walborn et al., 1978). Maeder (1983) and de Jager (1984) presume that such stars cannot surpass this limit, which we shall call the Humphreys-de Jager limit, and always are forced to stay at the left of it, undergoing a number of excessive mass losses, until its core is so far evolved that it moves back to the far blue and at last into the Wolf-Rayet stage. What ever the truth may be, we have to explain the increase of the luminosity of $\sim 2^{m} .5$. If we assume a constant radius, the temperature should have been $\sim 50000 \mathrm{~K}$ to explain the luminosity increase. In Section 9.3 we shall discuss this further and try to estimate the resulting mass loss.

After this phase of unrest has passed, there was a smooth and steady drop in the visual brightness during the next $\sim 30$ yr down to $m_{v} \sim 7 m$. Obviously the strong $\mathrm{S}$ Dor activity stopped at once after the $\sim 30 \mathrm{yr}$ of enhanced surface temperature. It is tempting to suppose that both were connected with each other, although two small outbursts were observed in the deep minimum. (The central star still ejects now and then a new shell, such as presumably in 1981 according to our photometry and the spectroscopy of Zanella et al. (1984), see Section 9.6.) The colour became presumably bluer, since the domination of this envelope decreased with respect to the hot star. So far it was generally assumed that the decline largely resulted from the formation of a dust shell (Andriesse et al., 1978; Viotti and Andriesse, 1981). However, we believe that this was not true, otherwise the colour would have reddened by $\sim 3^{m}$, if the drop in visual brightness of $\sim 9^{m}$ was only caused by dust extinction. The apparent visual brightness in the minimum was $\sim 7^{m} .8$, which is $\sim 0^{m} .8$ fainter than the present value for $(\overline{\mathrm{co}})$, namely $V_{\mathrm{J}}(\overline{\mathrm{co}})=7^{m} \cdot 0$ (Table VI, Figure 20). Besides, $M_{V_{J}}$ of the minimum is $\sim 3^{m} .5$ fainter than that of the central star $\left(M_{V_{J}}(s)=-9^{m} .5\right)$ and the intrinsic colour for such a hot star should be $(B-V)_{J_{0}} \sim-0^{m} .30$. Thus, apparently dust has already been formed in the envelope during the steep decline, perhaps with a higher density in front of the star than at present, in view of the 0.8 difference just mentioned. At a distance of $\sim 100 \mathrm{AU}$ (this is $\sim 1 \mathrm{yr}$ after the maximum), the temperature of the stellar radiation is already lower than $1500 \mathrm{~K}$, the limit below which dust can condensate, depending on its chemical composition.

We therefore believe that the $9^{m}$ drop was a combination of at least three processes: 
$\sim 2^{m} .5$ by the decrease of the luminosity excess, $\sim 3^{m}$ by the decrease of the $\mathrm{S}$ Dor effect, ranging from $M_{V_{J}}=-12^{m} .3$ in maximum down to $M_{V_{J}}=-9^{m} .5$ in minimum and $\sim 3^{m} \cdot 5$ by dust extinction in the envelope. We thus adopt that the star did not change much intrinsically, with the exception of the interval 1830 to 1860 . The reddening by the newly formed dust should have been $\sim 1^{m}$ and with an intrinsic colour of the star of $\sim-0^{m} .30$, the colour in the minimum should have been $(B-V)_{\mathrm{J}, 0} \sim 0^{m} .65$ (the subscript ' 0 ' indicates that a correction has been made for the interstellar foreground reddening).

The F5-type supergiant absorption spectrum in this deep minimum, in the interval 1889-1895, mimiced by the envelope, showed strong $\mathrm{P}$ Cygni profiles, with velocity differences between absorption and emission lines of $\sim 200 \mathrm{~km} \mathrm{~s}^{-1}$ (Whitney, 1952). It indicates that the B-type star was shining through this envelope. Around 1900 the number of emission lines was increasing and the continuing strength and domination of the spectrum (Cannon, 1916) up to now (Aller, 1970) may be indicative of the forming of an important $\mathrm{H}$ II envelope close around the star by a strong stellar wind. The condensations certainly contribute to this emission spectrum.

From 1930 to 1940 the photographic magnitude quickly rose by $\sim 1^{m} .5$. In the visual the rise should have been smaller. In 1952 the first determination of the colour by de Vaucouleurs and Eggen (1952) resulted in $(B-V)_{\mathrm{J}_{0}} \sim 0$ (see caption Figure 20), thus the object was in 1940 presumably bluer than in 1930 by the order of say $\sim 0^{m} \cdot 6$. Thus the rise in visual was presumably $<0^{m} .9$ (see further).

For hot stars $(B-V)_{\mathrm{J}}$ is very insensitive to temperature variations. Consequently the bluing must be largely attributed to a decrease of the dust density in front of the star. If this was dust close to the star, say in a circumstellar ring (Warren-Smith et al., 1979; their model is shown in our Figure 15), such a decrease may have had consequences, not only for the light received from the central object, but also for that of the homunculus.

In this connection, it is of interest to mention the following two facts. Firstly, the homunculus was discovered as late as 1933 by van den Bos (1938) and described by him as 'red'. Secondly, Thackeray (1953) noticed that in 1951 the visual line intensities of the nucleus, have not been changed to any marked degree compared with the Cape and Lick spectra of some $40 \mathrm{yr}$ before. Therefore he supposed that the brightness increase between 1930 and 1940 was caused by the brightening of the homunculus.

Thus we conclude that between these years the dust density close around the star decreased, giving rise to less extinction of (co). The visual brightness rose presumably by $\leqslant 0 m .9$. The difference of $0^{m} .8$ between $(\overline{\mathrm{co}})$ at present and in say 1900 (assuming that the reflecting power of the homunculus was not well developed) would be in accordance with this value, were it not that since 1950 the visual brightness has risen by $\sim 0^{m} .5$. Thus the true visual rise between 1930 and 1940 would than be only $\sim 0^{m} 3$, if all variations were only caused by the star. The bluing by $\sim 0^{m} \cdot 6$ is too large with respect to this estimate and cannot thus be explained by a decrease of extinction in front of the star alone. Apart from the fact that the uncertainties in all these numbers are a few tenths of a magnitude, we presume that the homunculus brightened too (as Thackeray supposed), with a bluing of the scattered light.

In 1946 a small maximum occured, perhaps caused by another small outburst in view 
of the presence of a condensation close to the central star (Ringuelet, 1958). Hereafter the visual brightness rose slowly with a wavy pattern, showing a characteristic time of 2-3 yr (Section 3). The colour is despite the further increase in visual light by $\sim 0^{m} .5$ still more or less the same. During the last $10 \mathrm{yr}$ the object as a whole became slightly more ultraviolet, viz. $\sim 0^{m}$. 1 (Section 3). A precise interpretation of this phenomenon is so far impossible, since there are a combination of several changing conditions. We should mention here the varying scattering and extinction characteristics of the dust in the homunculus and the growing $\mathrm{H}$ II shell close around the star.

The small amplitude variations (the wavy pattern in the visual light curve) will be discussed in Section 9.6.

\subsection{A POSSIBLE EXPLANATION FOR THE EXCESS LUMINOSITY AROUND 1840 AND AN ESTIMATION OF THE MASS LOSS}

The observation discussed above (Sections 9.1 and 9.2) show that the central star presumably brightened between 1830 and 1860 by $\sim 2.5$ bolometric magnitudes and reached a maximum of $M_{\text {bol }} \sim-14^{m} .8$ in 1843. An increase of the surface temperature by $\sim 20000 \mathrm{~K}$ up to $\sim 50000 \mathrm{~K}$ could explain the excess luminosity (Section 9.2 ). This implies an increase in luminosity during the maximum visual brightness to $L \sim 7.8 \times 10^{7} L_{\odot}$. The present luminosity is $6.6 \times 10^{6} L_{\odot}$ (Section 8$)$. It is clear that the amount of energy released during the outburst is so large (of the order of $2.5 \times 10^{50} \mathrm{erg}$ ) that the origin of it must be related to the stellar interior and not to an atmospheric instability. We do not know the mechanism for the instability in the star which produces such a large amount of energy, but we can estimate its effect on the atmosphere of the star.

The mass loss rate of luminous early type stars depends mainly on the stellar luminosity. Abbott et al. (1981) found that $\dot{M} \sim 4 \times 10^{-15}\left(L / L_{\odot}\right)^{1.6} M_{\odot} \mathrm{yr}^{-1}$ for early type supergiants. Other relations have been proposed by e.g. Lamers (1981) in which the gravity is also taken into account. However for the present purpose we will assume that Abbott et al.'s relation for supergiants is applicable to the very luminous central star of $\eta$ Car. During the maximum brightness of 1843, the luminosity was a factor 10 higher than the present one. This implies that the mass loss rate during that time was about 40 times larger than at present. Assuming a present mass loss rate of $\sim 10^{-4} M_{\odot} \mathrm{yr}^{-1}$, the rate could have been as large as $4 \times 10^{-3} M_{\odot} \mathrm{yr}^{-1}$ during maximum brightness. We shall return to these estimations further in this section.

It is clear that such a high mass loss rate would change the characteristics of the wind drastically. For instance, one can expect that the star will be unable to keep such a dense wind ionized, so that recombination and cooling of the wind will occur. Such an effect is observed in the wind of P Cyg (Lamers et al., 1984). When P Cyg ejects a high density shell, the degree of ionization in the shell is smaller than in the normal wind and the temperature of the shell is also lower than in this wind. Such an effect is expected to have occurred also during the active phases of $\eta$ Car. (The F-type spectrum observed around 1890, see Section 9.1, is possibly due to the same effect.) If the wind has recombined and was as cold as about $10000 \mathrm{~K}$, it would be optically thick in the Balmer 
and Paschen continuum. This would explain the 'reddish' colour of the object during maximum brightness.

This interpretation can qualitatively explain the observations during the phase of maximum brightness by means of a strong increase in luminosity. It leaves open the origin of this luminosity increase. Stothers and Chin (1983) have investigated various possible mechanisms for the variations in S Dor variables. They conclude that two of these mechanisms can most likely explain the observations: firstly, a sudden increase in the surface mass loss, and, secondly, envelope energized pulsations of a star which is close to the Eddington limit $\left(L_{\mathrm{E}}\right)$. We think that the first possibility may indeed be able to explain the observations, but that it does not answer the question of the reason for the enhanced mass loss. The arguments given above show that the brightening of the star requires too much energy to be caused by atmospheric instabilities. Therefore the second possibility of the envelope-energized pulsations is more likely. The dynamical timescale of the pulsations is of the order of

$$
\tau_{\text {dyn }}=\left[\left(\frac{L}{L_{\mathrm{E}}}-1\right) \frac{G M}{R^{3}}\right]^{-1 / 2} \mathrm{day}
$$

(e.g. Sparks et al., 1978).

Assuming a mass of the order of $\sim 150 M_{\odot}$ and a radius of $\sim 100 R_{\odot}$, we find

$$
\tau_{\text {dyn }} \sim 1.52\left(\frac{L}{L_{\mathrm{E}}}-1\right)^{-1 / 2} \text { day. }
$$

If we take the observed timescale of the order of a year, we find that $L / L_{E} \sim 1$. Thus $L$ is very close to the Eddington limit and as soon as $L>L_{\mathrm{E}}$ the excess radiation will drive off matter on the dynamical timescale.

Stothers and Chin have shown that such instabilities are expected to occur for stars with $L>10^{6} L_{\odot}$, which is very close to the observed limit of the S Dor variables. Moreover if the star is close to the Eddington limit, the ejection of mass is likely to occur in random outbursts, as argued by Stothers and Chin.

Thus it is possible that the luminosity excess was also due to envelope-energized pulsations when the star's luminosity was close to its Eddington limit. After the major maximum in 1843 the star's luminosity dropped apparently far below this limit and came at rest (temporarily, since in 1870 and 1890 two outbursts with amplitudes of $\sim 1^{m}$ were recorded). During the following $\sim 30 \mathrm{yr}$ the visual light curve dropped very smoothly by $\sim 9^{m}$ (Figure 20, see also the compilation of visual brightness estimations of Innes (1903)). Bolometrically the drop was presumably only $\sim 2^{m} .5$ down to $M_{\text {bol }} \sim-12^{m} \cdot 3$, its present value. Apparently the spectral energy distribution changed drastically during this time, in favor of the ultraviolet and infrared parts of the spectrum.

We assume that the central star is now more or less normal and that it looses mass at a lower rate by a normal stellar wind. An upper limit can be estimated with the aid of the condition that the photon momentum flux is equal to the mass momentum flux (de Jager, 1981):

$$
\dot{M} v_{\infty} \leqslant \frac{L}{c} .
$$


With $v_{\infty} \sim v_{\text {esc }}$ for stars like $\eta$ Car (de Jager, 1984) and using $v_{\text {esc }} \sim 750 \mathrm{~km} \mathrm{~s}^{-1}$ (Section 9.5), the mass loss rate at present amounts to:

$$
\dot{M} \sim 1.8 \times 10^{-4} M_{\odot} \mathrm{yr}^{-1} .
$$

We have also used the Lamers (1981) empirical formula (his Equation (21)), in which $\dot{M}$ is a function of $M, L$, and $R$ :

$$
\dot{M} \sim 10^{-4} M_{\odot} \mathrm{yr}^{-1}
$$

which is of the same order. (However, it will decrease by a factor of five if the minimum values for $M, L$, and $R$ are used in the case that the system consists of three stars of equal luminosity.) As explained before, we expect that the mass loss rate at present is presumably a factor of 40 smaller than during the major outburst around 1840. Since we presume that the homunculus is mainly built up of material expelled $150 \mathrm{yr}$ ago (Section 9.1), within the interval $\sim 1830$ to 1860 , then in the $\sim 30 \mathrm{yr}$ interval the total mass should be $\sim 0.12 M_{\odot}$. Together with the mass expelled during the last century and keeping in mind that still shells are ejected now and then (Melnick et al., 1982; Zanella et al., 1984; Section 9.6) the total mass is estimated to amount to:

$$
M_{\text {hom }} \sim 0.15 M_{\odot}
$$

One could argue that this still may be an underestimate, since the star is at least since 1600 onwards an S Dor variable. During such a phase, well observed stars like R 71 and $\mathrm{R} 127$ loose mass by a factor of 5 more than during the quiscent stages. This number is obtained by comparing the observed mass losses in the maximum state found by Wolf et al. (1981) and Stahl et al. (1983b), respectively, and those for the minimum state computed with the aid of Equation (21) of Lamers (1981). If this also applies to $\eta$ Car, the mass loss rate during the $\mathrm{S}$ Dor phases before 1820 amounts to:

$$
\dot{M} \sim 5 \times 10^{-4} M_{\odot} \mathrm{yr}^{-1} .
$$

As said before we do not think that much of this material is part of the homunculus because of dynamical and morphological reasons. None of the other still active S Dor type stars in LMC, M31, and M33 (in total \pm 15 specimen) show any sign of a dust envelope of the type of the homunculus in view of the non-exceptional infrared radiation (Glass, 1974, 1984; Allen and Glass, 1976; Humphreys et al., 1984). Thus $\eta$ Car is unique not only in view of the bi-polar type reflection nebula with a relative high density and homogeneously shaped, but also in view of its excess luminosity and subsequently drop into a relative quiet state.

Our value of $\dot{M}$ for the major outburst around 1840 is lower by a factor of 20 and 5 compared to those of Andriesse et al. (1978) and Hyland et al. (1979) viz. 0.08 and $0.02 M_{\odot} \mathrm{yr}^{-1}$, respectively. In the first reference the authors interpreted the steep decline, using spectral data on the gas and infrared data on the dust, in terms of a condensation rate into dust. However, we consider the visual light curve as an effect of three different processes (Section 9.2). Thus the steep decline after 1843 is in our opinion not an effect of dust condensation alone. The value of $\dot{M}$ of Hyland et al. is 
based on the dust model of Mitchell and Robinson (1978) with $M_{d}=0.03$ (see also Section 7) and a gas to dust ratio of 100. The total masses for the homunculus derived by Andriesse $e t$ al. and Hyland et al. are consequently much higher than our's: 10 and $3 M_{\odot}$, respectively.

Stothers and Chin (1983) even employed $\dot{M}=0.3 M_{\odot} \mathrm{yr}^{-1}$ in their merely illustratively ment models for explaining the $\mathrm{S}$ Dor phenomenon in a star of $60 M_{\odot}$.

An important step ahead would be made concerning the mass loss problem, if the total mass of the homunculus could be derived independently.

\subsection{A COMPARISON WITH P Cyg}

It is of interest to summarize the evolutionary scenario of the less luminous star P Cyg $\left(M_{\text {bol }}=-9^{m} .9\right)$ proposed by Lamers et al. (1983) and to investigate its photometric history. Lamers et al. proposed a scenario in which P Cyg is now for the second time a blue supergiant after having been a red supergiant a few $10^{4} \mathrm{yr}$ ago. In that phase one or more shells of matter have obscured the star, of which the remnants are now observed as an arc-shaped radio structure at a distance of $0.65 \mathrm{pc}$ from the star (Wendker, 1982). During its evolution to its present position in the HR diagram the star still ejected matter in outbursts, until it emerged through this shell around 1600. The irregular brightness should then be caused by inhomogenities in the dust shell. On the other hand, Wendker (1982), thinks that this is the material ejected in the outbursts of 1600 to 1650 . This would imply a mean expansion velocity of $1200 \mathrm{~km} \mathrm{~s}^{-1}$, which according to Lamers et al. is too high by a factor of four compared to the present stellar wind velocity of P Cyg. Therefore they are inclined to believe that the first explanation is the most likely. However, we think that P Cyg was already active long before the first data of discovery (1600 AD) and that it underwent a number of S Dor type outbursts of which the expel-velocities were consequently $<1200 \mathrm{~km} \mathrm{~s}^{-1}$. Condensations expelled by $\eta$ Car have tangential velocities of $300-1300 \mathrm{~km} \mathrm{~s}^{-1}$, which are of a comparable size. Nevertheless it is also quite possible that the arc-shaped structure is the remnant of the red supergiant phase and that the gas expelled around 1600 and before that date is of such a small mass, that it is undetectable with present astronomical techniques.

Figure 21 shows the photometric history of P Cyg schematically according to Zinner (1952), see also de Groot (1969). The star was 'red' during the maxima of the 17th century, while at present $(B-V)_{\mathrm{J}, 0} \sim 0^{m} .2$ adopting $E(B-V)_{\mathrm{J}}=0^{m} .63$ (Lamers et al., 1983) making a yellow-orange impression. With $r=1800 \mathrm{pc}$ the maxima have $M_{V_{\mathrm{J}}} \sim-10^{m} .2$ and if we suppose that $B C \sim 0$, because of a continuum presumably of the same type as $\eta \mathrm{Car}, M_{\mathrm{bol}} \sim-10^{m}$. , which is close to its present luminosity of $-9^{m} .9$ (Lamers et al.). Thus although the assumption of the $B C$ is rather crude, we find support for our view that P Cyg underwent S Dor type outbursts similar to S Dor, R 71, R 127, and $\eta \mathrm{Car}$. We also note that no important excess luminosity was present at the observed brightness maxima of P Cyg. It is therefore not surprising that no 'homunculus-type' envelope is present.

Similar to $\eta$ Car, P Cyg slowly increased in brightness after the deep minimum and showed irregular visual brightness variations of $\pm 0^{m} .2$ (de Groot, 1969). Nightly and 


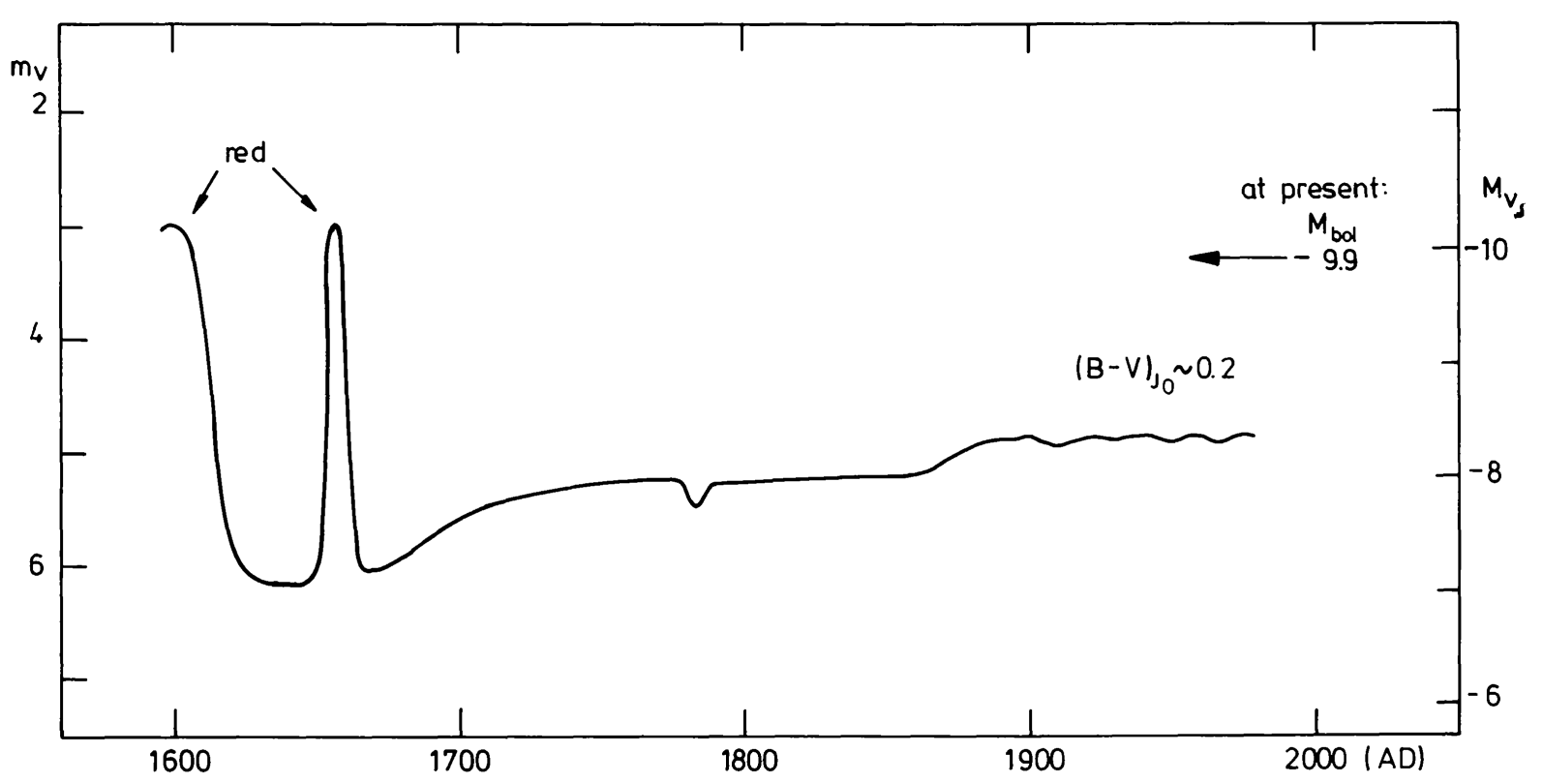

Fig. 21. A schematic representation of the photometric history of P Cyg.

monthly monitoring in 1943 by Groeneveld (1944) revealed light variations of various time scales with amplitudes in the order of $0^{m} \cdot 05$ to $0^{m} \cdot 10$ (see Note added in proof).

Lamers et al. thus presume that P Cyg is in a late post-Main Sequence phase. This seems to be supported by the observed abundances of $\mathrm{N}$. The same can probably be said of $\eta$ Car. The observed $\mathrm{O} / \mathrm{N}$ and $\mathrm{C} / \mathrm{N}$ ratios by Davidson et al. (1982) support the post-Main Sequence state of $\eta$ Car. However, according to Melnick et al. (1982), the spectral differences may also be caused by different exitation mechanisms and not by abundance effects. On the contrary, new evidence has been put forward by Davidson et al. (1984), that $\eta$ Car is evolved and that mixing has occurred: the helium abundance by mass of the ejecta and thus of the outer layers of the central star, amounts to $Y=0.42$.

\subsection{SOME CHARACTERISTICS OF S DOR TYPE STARS AND THEIR POSITION IN THE THEORETICAL HR DIAGRAM}

Figure 22 shows the theoretical HR diagram with a few evolutionary tracks according to Maeder (for mass loss case B). Small numbers between brackets refer to the references mentioned in the caption. The three well observed S Dor type stars: S Dor, R 71, and R 127 are shown during their minimum brightness, thus when no gas envelope of any importance is present. The position of S Dor is not quite certain, but is based on an extrapolation. The luminosities of $\mathrm{P}$ Cyg and $\zeta^{1}$ Sco are based on newly revised values and both have no important envelopes as well. The latter is not known as an S Dor type star, but in view of its small scale variability with probably two kinds of mass loss processes, made it interesting to show its position also (Sterken, 1977; Burki et al., 1982). It should be noted that the minimum luminosities of S Dor and R 71 are $\sim 1^{m}$ and $\sim 0^{m} .5$ lower, respectively, than those in the maximum (see references). However if a larger value for the reddening is applied, the minimum luminosity equals more the 


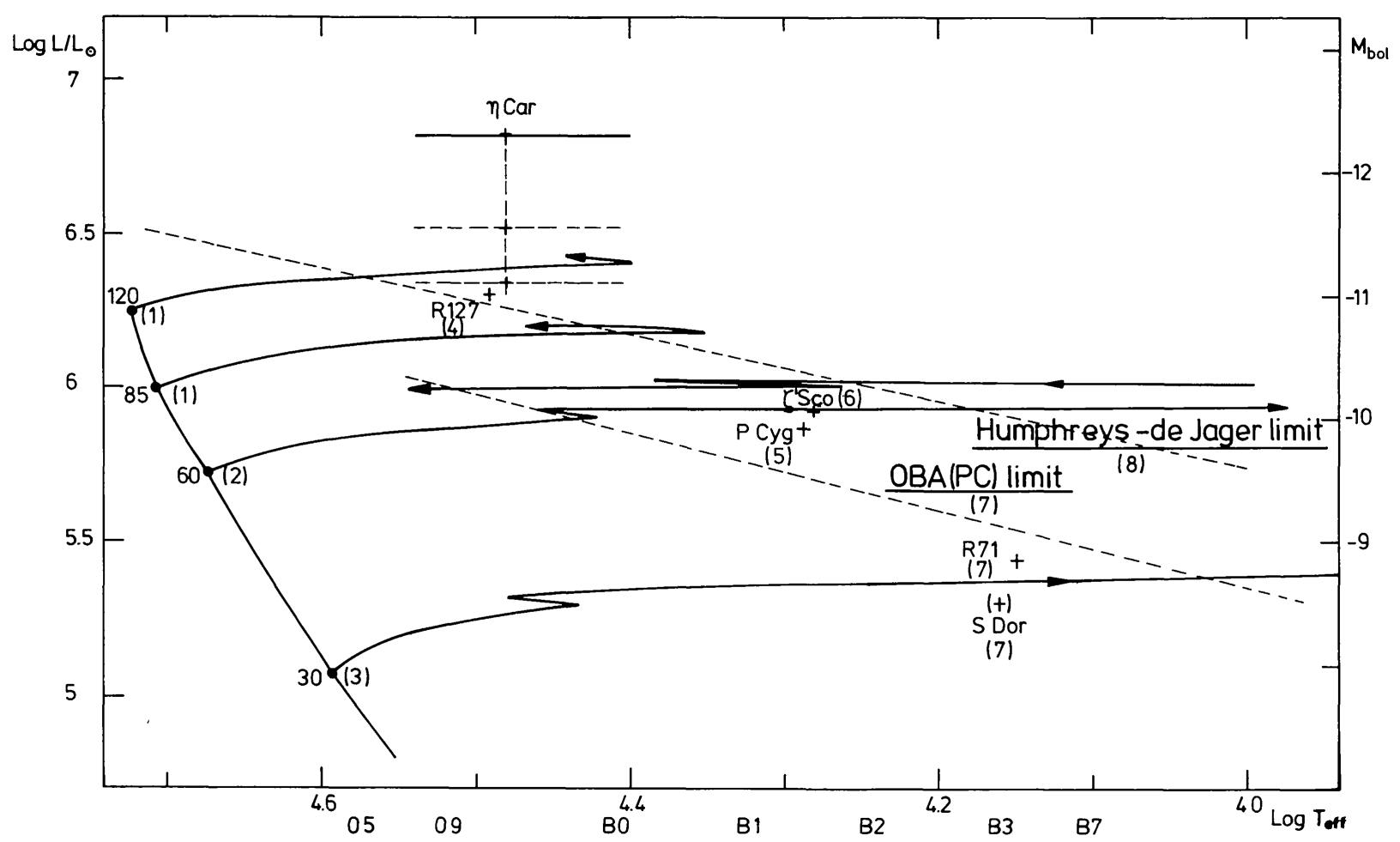

Fig. 22. The theoretical HR diagram with four well studied S Dor type stars (P Cyg, R 127, R 71, and S Dor in their minimum state) and the variable hypergiant $\zeta^{1}$ Sco. Bracketed numbers refer to the following references: (1) Maeder (1980); (2) Maeder (1981a); (3) Maeder (1981b); (4) Stahl et al. (1983); (5) Lamers et al. (1983b); (6) $\log L / L_{\odot}$ from van Genderen et al. (1984), $\log T_{\text {eff }}$ from Humphreys (1978); (7) van Genderen (1982) and van Genderen et al. (1983); (8) De Jager (1984).

maximum luminosity (van Genderen, 1979). The position of $\eta$ Car is shown by adopting for the central star $T_{\text {eff }}(s)=30000 \pm 5000 \mathrm{~K}$ and $\log L / L_{\odot}(s)=6.82$ (also the minimum luminosities are indicated in case $\eta$ Car is a double or triple star). Two limits are shown (dotted lines), the Humphreys-de Jager limit of stellar existence and the OBA(PC) limit above which supergiants start to show PCyg profiles $\left(\dot{M}>2 \times 10^{-6} M_{\odot} \mathrm{yr}^{-1}\right)$ in the optical region. Both limits run nearly parallel.

Two questions arise. The first one is whether the $S$ Dor phenomenon only occurs for a few massive stars because of special circumstances, or whether all massive stars pass this stage once or more times, but for a very short time. Stahl et al. (1983a) for example found evidence that massive stars may spend about $20 \%$ of the B-supergiant phase as drastic mass loosers, after which they end into the Wolf-Rayet stage. The second question already broached in Section 9.2 is: what is the cause of the outburst? Is the $\mathrm{S}$ Dor type outburst caused by sudden instabilities in the outer layers as proposed by Stothers and Chin (1983), or by shock driven mass loss, which according to de Jager (1984) occurs in hypergiant atmospheres, or are S Dor type stars common envelope binaries of which it is estimated that only a few tens exist in the galaxy (cf. Tutukov, 1981)? An interesting phenomenon of S Dor type stars is their low expansion velocity compared to the stellar wind velocities for normal $O$ and B type stars: 3000 to $1500 \mathrm{~km} \mathrm{~s}^{-1}$. For S Dor, R 71, and R 127 the expansion velocities during the active 
phases are at least a factor of 10 lower (Thackeray, 1974; Wolf et al., 1980; Stahl et al., 1983b).

Recently Lamers et al. (1984) found evidence that stellar winds can be caused only by radiation pressure. The consequence is that additional mass loss caused by the $\mathrm{S}$ Dor phenomenon will increase the density of the stellar wind and slow down the expansion velocity by conservation of momentum, since the photon flux stays the same. One would then expect that the mass loss of the stars mentioned above should be increased by at least a factor of 10 compared to the quiescent phase. For R 71 and R 127, only a factor of 5 was found (Section 9.3). The expansion velocity is thus two times lower than one would expect.

At preent P Cygni's mass loss rate is $\dot{M} \sim 2.5 \times 10^{-5} M_{\odot} \mathrm{yr}^{-1}$ (Van Blerkom, 1978; Abbott et al., 1980; White and Becker, 1983), while according to Equation (21) of Lamers (1981) it should be $\sim 2.5$ times smaller. The present wind velocity is indeed lower by roughly the same amount (Lamers et al., 1983).

For $\eta$ Car the problem of the expansion rate is more intricate. The different parts of the homunculus (such as the condensations and the different shells) show different expansion velocities, from $\sim 40$ to $1300 \mathrm{~km} \mathrm{~s}^{-1}$ (e.g. Thackeray, 1961; Craine, 1974; Walborn et al., 1978; Melnick et al., 1982; Zanella et al., 1984). Expansion velocities up to $\sim 700 \mathrm{~km} \mathrm{~s}^{-1}$ in the small nebula close around the central star has been detected by Cassatella et al. (1979) with the aid of a high resolution ultraviolet spectrum made with IUE in 1978. We shall take $750 \mathrm{~km} \mathrm{~s}^{-1}$ as a reasonable average (then the present radius of $\sim 8^{\prime \prime}$ or $0.1 \mathrm{pc}$ has been reached in $\sim 150 \mathrm{yr}$; it is also roughly the escape velocity). This value is about a factor of 4 lower than the expected wind velocity for a star with $T_{\text {eff }} \sim 50000 \mathrm{~K}$, viz. $\sim 3000 \mathrm{~km} \mathrm{~s}^{-1}$ (according to de Jager's (1981) empirical diagram). Yet the mass loss rate for the major outburst around 1840 was estimated to be 40 times higher than the normal state (Section 9.3). Thus in view of the ratio's between the mass loss rates in active and non-active state, the expansion velocities of $\mathrm{R} 71$ and $\mathrm{R} 127$ are a factor of 2 too low, that of P Cyg (at present in a relative quiescent phase) is roughly correct and that of $\eta$ Car a factor of 10 too high. It must however be emphasized that all figures on the mass loss rates are uncertain with at least a factor of two.

The large deviation for the situation of $\eta$ Car may perhaps be a support for the envelope-energized pulsations acting around 1840 and which probably gave rise to an extra momentum to the envelope.

\subsection{AN INTERPRETATION OF THE RECENT BEHAVIOUR OF $\eta$ Car}

The small amplitude variations of $\Delta \mathrm{V}_{j} \sim 0^{m} .2$ with time scales of 2-3 yr (Sections 3 and 9 ), caused a wavy pattern in the light curve of $\eta$ Car during at least $\sim 30 \mathrm{yr}$. Figure 8 shows the details during the interval 1974-1983. In order to get more information on the possible cause, the behaviour of the Balmer jump $(D)$ is of importance. For the study of this behaviour the $V, B, U$, and $W$ intensities were transformed to monochromatic absolute fluxes using the calibrations of Lub et al. (1978). Their fluxes, given in $\mathrm{erg} \mathrm{cm}^{-2}$ $\mathrm{s}^{-1} \AA^{-1}$, were transformed into erg $\mathrm{cm}^{-2} \mathrm{~s}^{-1} \mathrm{~Hz}^{-1}$. Then the logarithm of these fluxes 
$F$ were plotted against $1 / \lambda_{\text {eff }}\left(\mu \mathrm{m}^{-1}\right)$ for the four passbands. Since the size of $D$ is slightly sensitive to the reddening, we employed the foreground reddening $E(B-V)_{\mathrm{J}}=0.50$ equivalent to $E(V-B)=0.195$ (Section 7). It turns out that $D$ decreases by only 0.0024 , if $E(V-B)$ is smaller by 0.01 . The corrected Balmer jump $D_{0}$ is then read off at $1 / \lambda=2.70\left(\mu \mathrm{m}^{-1}\right)$ between the extrapolated lines going through $\log F_{V}$ and $\log F_{B}$ and through $\log F_{U}$ and $\log F_{W} . D_{0}$ is determined for a number of maxima and minima in the light curve of Figure 8. The results are listed in Table $X$, together with the approximate Julian Dates, the successive changes in $V(\Delta V$ in log intensity scale; positive and negative means a rise and a drop in brightness, respectively) and $D_{0}$ with an estimated uncertainty of \pm 0.003 . The fourth column lists the successive changes in $D_{0}\left(\Delta D_{0}\right)$. Generally there is a clear anti-correlation between $\Delta V$ and $\Delta D_{0}$. When the brightness rises, the Balmer-jump decreases, except from midway the rising branch 1980/1981 to the maximum at the end of 1981 (JD 2444950 in Table X).

TABLE $X$

The Balmerjump variations as a function of the light variations

\begin{tabular}{lccccr}
\hline $\begin{array}{l}\text { J.D.- } \\
2440000\end{array}$ & $\Delta V$ & $D_{0}$ & $\Delta D_{0}$ & $-\Delta V / 0.032 \times 0.0024$ & {$[B-U]$} \\
\hline 2525 & 0 & 0.174 & 0 & 0 & 0.053 \\
2890 & +0.082 & 0.146 & -0.028 & -0.006 & 0.026 \\
3225 & -0.042 & 0.151 & +0.005 & +0.003 & 0.034 \\
3350 & -0.050 & 0.178 & +0.027 & +0.004 & 0.053 \\
3660 & +0.032 & 0.136 & -0.042 & -0.002 & 0.022 \\
4675 & +0.096 & 0.126 & -0.010 & -0.007 & 0.008 \\
4950 & +0.032 & 0.144 & +0.018 & -0.002 & 0.021 \\
5270 & -0.040 & 0.136 & -0.008 & +0.003 & 0.015 \\
5490 & +0.020 & 0.116 & -0.020 & -0.001 & -0.008 \\
\hline
\end{tabular}

The anti-correlation is normal for stellar continua: the hotter the star, the smaller $D$ is. The range of $D_{0}$ varies within the range of B8 to B3 stars. This is illustrated in Figure 23, where $D$ for a number of hot supergiant stars are plotted against the extinction independent colour index

$$
[B-U]=(B-U)-0.63(V-B) \text {. }
$$

[ $B-U]$ is listed in the last column of Table $\mathrm{X}$. The variation of $D_{0}$ for the $\mathrm{S}$ Dor type stars S Dor and R 71 during their outbursts (see Figure 15 in van Genderen, 1979) are also plotted.

Thus according to the evidence presented here, we tentatively suggest that the small brightness variations are mainly caused by temperature variations of the central star. However, the trend of $D$, from midway the rising branch to the top of the maximum in 1981 (Figure 8), behaved as if suddenly redistribution of ultraviolet light into the visual occurred, thus a similar process as during the outbursts of $\mathrm{S}$ Dor and $\mathrm{R} 71$. While the $V_{\mathrm{J}}$ curve still rose up to the end of 1981 , the $B-U$ and $U-W$ curves went through 


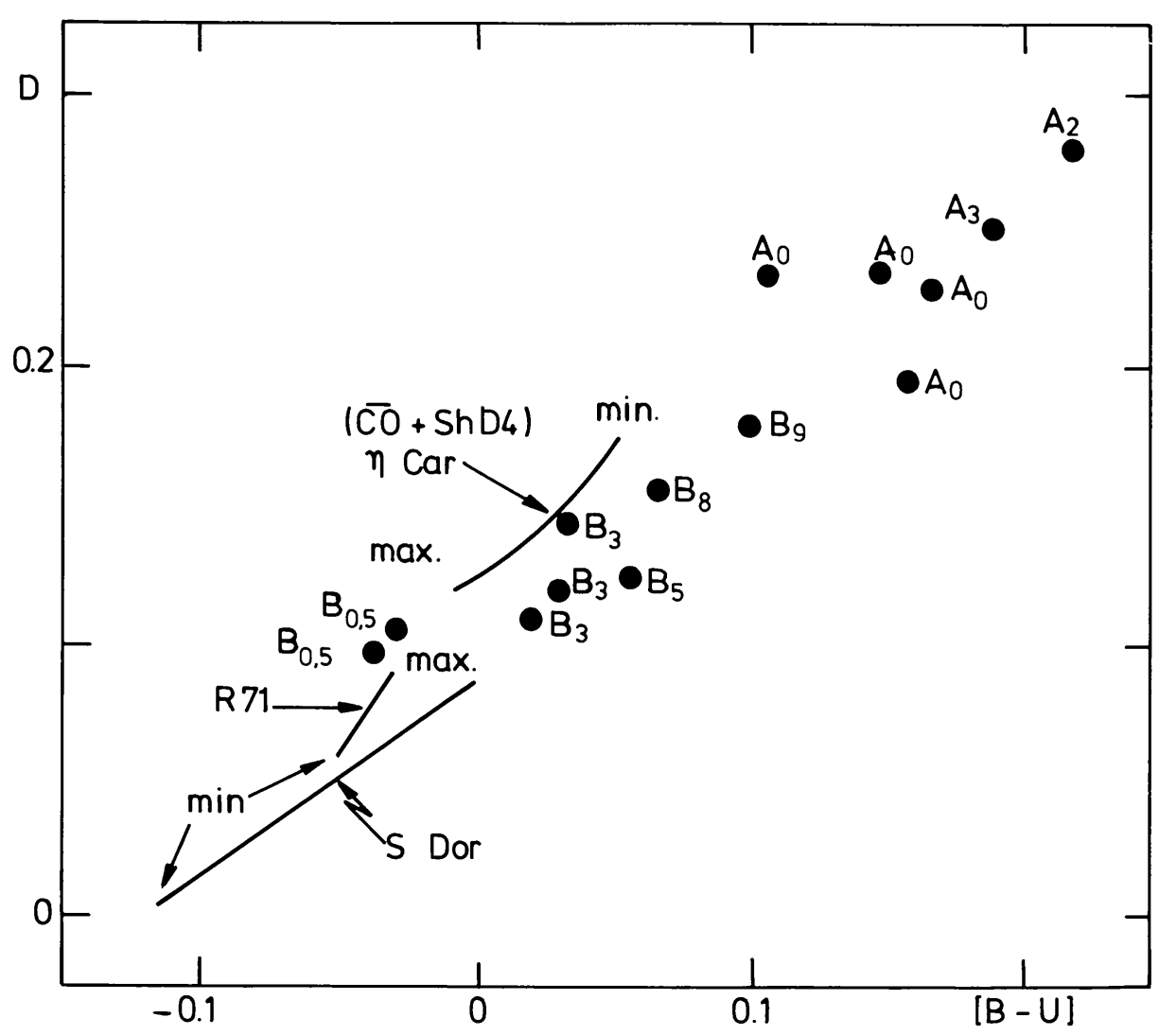

Fig. 23. The relation between the size of the Balmerjump $D$ (in log intensity scale) and the reddening independent colour index $[B-U]$ for a number of hot supergiant stars. The variation of $D$ for $\eta$ Car during the time interval 1974 to 1983 is schematically indicated.

a minimum (thus these colour indices became redder and $D$ increased). Thus it seems that the stellar temperature rose till the middle of 1981, after which it expelled a new shell. This conclusion is in good agreement with the spectroscopic study of Zanella et al. (1984). They noticed in the spectra, made at the end of 1981, a strengthening of P Cygni characteristics and an almost complete disappearance of some high energy lines. They conclude that these effects were caused by an additional expanding shell with a maximum velocity of $\sim 600 \mathrm{~km} \mathrm{~s}^{-1}$, which screened off the ionizing ultraviolet photons of the central star. They believe that this screening also reduced the destruction of dust grains and that therefore the infrared radiation in the $J H K L$ bands, received from the homunculus, increased. We believe however, that this increase, which started at the beginning of 1981 (Figure 9), was caused by the rise of the star's temperature. As soon as the temperature dropped midway 1981, the infrared fluxes dropped also (Figure 9). On the contrary the flux in the $V_{\mathbf{J}}$ band kept on rising because of the redistribution process explained before.

With the aid of the Kurucz (1979) models, it can be deduced that if a star with a gravity $\log g=3.5$ rises from $T_{\text {eff }}=30000$ to $35000 \mathrm{~K}$, the flux in the visual rises by $\sim 0{ }^{m} .25$. This is just the maximum range we observe. Since the radius may also be variable, the real temperature fluctuations will be likely smaller, but presumably still in the range of a few thousand degrees. 
It must be noticed that the expansion velocity of the 1981 shell is also rather high $\left(\sim 600 \mathrm{~km} \mathrm{~s}^{-1}\right)$ compared to the other S Dor type stars. Perhaps some kind of pulsation is still present, giving an extra momentum to the shell.

Hyland et al. (1979) suggest that the small light variations may be due to rotational motion of a non uniform ring or disk of dusty material. Although the variations of $D$ suggest another cause, we will compute these as if they were caused by a variable extinction and compare them with the observed ones. It was mentioned above that $D_{0}$ decreases by 0.0024 , when $E(V-B)$ is lower by 0.01 , or when $A_{V}$ becomes 0.032 smaller. Thus if we interpret a brightness increase with a decrease in $A_{V}$, the corresponding decrease in $D_{0}$ is $\Delta D_{0}=-\Delta V / 0.032 \times 0.0024$. The computed variations $\Delta D_{0}$ are listed in the fifth column of Table X. It is evident that the observed variations are often much larger than the computed ones. Consequently it is not likely that the light variations are caused by a variable dust extinction.

Whitelock et al. (1983) suggest that only a variable extinction by larger grains $(\sim 2 \mu \mathrm{m})$ could cause the infrared variability. Since the grains are probably small (Warren-Smith et al., 1979, see also Section 7 of this paper), their alternative suggestion of a varying temperature of the central star is more likely. The fact that the $H-K$ and $K-L$ colours became steadily bluer with time as $\eta$ Car brightened, fits within this picture: the hotter the star the more the dust temperature rises. The comparable amount of variations of visual and infrared light, even supports that more than one component in the system is varying and that these variations are related (Whitelock et al.). It is conceivable that the $\mathrm{H}$ II shell surrounding the star changes its emission as the temperature of the exciting star changes.

That the problem is indeed complicated because of the degrading of the energy into the various components of $\eta \mathrm{Car}$, is illustrated by the fact that the variation of the colour index $V-B$, although very small: $\sim 0^{m} \cdot 03$, is anticorrelated with $V$. Thus if $V$ brightens, $V-B$ becomes redder. However, at the high temperature of the central star $V-B$ varies very little with $T_{\text {eff }}$ anyway. In this respect, relative emission line strength variations can easily change the behaviour of $V-B$ into a small opposite behaviour. The same holds for the variations in the other colour indices: the variations in $B-L$ and $B-U$ are often slightly larger than one would expect, while $U-W$ shows an anticorrelation with $V$.

Therefore, our main conclusion is that the small amplitude variations with time scale of 2 to $3 \mathrm{yr}$ and possibly also the secular increase of $V_{j}$ since 1952, might be caued by temperature variations and a net rise in temperature of the central star, respectively. These temperature variations may be in the order of a few thousand degrees.

\section{Conclusions}

Eta Car is one of those unique astronomical objects, which have attracted many astronomers to study its characteristics and peculiar behaviour (Section 1). A decade of photometric monitoring of $\eta$ Car in the $V B L U W$ system shows smooth light- and colour curves with several maxima having scales of 2 to $3 \mathrm{yr}$. Light- and colour amplitudes amount to at most $0^{m} \cdot 2$ and $0^{m} \cdot 15$, respectively (Sections 2 and 3 ). 
Scanning of the surrounding reflection nebula (the 'homunculus') and multi-aperture observations revealed the photometric parameters of the homunculus and the central object separately. The latter is a hot massive star $\left(\sim 150 M_{\odot}\right)$ embedded in a cooler gas envelope. At the east-side of the north-south direction the homunculus is bluer in all colour indices than at the west-side. This suggests that the homunculus is built up not uniformly (Section 4.1). Its integrated brightness and average colour is brighter and bluer than that of $(\overline{\mathrm{co}})$, the central object attenuated by the intervening material of the homunculus (Section 4.2).

The foreground reddening, estimated with the aid of the two-colour diagrams, amounts to $E(B-V)_{\mathrm{J}}=0^{m} .50$ (Sections 5 and 7). The reddening suffered by the light of (co) due to dust in the homunculus is estimated to be $E(B-V)_{\mathrm{J}}<0^{m}$. 6 , and perhaps even $<0^{m}$.3. However this value is perhaps not representative for the whole homunculus because of a possible non-uniform density distribution of the material (Section 7).

A comparison with the photometric characteristics of the S Dor type stars S Dor and R 71 (= HDE 269006), suggests that the central star of the object $\eta$ Car may also have undergone a large number of S Dor type outbursts before 1800 (Figure 24). The expanding cooler dense gas shell presumably redistributed ultraviolet light into the visual entailing the enormous rises in visual brightness in the 18th and 19th century (Sections $7,9.2,9.3$, and 9.5). This appreciable change of the spectral energy distribution by a dense cooler gas envelope is called an ' $\mathrm{S}$ Dor effect'.

The integration of the spectral energy distribution from $0.15<\lambda<175 \mu \mathrm{m}$ based on observations made by various authors, reveals a total observed flux corrected for foreground extinction $\left(E(B-V)_{\mathrm{J}}=0^{m} .50\right)$ of $F^{\prime}=(26.5 \pm 0.2) \times 10^{-9} \mathrm{~W} \mathrm{~m}^{-2}$ and considering a distance of $2800 \pm 200 \mathrm{pc}$, the central star should have a luminosity of $L(s)=(6.6 \pm 1) \times 10^{6} L_{\odot}$ equivalent to $M_{\text {bol }}(s)=-12^{m} .3 \pm 0^{m} .2$. The total flux corresponds with a star having a radius of $R \sim 96 R_{\odot}$ if the temperature $T_{\text {eff }} \sim 30000 \mathrm{~K}$ (Section 8).

A study is made of the past and present variable light behaviour and photometric characteristics of $\eta$ Car. Probably the star is already active since at least $1000 \mathrm{yr}$ ago. From the comparison of the colours of $\eta$ Car in different stages of its variation in the 19th century with bright late type stars, we conclude that the intrinsic colour in the maximum of $\sim 1843$ was $(B-V)_{\mathrm{J}, 0} \sim 0^{m} .9$ and in the minimum of $\sim 1890$ $(B-V)_{\mathrm{J}, 0} \sim 0^{m} .65$. (Section 9.1).

The sudden rise from 1930 to 1940 by $\sim 1^{m} .5$ photographically and the bluing by $\sim 0^{m} .6$ is likely caused by a decrease of dust close around the star, giving rise to a decrease of extinction (Section 9.2).

Around 1843 the object was presumably bolometrically at least $\sim 2^{m} .5$ brighter than at present, viz. $M_{\text {bol }} \leqslant-14$. 8 . At constant radius the central star should have had a temperature of $T_{\text {eff }} \sim 50000 \mathrm{~K}$ instead of the present value of $\sim 30000 \mathrm{~K}$ (Section 9.2).

Figure 24 summarizes the adopted temperature of the central star and its estimated mass loss rate during the three different stages. The hypothetical S Dor phases are schematically indicated by dotted curves. In the minima, the mass loss rates are supposed to be normal (similar to other S Dor type stars), so that the central star could 


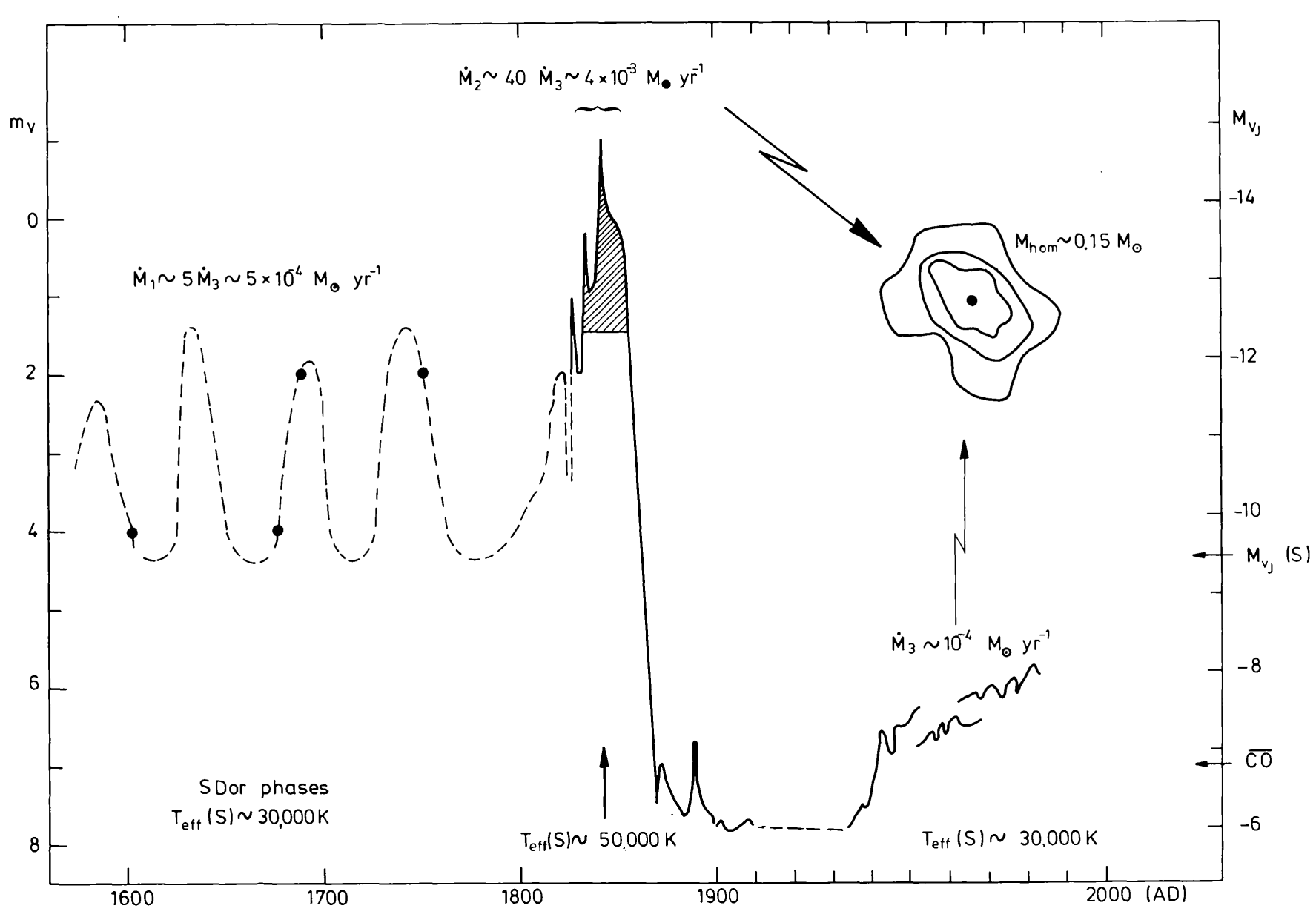

Fig. 24. A summary of the adopted mass loss rates and temperatures during the various stages of $\eta$ Car. The postulated S Dor phases occurring before 1800 , are schematically indicated by the dotted curves. The period of the luminosity excess is hatched. The kinematical age of the homunculus suggests that it has been mainly formed during this interval. (The homunculus is sketched at the right top by means of a few isophotes.) The absolute visual magnitude of the central star $(s)$ and that of the obscured central object $(\overline{c o})$ at present, are indicated at the right side.

be seen undisturbed. Therefore the absolute visual magnitude would not have been much different from the present one $\left(M_{V_{\mathrm{J}}} \sim-9^{m} .5\right)$, adopting that the intrinsic characteristics of the star did not change much within a few centuries. The corresponding apparent visual magnitude then would have been $m_{v} \sim 4.5$. In the maxima the mass loss rate is estimated to amount to 5 times the normal rate (that is thus when the star is in minimum state).

The excess of luminosity could be explained in terms of envelope-energized pulsations when the star was close to its Eddington limit (Sections 9.3 and 9.5). The amount of mass loss during the climax in 1843 is estimated to amount to $\dot{M} \sim 4 \times 10^{-3} M_{\odot} \mathrm{yr}^{-1}$. The total mass of the homunculus is probably $M_{\text {hom }} \sim 0.15 M_{\odot}$ and is presumably formed mainly during that climax. The mass loss rate just mentioned is 20 and 5 times lower than those estimated by Andriesse et al. (1978) and Hyland et al. (1979), respectively, and is probably more in accord with recent considerations on the mass loss of 
super- and hypergiants lying close to the limit of stellar existence (discussed by de Jager, 1984). The present mass loss rate is presumably $\dot{M} \sim 10^{-4} M_{\odot} \mathrm{yr}^{-1}$ (Section 9.3).

It is suggested that also P Cyg suffered from S Dor type outbursts in the 17 th century, but at a smaller scale than $\eta$ Car (Section 9.4).

It is not yet clear whether all supergiants go through the S Dor stage (which should be very short, in view of the low number of S Dor type stars), or whether it is confined to a small number of them because of special circumstances. A discussion is presented on the usually low expansion velocities of the envelopes of $\mathrm{S}$ Dor type stars (Section 9.5).

A study of the variations in the Balmer-jump suggests that most of the small amplitude light variations of $\eta$ Car during the last decade may be caused by temperature variations, of a few thousand degrees, of the central star. The Balmer-jump variation in 1981 however, suggests an additional cause namely that a new shell was expelled. This is supported by the spectroscopic study of Zanella et al. (1984). It is not unlikely that the star became slightly hotter since the last $30 \mathrm{yr}$. If this suspicion is true it could mean that the star, already in a post-Main Sequence stage (Section 9.4) is moving blueward in the HR diagram. According to Maeder (1983) a $120 M_{\odot}$ model can increase $\sim 1000 \mathrm{~K}$ within a decennium. For a normal star one would expect a decrease of the visual luminosity, while $\eta$ Car showed a steady rise during the last decennia. Perhaps the radius is also increasing. However, in such an intricate system, with degrading of energy into various components and a central star still able to expel new envelopes one may expect difficulties with the interpretation.

\section{Acknowledgements}

We are grateful to Prof. Dr H. J. G. L. M. Lamers for his help with respect to Section 9.3 and to Dr V. Icke for his aid and various suggestions concerning the mass loss problem. We also thank Prof. Dr J. M. Greenberg for his invaluable help concerning Sections 6 and 7, and Dr F. P. Israel for discussions with respect to Section 8.

We like to express our gratitude to Profs. C. de Jager, H. J. G. L. M. Lamers and J. M. Greenberg for their comments on the manuscript and to a number of persons who made additional observations. They are: P. Alphenaar, R. Bakker, J. Brand, E. J. de Geus, G. M. van Heerde, L. de Lange, Th. J. van der Linden, J. Lub, J. J. M. Meys, J. Oppe, R. F. A. Staller, M. J. J. Wiertz, and H. van Wimersma Greidanus and finally to Dr K. Davidson for his kindness to make available to us the computerized image of $\eta$ Car (Figure 12).

Part of the reduction has been made by computerprograms written by Dr J. Tinbergen, J. J. Schafgans, Dr J. Lub, and K. Weerstra.

Note added in proof. It is of interest to add the following relevant references. With respect to the temperature of the central star we should mention Andriesse et al. (1981) (Astron. Astrophys. 95, 202) who prefer the low temperature of $\sim 7500 \mathrm{~K}$. With the application of Andriesse's fluctuation theory they claim to have found an explanation 
for the blow-off phenomenon of $\eta$ Car (revealing $\dot{M} \sim 10^{-2} M_{\odot} \mathrm{yr}^{-1}$, similar to the value quoted in Section 9.3), the occurrence of single WR stars and the absence of massive red giants. Also Viotti et al. 1984 (preprint) favour such a low temperature for the central star.

Percy and Welch (1983) (Publ. Astron. Soc. Pacific 95, 491) have observed P Cyg on a number of nights in 1982 and found for the photometric variations a time scale of 30 to 50 days and an amplitude of $\sim 0^{m} .15$.

\section{References}

Abbott, D. C., Bieging, J. H., Churchwell, E., and Cassinelli, J. P.: 1980, Astrophys. J. 238, 196.

Abbott, D. C., Bieging, J. H., and Churchwell, E.: 1981, Astrophys. J. 250, 645.

Aitken, D. K. and Jones, B.: 1975, Monthly Notices Roy. Astron. Soc. 172, 141.

Aller, L. H.: 1970, Sky Telesc. 39, 368.

Allen, C. W.: 1955, Astrophysical Quantities, The Athlone Press, London.

Allen, C. W.: 1973, Astrophysical Quantities, 3rd edition, The Athlone Press, London.

Allen, D. A. and Glass, I. S.: 1976, Astrophys. J. 210, 666.

Andriesse, C. D., Donn, B. D., and Viotti, R.: 1978, Monthly Notices Roy. Astron. Soc. 185, 771.

Barlow, M. J. and Cohen, M.: 1977, Astrophys. J. 213, 737.

Bateson, F. M.: 1952-1970, Astron. Soc. New Zealand, Var. Star Section Circ.

Bessell, M. S.: 1979, Publ. Astron. Soc. Pacific 91, 589.

Burki, G., Heck, A., Bianchi, L., and Cassatella, A.: 1982, Astron. Astrophys. 107, 205.

Cannon, A. J.: 1897, Harvard Coll. Obs. Ann. 28, 175.

Cannon, A. J.: 1916, Harvard Coll. Obs. Ann. 76, 36.

Cassatella, A., Giangrande, A., and Viotti, R.: 1979, Astron. Astrophys. 71, L9.

Chini, R. and Krügel, E.: 1983, Astron. Astrophys. 117, 289.

Chlebowski, T., Seward, F. D., Swank, J., and Szymkowiak, A.: 1984, Astrophys. J. 281, 665.

Chlewicki, G., Greenberg, J. M., Aiello, S., Barsella, B., Patriarchi, P., and Perionotto, M.: 1984, Proceedings of the IVth European IUE Conference, ESA-SP-218, p. 507.

Craine, E. R.: 1974, Astrophys. J. 191, 105.

Davidson, K.: 1971, Monthly Notices Roy. Astron. Soc. 154, 415.

Davidson, K., Walborn, N. R., and Gull, T. R.: 1982, Astrophys. J. 254, L47.

Davidson, K., Dufour, R. J., Walborn, N. R., and Gull, T. R.: 1984, IAU Symp. 105, 261.

de Groot, M.: 1969, Bull. Astron. Inst. Neth. 20, 225.

de Jager, C.: 1980, The Brightest Stars, D. Reidel Publ. Co., Dordrecht, Holland.

de Jager, C.: 1981, S. D’Odorico, D. Baade, and K. Kjär (eds.) The Most Massive Stars, ESO Workshop, p. 67.

de Jager, C.: 1984, Astron. Astrophys. 138, 246.

de Vaucouleurs, G. and Eggen, O. J.: 1952, Publ. Astron. Soc. Pacific 64, 185.

Feinstein, A. and Marraco, H. G.: 1974, Astron. Astrophys. 30, 271.

Feinstein, A., Marraco, H. G., and Muzzio, J. C.: 1973, Astron. Astrophys. Suppl. 12, 331.

Gaviola, E.: 1950, Astrophys. J. 111, 408.

Gehrz, R. D. and Ney, E. P.: 1972, Sky Telesc. 44, 4.

Gehrz, R. D., Ney, E. P., Becklin, E. E., and Neugebauer, G.: 1973, Astrophys. Letters 13, 89.

Glass, I. S.: 1974, Monthly Notices Roy. Astron. Soc. 168, 249.

Glass, I. S.: 1984, Monthly Notices Roy. Astron. Soc. 209, 759.

Gratton, L.: 1963, Star Evolution, Academic Press, New York and London, p. 297.

Greenberg, J. M.: 1966, in B. Middlehurst and L. Aller (eds.), Nebulae and Interstellar Matter, Univ. of Chicago Press, p. 221.

Greve, A., van Genderen, A. M., Dennefeld, M., and Danziger, I. J.: 1982, Astron. Astrophys. 111, 171.

Groeneveld, I.: 1944, Veröff. Landessternwarte Heidelberg 14, No. 5, 1.

Harvey, P. M., Hoffmann, W. F., and Campbell, M. F.: 1978, Astron. Astrophys. 70, 165.

Herbst, W.: 1976, Astrophys. J. 208, 923. 
Humphreys, R. M.: 1978, Astrophys. J. Suppl. 38, 309.

Humphreys, R. M., Blaha, C., D’Odorico, S., Gull, T. R., and Benvenuti, P.: 1984, Astrophys. J. 278, 124.

Hyland, A. R., Robinson, G., Mitchell, R. M., Thomas, J. A., and Becklin, E. E.: 1979, Astrophys. J. 233, 145.

Icke, V.: 1981, Astrophys. J. 247, 152.

Innes, R. T. A.: 1903, Cape Ann. 9, 75B.

Johnson, H. L.: 1966, Ann. Rev. Astron. Astrophys. 4, 193.

Johnson, H. L.: 1968, 'Interstellar Extinction', in B. M. Middlehurst and L. H. Aller (eds.), Nebulae and Interstellar Matter, Univ. of Chicago Press.

Joyce, R. R.: 1975, Publ. Astron. Soc. Pacific 87, 917.

Koornneef, J.: 1978, Monthly Notices Roy. Astron. Soc. 184, 477.

Koornneef, J.: 1983, Astron. Astrophys. 128, 84.

Kurucz, R. L.: 1979, Astrophys. J. Suppl. 40, 1.

Lamers, H. J. G. L. M.: 1981, Astrophys. J. 245, 593.

Lamers, H. J. G. L. M., de Groot, M., and Cassatella, A.: 1983, Astron. Astrophys. 123, L8.

Lamers, H. J. G. L. M., Waters, L. B. F. M., and Wesselius, P. R.: 1984a, Astron. Astrophys. 134, L17.

Lamers, H. J. G. L. M., Korevaar, P., and Cassatella, A.: 1984b, Fourth European IUE Conference, ESA SP-218, p. 315.

Lub, J.: 1980, Internal Report, Leiden Observatory.

Lub, J. and Pel, J. W.: 1977, Astron. Astrophys. 54, 137.

Lub, J., van Paradijs, J. A., Pel, J. W., and Wesselius, P. R.: 1978, Astron. Astrophys. 72, 83.

Maeder, A.: 1980, Astron. Astrophys. 92, 101.

Maeder, A.: 1981a, Astron. Astrophys. 99, 97.

Maeder, A.: 1981b, Astron. Astrophys. 102, 401.

Maeder, A.: 1983, Astron. Astrophys. 120, 113.

Meaburn, J., Walsh, J. R., Hebden, J. C., Morgan, B. L., and Vine, H.: 1983, Monthly Notices Roy. Astron. Soc. 204, 418.

Melnick, J., Rinz, M. T., and Maza, J.: 1982, Astron. Astrophys. 111, 375.

Mitchell, R. M. and Robinson, G.: 1978, Astrophys. J. 220, 841.

Neckel, T. and Staude, H. J.: 1984, Astron. Astrophys. 131, 200.

Neugebauer, G. and Westphal, J. A.: 1968, Astrophys. J. 152, L89.

O’Connell, S. J., D. J. K.: 1956, Vistas Astron. 2, 1165.

Olnon, F. M.: 1984, Private Communication.

Pel, J. W.: 1976, Astron. Astrophys. Suppl. 24, 413.

Pel, J. W.: 1980, Internal Report, Leiden Observatory.

Pottasch, S. R., Wesselius, P. R., and van Duinen, R. J.: 1976, Astron. Astrophys. 47, 443.

Retallack, D. S.: 1983, Monthly Notices Roy. Astron. Soc. 204, 669.

Ringuelet, A. E.: 1958, Z. Astrophys. 46, 276.

Robinson, G., Hyland, A. R., and Thomas, J. A.: 1973, Monthly Notices Roy. Astron. Soc. 161, 281.

Rodgers, A. W. and Searle, L.: 1967, Monthly Notices Roy. Astron. Soc. 135, 99.

Rijf, R., Tinbergen, J., and Walraven, Th.: 1969, Bull. Astron. Inst. Neth. 20, 279.

Savage, B. D. and Mathis, J. S.: 1979, Ann. Rev. Astron. Astrophys. 17, 73.

Schmidt, G. D., Angel, J. R. P., and Beaver, E. A.: 1978, Astrophys. J. 219, 477.

Schwarzschild, M.: 1965, Structure and Evolution of Stars, Dover Publications, Inc., New York, p. 91.

Serkowski, K., Mathewson, D. S., and Ford, V. L.: 1975, Astrophys. J. 196, 261.

Sher, D.: (1965, Monthly Notices Roy. Astron. Soc. 129, 237.

Sparks, W. M., Starrfield, S., and Truran, J. W.: 1978, Astrophys. J. 220, 1063.

Stahl, O. and Wolf, B.: 1982, Astron. Astrophys. 110, 272.

Stahl, O., Wolf, B., Zickgraf, F. J., Bastian, U., de Groot, M. J. H., and Leitherer, C.: 1983a, Astron. Astrophys. 120, 287.

Stahl, O., Wolf, B., Klare, G., Cassatella, A., Krautter, J., Persi, P., and Ferrari-Toniolo, M.: 1983b, Astron. Astrophys. 127, 49.

Sterken, C.: 1977, Astron. Astrophys. 57, 361.

Stothers, R. and Chin, C.-W.: Astrophys. J. 264, 583.

Strecker, D. W., Erickson, E. F., and Witteborn, F. C.: 1979, Astrophys. J. Suppl. 40, 501.

Sutton, E., Becklin, E. E., and Neugebauer, G.: 1974, Astrophys. J. 190, L69. 
Thackeray, A. D.: 1953, Monthly Notices Roy. Astron. Soc. 113, 237.

Thackeray, A. D.: 1956, Observatory 76, 154.

Thackeray, A. D.: 1961, Observatory 81, 99.

Thackeray, A. D.: 1974, Monthly Notices Roy. Astron. Soc. 168, 221.

Thé, P. S. and Groot, M.: 1983, Astron. Astrophys. 125, 75.

Thé, P. S., Bakker, R., and Antalova, A.: 1980a, Astron. Astrophys. Suppl. 41, 93.

Thé, P. S., Bakker, R., and Tjin A Djie, H. R. E.: 1980b, Astron. Astrophys. 89, 209.

Tutukov, A. V.: 1981, in D. Sugimoto, D. Q. Lamb, and D. N. Schramm (eds.), 'Fundamental Problems in the Theory of Stellar Evolution', IAU Symp. 93, 137.

Van Blerkom, D.: 1978, Astrophys. J. 221, 186.

van den Bos, W.: 1938, Union Obs. Circ., No. 100, 522.

van Genderen, A. M.: 1979, Astron. Astrophys. Suppl. 38, 381.

van Genderen, A. M.: 1982, Astron. Astrophys. 112, 61.

van Genderen, A. M., Bijleveld, W., and van Groningen, E.: 1984, Astron. Astrophys. Suppl Ser. 58, 537.

van Genderen, A. M., Groot, M., and Thé, P. S.: 1983, Astron. Astrophys. 117, 53.

Viotti, R.: 1969, Astrophys. Space Sci. 5, 323.

Viotti, R. and Andriesse, C. D.: 1981, in S. D’Odorico, D. Baade, and K. Kjär (eds.), The Most Massive Stars, ESO Workshop, p. 141.

Visvanathan, N.: 1967, Monthly Notices Roy. Astron. Soc. 135, 275.

Walborn, N. R. and Gull, T. R.: 1982, Sky Telesc. 64, 16.

Walborn, N. R. and Hesser, J. E.: 1975, Astrophys. J. 199, 535.

Walborn, N. R. and Liller, M. H.: 1977, Astrophys. J. 211, 181.

Walborn, N. R., Blanco, B. M., and Thackeray, A. D.: 1978, Astrophys. J. 219, 498.

Walraven, Th. and Walraven, J. H.: 1960, Bull. Astron. Inst. Neth. 15, 67.

Warren-Smith, R. F., Scarrott, S. M., Murdin, P., and Bingham, R. G.: 1979, Monthly Notices Roy. Astron. Soc. 187, 761.

Wendker, H. J.: 1982, Astron. Astrophys. 116, L5.

Wesselius, P. R., van Duinen, R. J., de Jonge, A. R. W., Aalders, J. W. G., Luinge, W., and Wildeman, K. J.: 1982, Astron. Astrophys. Suppl. 49, 427.

Westphal, J. A. and Neugebauer, G.: 1969, Axtrophys. J. 156, L45.

White, R. L. and Becker, R. H.: 1983, Astrophys. J. 262, 657.

Whitelock, P. A., Feast, M. W., Carter, B. S., Roberts, G., and Glass, I. S.: 1983, Monthly Notices Roy. Astron. Soc. 203, 385.

Whitney, C. A.: 1952, Harvard Bull. 921, 8.

Wolf, B., Appenzeller, I., and Cassatella, A.: 1980, Astron. Astrophys. 88, 15.

Wolf, B., Appenzeller, I., and Stahl, O.: 1981, Astron. Astrophys. 103, 94.

Zanella, R., Wolf, B., and Stahl, O.: 1984, Astron. Astrophys. 137, 79.

Zinner, E.: 1952, Veröff. Remeis Sternw., No. 7, 1. 